University of Tennessee Health Science Center

UTHSC Digital Commons

$12-2006$

\title{
Depression, Anxiety, Hazardous Drinking, Subjective Burden, and Rewards in Family Caregivers of Patients With Chronic Liver Disease
}

Lois Vennesta Bolden

University of Tennessee Health Science Center

Follow this and additional works at: https://dc.uthsc.edu/dissertations

Part of the Diseases Commons, Nursing Commons, Other Mental and Social Health Commons, and the Substance Abuse and Addiction Commons

\section{Recommended Citation}

Bolden, Lois Vennesta, "Depression, Anxiety, Hazardous Drinking, Subjective Burden, and Rewards in Family Caregivers of Patients With Chronic Liver Disease" (2006). Theses and Dissertations (ETD). Paper 26. http://dx.doi.org/10.21007/etd.cghs.2006.0030.

This Dissertation is brought to you for free and open access by the College of Graduate Health Sciences at UTHSC Digital Commons. It has been accepted for inclusion in Theses and Dissertations (ETD) by an authorized administrator of UTHSC Digital Commons. For more information, please contact jwelch30@uthsc.edu. 


\title{
Depression, Anxiety, Hazardous Drinking, Subjective Burden, and Rewards in Family Caregivers of Patients With Chronic Liver Disease
}

\begin{abstract}
This descriptive correlation study had three purposes. The study first described depressive and anxiety symptom levels, prevalence of hazardous drinking, rewards, and subjective burden reported by family caregivers of patients with chronic liver disease (CLD). Second, the investigator compared depressive and anxiety symptom levels, prevalence of hazardous drinking, rewards, and subjective burden reported by African Americans family caregivers with those reported by Caucasian family caregivers of patients with CLD. The third purpose was to determine the predictors of subjective burden and mental health status of family caregivers of persons with CLD.
\end{abstract}

A convenience sample of 73 caregivers of patients receiving care in a university-based, hepatology practice located in a large metropolitan city in the Mid-South provided data for the study. Depressive symptoms were measured with the Center for Epidemiological Studies Depression Scale (CES-D). Anxiety was measured with the Hamilton Anxiety Rating Scale (HAM-A). Hazardous drinking was measured with Alcohol Use Disorders Identification Test (AUDIT). Subjective burden was measured using Zarit's Burden Interview (BI) and caregiver rewards were assessed with Picot Caregiver Rewards Scale (PCRS). An investigator developed demographic data form was used to obtain caregiver and patient characteristics. The Pearlin Stress Process Model (SPM) provided the conceptual basis for the study.

Cronbach alpha coefficients obtained for the CES-D, HAM-A, AUDIT, BI, and PCRS scales ranged from .72 to .93. Data were analyzed using descriptive statistics, independent sample $t$-tests, Mann Whitney tests, Pearson's product-moment correlation, Spearman's rho correlation, and stepwise multiple regression analyses. The sample consisted of more Caucasians (65.8\%) than African Americans (30.1\%) and more females $(78 \%)$ than males (22\%). Caregivers were typically married (53\%), middle-aged ( $48.2 \pm 14.7$ years) high school graduates $(12.4 \pm 2.6$ years). Most caregivers were employed full-time $(41.1 \%)$. The study sample reported mild depressive symptoms, little or no anxiety or hazardous drinking, mild distress for subjective burden, and moderate caregiver rewards scores. Caucasians had a slightly higher though nonsignificant depressive symptom score when compared to African Americans. Both African American and Caucasian caregivers in this study reported experiencing little to no anxiety and the prevalence of hazardous drinking was low for both groups. African Americans' mean subjective burden score reflected little to no burden while Caucasian caregivers' scores reflected mild burden; these differences were not statistically significant. African American caregivers reported significantly higher mean rewards scores compared to Caucasian caregivers.

Stepwise regression analyses were performed to determine the degree to which selected independent variables significantly contributed to the explained variance in depressive and anxiety symptom levels, subjective burden, and caregiver rewards for the entire sample. Income decrease, worried about children, caregiver ethnicity, and caregiver gender were regressed on depressive symptoms. The variable worried about children (beta $=.24, p=.05$ ) remained in the final model and accounted for $6 \%$ of the adjusted variance in depressive symptom scores $(F(1,62)=4.768, p=.03)$. Worried about children was significantly and positively associated with increased depressive symptoms. Caregiver support, income decrease, worried about children, and activities of daily living were regressed on anxiety symptom levels. None of these variables significantly predicted anxiety symptom levels. In contrast, income decrease (beta=.35, $\mathrm{p}=.003$ ) and worried about children (beta $=.35, \mathrm{p}=.003$ ) predicted $25 \%$ of the adjusted variance in subjective burden $(F(2,56)=10.9, p=\leq .000)$. Caregiver ethnicity (beta=.37, $p=.001)$ and employment (beta=-.33, $p=.003$ accounted for $22 \%$ of the variance in caregivers rewards $(F(2,66)=10.7, p=\leq .000)$. Caregivers who were African American reported greater rewards and unemployed caregivers reported fewer rewards associated with the caregiver role. Findings are consistent with previous studies showing 
that African American caregivers experience more caregiver rewards compared to Caucasian caregivers though differences in burden did not occur. Caregivers who were worried about their children experienced more depressive symptoms and subjective burden. In contrast with published studies in other caregiver populations, neither length of care nor severity of symptoms significantly correlated with subjective burden or depressive symptoms in the study sample. Programs have been developed in three states to off-set caregiving-related income decreases, which could be an effective approach in this sample of caregivers who reported significant decreases in income. Further research could be conducted using focus groups to explore caregiver concerns related to children and this information could provide direction to reduce the burden and depressive symptoms associated with this variable. Research is also needed to determine if ethnic differences in the perception of rewards mediate or moderate the experience of burden and depressive symptoms in the study population. Interventions are particularly needed for depressive symptoms as clinically significant levels were present and clinical referrals warranted in this study sample.

\section{Document Type}

Dissertation

Degree Name

Doctor of Philosophy (PhD)

\section{Program}

Nursing

\section{Research Advisor}

Mona Newsome Wicks, Ph.D., RN

\section{Keywords}

Caregiving, Burden, Caregiver, Chronic Liver Disease, Depressive symptoms

\section{Subject Categories}

Diseases | Medicine and Health Sciences | Nursing | Other Mental and Social Health | Substance Abuse and Addiction 
DEPRESSION, ANXIETY, HAZARDOUS DRINKING, SUBJECTIVE BURDEN, AND REWARDS IN FAMILY CAREGIVERS OF PATIENTS WITH CHRONIC

LIVER DISEASE

\author{
A Dissertation \\ Presented for the Graduate Studies Council \\ The University of Tennessee \\ Health Science Center \\ In Partial Fulfillment \\ Of the Requirements for the Degree \\ Doctor of Philosophy \\ From The University of Tennessee
}

By

Lois V. Bolden

December 2006 
Copyright (C) Lois V. Bolden, 2006 All rights reserved 


\section{DEDICATION}

This dissertation is dedicated to the memory of my mother,

Mrs. Mattie P. Bolden, who instilled in me faith, courage, and perseverance. 


\section{ACKNOWLEDGMENTS}

I would like to thank my dissertation chair, Dr. Mona N. Wicks, for her assistance in helping me reach this professional milestone. Additionally, I would like to express my sincere gratitude to the other members of my dissertation committee: Drs. Kelly Askins, Sarah Mynatt, Muriel Rice, Caroline Riely and Rebecca Winsett for their wonderful support and guidance through this milestone. The support and mentoring I received from my committee members has been invaluable. I also would like to extend my sincere gratitude to my brothers, sisters, nieces, nephews, friends and colleagues for their understanding and continuous encouragement.

Funding for this research was provided by a fellowship from the Ethnic Minority Fellowship from Substance Abuse Mental Health Services Administration (SAMHSA) and a grant from the Beta Theta Chapter-at-Large of Sigma Theta Tau. The Gerontological Society of America via the Copyright Clearance Center granted permission for reprint for Figure 1. 


\begin{abstract}
This descriptive correlation study had three purposes. The study first described depressive and anxiety symptom levels, prevalence of hazardous drinking, rewards, and subjective burden reported by family caregivers of patients with chronic liver disease (CLD). Second, the investigator compared depressive and anxiety symptom levels, prevalence of hazardous drinking, rewards, and subjective burden reported by African Americans family caregivers with those reported by Caucasian family caregivers of patients with CLD. The third purpose was to determine the predictors of subjective burden and mental health status of family caregivers of persons with CLD.
\end{abstract}

A convenience sample of 73 caregivers of patients receiving care in a universitybased, hepatology practice located in a large metropolitan city in the Mid-South provided data for the study. Depressive symptoms were measured with the Center for Epidemiological Studies Depression Scale (CES-D). Anxiety was measured with the Hamilton Anxiety Rating Scale (HAM-A). Hazardous drinking was measured with Alcohol Use Disorders Identification Test (AUDIT). Subjective burden was measured using Zarit's Burden Interview (BI) and caregiver rewards were assessed with Picot Caregiver Rewards Scale (PCRS). An investigator developed demographic data form was used to obtain caregiver and patient characteristics. The Pearlin Stress Process Model (SPM) provided the conceptual basis for the study.

Cronbach alpha coefficients obtained for the CES-D, HAM-A, AUDIT, BI, and PCRS scales ranged from .72 to .93 . Data were analyzed using descriptive statistics, independent sample $t$-tests, Mann Whitney tests, Pearson's product-moment correlation, 
Spearman's rho correlation, and stepwise multiple regression analyses. The sample consisted of more Caucasians (65.8\%) than African Americans (30.1\%) and more females (78\%) than males (22\%). Caregivers were typically married (53\%), middle-aged (48.2 \pm 14.7 years) high school graduates $(12.4 \pm 2.6$ years). Most caregivers were employed full-time (41.1\%).The study sample reported mild depressive symptoms, little or no anxiety or hazardous drinking, mild distress for subjective burden, and moderate caregiver rewards scores. Caucasians had a slightly higher though non-significant depressive symptom score when compared to African Americans. Both African American and Caucasian caregivers in this study reported experiencing little to no anxiety and the prevalence of hazardous drinking was low for both groups. African Americans' mean subjective burden score reflected little to no burden while Caucasian caregivers' scores reflected mild burden; these differences were not statistically significant. African American caregivers reported significantly higher mean rewards scores compared to Caucasian caregivers.

Stepwise regression analyses were performed to determine the degree to which selected independent variables significantly contributed to the explained variance in depressive and anxiety symptom levels, subjective burden, and caregiver rewards for the entire sample. Income decrease, worried about children, caregiver ethnicity, and caregiver gender were regressed on depressive symptoms. The variable worried about children $(\beta=.24, p=.05)$ remained in the final model and accounted for $6 \%$ of the adjusted variance in depressive symptom scores $(\mathrm{F}(1,62)=4.768, p=.03)$. Worried about children was significantly and positively associated with increased depressive symptoms. Caregiver support, income decrease, worried about children, and activities of daily living 
were regressed on anxiety symptom levels. None of these variables significantly predicted anxiety symptom levels. In contrast, income decrease $(\beta=.35, \mathrm{p}=.003)$ and worried about children $(\beta=.35, \mathrm{p}=.003)$ predicted $25 \%$ of the adjusted variance in subjective burden $(\mathrm{F}(2,56)=10.9, p=\leq .000)$. Caregiver ethnicity $(\mathrm{B}=.37, p=.001)$ and employment ( $\mathrm{B}=-.33, p=.003$ accounted for $22 \%$ of the variance in caregivers rewards ( $\mathrm{F}$ $(2,66)=10.7, p=\leq .000)$. Caregivers who were African American reported greater rewards and unemployed caregivers reported fewer rewards associated with the caregiver role. Findings are consistent with previous studies showing that African American caregivers experience more caregiver rewards compared to Caucasian caregivers though differences in burden did not occur. Caregivers who were worried about their children experienced more depressive symptoms and subjective burden. In contrast with published studies in other caregiver populations, neither length of care nor severity of symptoms significantly correlated with subjective burden or depressive symptoms in the study sample. Programs have been developed in three states to off-set caregiving-related income decreases, which could be an effective approach in this sample of caregivers who reported significant decreases in income. Further research could be conducted using focus groups to explore caregiver concerns related to children and this information could provide direction to reduce the burden and depressive symptoms associated with this variable. Research is also needed to determine if ethnic differences in the perception of rewards mediate or moderate the experience of burden and depressive symptoms in the study population. Interventions are particularly needed for depressive symptoms as clinically significant levels were present and clinical referrals warranted in this study sample. 


\section{TABLE OF CONTENTS}

CHAPTER 1. Introduction . . . . . . . 1

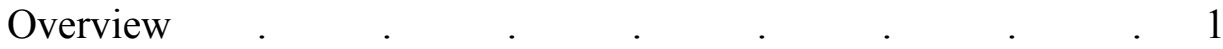

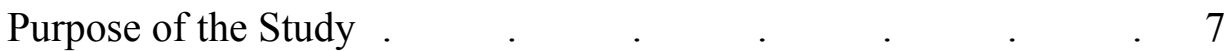

Aims and Research Questions $\quad . \quad$. $\quad . \quad$. $\quad .99$

Specific Aim One . $\quad . \quad$. $\quad . \quad$. $\quad .99$

Specific Aim Two . $\quad . \quad$. $\quad . \quad$. $\quad .10$

Specific Aim Three $\quad . \quad$. $\quad . \quad$. $\quad . \quad$. $\quad . \quad$. 10

Conceptual Framework . $\quad . \quad$. $\quad . \quad$. $\quad$. $\quad . \quad 10$

Background and Context Components $\quad$. $\quad$. 16

Primary and Secondary Stressors . . . . . . 17

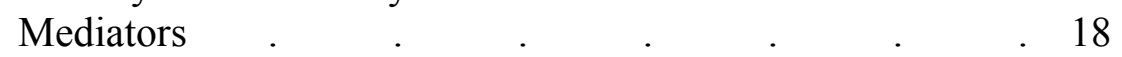

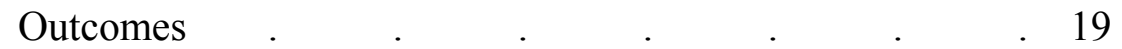

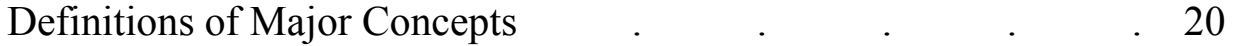

Depressive Symptoms _ . . . . . . 20

Anxiety . . . . . . . . . . $\quad$. 21

Hazardous Drinking . . . . . . . . 21

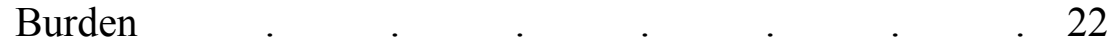

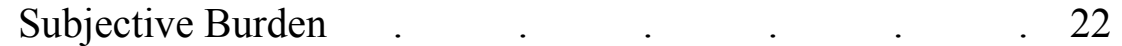

Caregiver Rewards . . . . . . . . . 23

Characteristics . . . . . . . . 23

Caregiver Characteristics . . . . . . 23

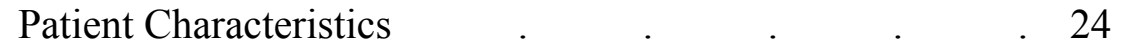

Chronic Liver Disease $\quad . \quad$. . . $\quad .25$

Concept Relationships . . . . . . . . . 25

Summary . . . . . . . . . . . . . .

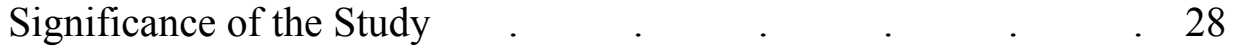

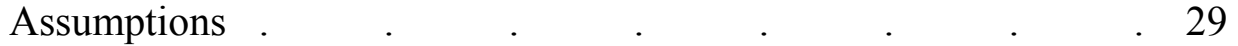

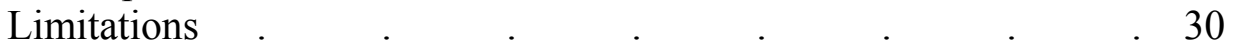

CHAPTER 2. Review of Literature. . . 32

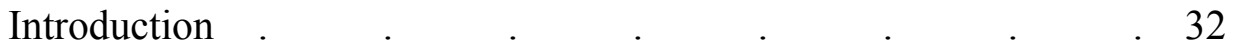

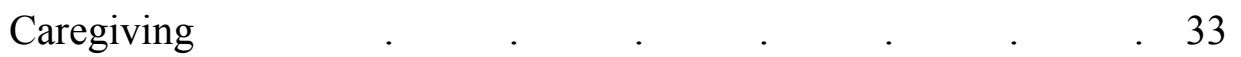

Caregiver Characteristics $\quad . \quad$. $\quad . \quad$. $\quad . \quad 34$

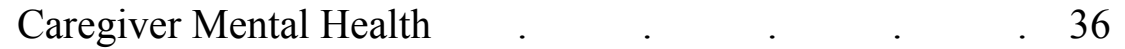

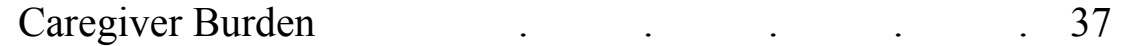

$\begin{array}{lllll}\text { Caregiver Rewards } \quad . \quad & \text {. } & \text {. } & 39\end{array}$

Caregiver Characteristics and Caregiver Burden . . 39

Caregiver Age and Caregiver Burden. $\quad$. . . . 40

Caregiver Ethnicity and Caregiver Burden . . . . . . 41 
Caregiver Gender and Caregiver Burden 42

Caregiver Relationship to Patient and Subjective

Caregiver Burden . . . . . . . . . . 43

Caregiver Socioeconomic Status and Caregiver Burden . 44

Caregiver Support and Caregiver Burden . . . . . 45

Caregiver Characteristics and Caregiver Depression,

Anxiety, and Substance Use . . . . . . . 46

Caregiver Characteristic and Caregiving Rewards . . . 48

Patient Characteristics and Caregiver Burden . . . 49

Patient Age and Caregiver Burden . . . . . $\quad .50$

Patients' Length of Illness and Caregiver Burden. . . . 51

Patients' Living Arrangements and Caregiver Burden. $\quad$. 52

Summary of the Review of Literature $\quad$. $\quad . \quad$. $\quad .53$

CHAPTER 3. Methodology . . . . . . . $\quad$. 55

Research Design $\quad . \quad$. $\quad . \quad$. $\quad . \quad$. $\quad . \quad 55$

Sample and Setting $\quad . \quad$. $\quad . \quad$. $\quad . \quad$. $\quad . \quad 55$

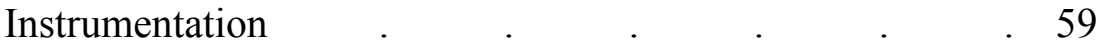

Family Caregiver Demographic Data Form . . 59

Center for Epidemiological Studies Depression Scale

(CES-D) . $\quad . \quad . \quad . \quad . \quad . \quad .60$

Hamilton Anxiety Rating Scale (HAM-A) 62

Alcohol Use Disorders Identification Test (AUDIT) 62

Burden Interview (BI) . . . . . . . . 64

Picot Caregiver Rewards Scale (PCRS) . . . 65

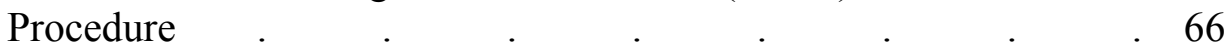

Site Preparation $\quad . \quad$. $\quad . \quad$. $\quad . \quad$. $\quad .67$

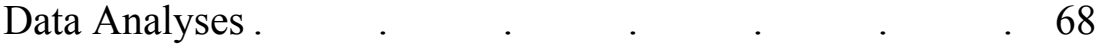

Analysis of Sample Characteristics ․ . . $\quad .68$

Specific Aim One . $\quad . \quad$. $\quad . \quad$. $\quad . \quad$. 69

Specific Aim Two. $\quad . \quad$. $\quad . \quad$. 69

Specific Aim Three . . . . . . $\quad .70$

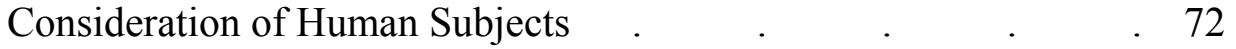

$\begin{array}{llllllll}\text { CHAPTER 4. } & \text { Results } \quad . \quad & . & . & & & & \end{array}$

Caregiver Characteristics . $\quad . \quad$. $\quad . \quad$. $\quad .76$

Care Recipients' Characteristics. $\quad$. $\quad$. $\quad$. $\quad$. 79

Mental Health, Burden, Reward Scores . . . . $\quad$. 79

Research Aims, Questions, and Findings. . . . . . $\quad .82$

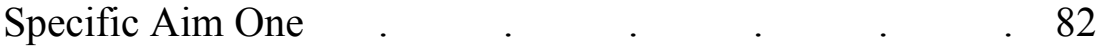

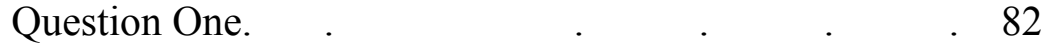

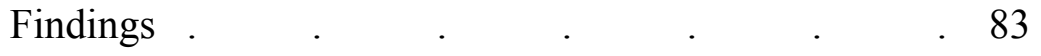




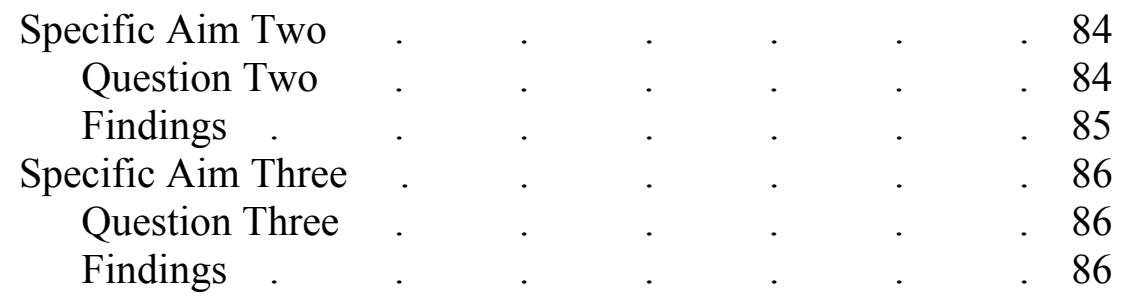

CHAPTER 5. $\quad$ Discussion and Implications. $\quad . \quad$. . 97

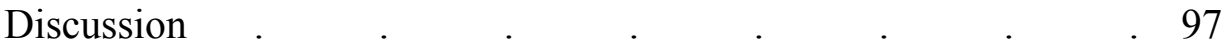

Demographic Findings. $\quad$. . . 997

Mental Health Symptoms and Hazardous Drinking . . 99

Anxiety Symptom Levels . . . . . . . 100

Subjective Burden and Caregiver Rewards . . . 102

Ethnic Differences in Subjective Burden and Caregiver

Rewards . . . . . . . . 104

Predictors of Mental Health and Burden . . . . 106

Limitations and Strengths . . . . . . . . . . 107

Practice and Policy Implications $\quad . \quad$. $\quad . \quad$. $\quad . \quad$. 110

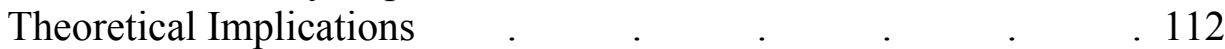

Recommendations for Further Research . . . . . . 113

Summary . . . . . . . . . . . . . .

LIST OF REFERENCES . . . . . . . . . . . 115

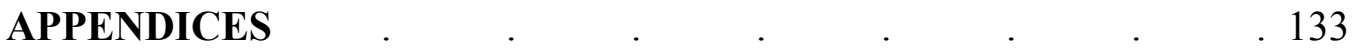

Appendix A. Child Pugh Score . $\quad$. $\quad$. $\quad$. $\quad .134$

Appendix B. Family Caregiver Demographic Data Form . . 136

Appendix C. Center for Epidemiological Studies Depression

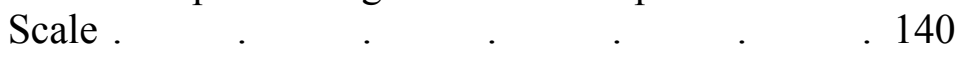

Appendix D. Hamilton Anxiety Rating Scale (HAM-A) . $\quad .143$

Appendix E. Alcohol Use Disorders Identification Test. . . . 146

Appendix F. Burden Interview . . . . . . . . 149

Appendix G. Picot Caregiver Rewards Scale . . . 153

Appendix H. Institutional Review Board Approval Letter . $\quad .158$

Appendix I. Caregiver Informed Consent Form . . 160

Appendix J. Patient Informed Consent Form . . 167

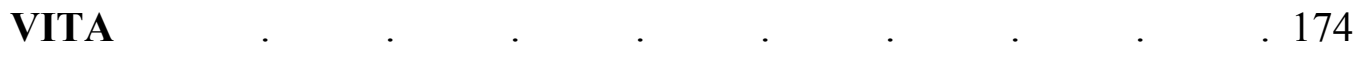




\section{LIST OF TABLES}

Table 1. Demographic Characteristics of Caregivers of Individuals with Chronic Liver Disease . . . . . . 77

Table 2. Characteristics of Care Recipients Diagnosed with Chronic

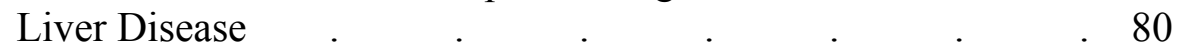

Table 3. Family Caregiver Scores on Mental Health, Burden, and Reward

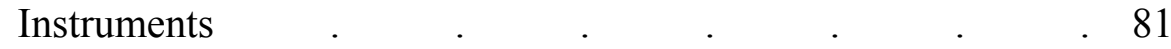

Table 4. Dummy Codes of Caregiver Demographic Variables . $\quad . \quad 88$

Table 5. Correlation Analysis between Caregiver Characteristics, Depressive and Anxiety Symptoms, Hazardous Drinking, and

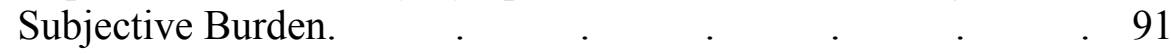

Table 6. Stepwise Multiple Regression Analysis of Depressive Symptoms and Caregiver Gender, Caregiver Ethnicity, Caregiver Employment, and Worried about Children. . 93

Table 7. Stepwise Multiple Regression Analysis of Subjective Burden and Caregiver Income Decrease, Worried about Children,

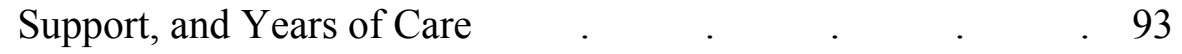

Table 8. Stepwise Multiple Regression Analysis of Caregiver Reward and Caregiver Ethnicity, Caregiver Relationship, Employment, and Marital Status. . $\quad . \quad$. $\quad . \quad$. 95

Table 9. Collinearity Diagnostics for the Model of Depressive Symptoms 95

Table 10. Collinearity Diagnostics for the Model of Anxiety Symptom Levels . . 95

Table 11. Collinearity Diagnostics for the Model of Subjective Burden Levels . 96

Table 12. Collinearity Diagnostics for the Model of Caregiver Rewards . 96 


\section{LIST OF FIGURES}

Figure 1. Caregiving and the Stress Process: An Overview of

Concepts and Their Measures. $\quad . \quad$. $\quad$. $\quad$. 12

Figure 2. Conceptual Model Depicting Relationships among

Primary and Secondary Stressors, Background and

Contextual Factors, Patient Characteristics, Mediators, and

Caregiver Stress Outcomes

14 


\section{CHAPTER 1. INTRODUCTION}

\section{Overview}

Cost-containment efforts by the insurance industry and federal government have led to an increase in family-based care of chronically ill individuals. Between 1990 and 1995, marked declines in hospital lengths of stay resulted in earlier discharge of more acutely ill patients (Sochalski \& Patrician, 1998). Further compounding the need for family-based home care is the increased number of individuals who survive illnesses that were previously fatal. Although many of these individuals survive the acute phase of these illnesses most often live with chronic, disabling conditions (Bodenheimer, Lorig, Holman, \& Grumbach, 2002; Roberts, 2000). A significant number of individuals cared for within the home have chronic liver disease (CLD). Of the 90 million Americans diagnosed with chronic illnesses an estimated 3 million are diagnosed with chronic liver disease (Habib, Bond, \& Heuman, 2001; Roberts, 2000). Disorders most often leading to CLD include alcoholic liver disease and hepatitis C. Approximate $15 \%$ to $20 \%$ of people who abuse alcohol develop alcoholic cirrhosis, and consequently alcoholic liver disease (Menon, Gores, \& Shah, 2001). Hepatitis $\mathrm{C}$ is characterized by fibrosis and an estimated $20 \%$ of patients develop progressive liver disease and cirrhosis, a major cause of CLD. Hepatitis C accounts for 12,000 deaths annually and is the most common cause of referral for liver transplantation (Clark, Mahoney, Clark, \& Eriksen, 2002; Koff, 1998; Moylan \& Muir, 2005; Patel, Muir, \& McHutchison, 2006). 
Changes in the healthcare system and health of many individuals have resulted in an increased need for family caregiving; however, providing care can be stressful for family caregivers. Stress related to caregiving can lead to burden that affects the caregiver and the care recipient. Chronic stress, in the presence of inadequate resources, places the health status of the caregiver and care recipient at risk for deterioration (Fortis Report, National Family Caregivers Association NFCA, 1998). Isolation, loss of productivity, depression, and stress are symptoms of caregiver burden (NFCA, 1998). Significant patient dependency and increasingly severe symptoms may contribute to physical and mental illness in caregivers (Pearlin, Mullan, Semple, \& Skaff, 1990). When caregiving requires a significant amount of the caregiver's time, there is less time for a career or social life leading to loss of productivity and isolation. Many caregivers (including caregivers of persons with CLD) experience declines in health status, particularly mental health status (NFCA, 1998; Polen \& Green, 2001).

Depression is particularly common in the presence of significant caregiver burden (NFCA, 1998). According to the National Institute of Health (NIH, 1998) and others (Kaneda \& Fujii, 2000), anxiety frequently coexists with depression and the symptoms sometimes overlap in individuals suffering from the disorders. This decline in caregiver mental health is especially important because caregivers may become unable to care for their chronically ill relative if significant burden and associated health impairments occur. The caregiver's inability to provide care for the ill relative has broad implications for the health and well-being of the patient, caregiver, and family unit. Researchers have neither studied caregiver burden nor its attributes in caregivers of persons diagnosed with CLD 
nor have they determined if depressive and anxiety symptoms are common in these caregivers.

Research studies link excessive drinking and alcohol abuse with depression (Dixit \& Crum, 2000; Holahan, Moos, Holahan, Cronkite, \& Randall, 2003; Schutte, Moos, \& Brennan, 1995); however, no researchers have specifically investigated hazardous drinking in caregiver populations. Since depression is significantly associated with caregiver burden (Chiriboga, Weiler, \& Nielsen, 1998; Haley et al., 1995; White, Townsend, \& Stephens, 2000) there is the possibility that alcohol misuse is associated with depression in this population as well. Researchers have also compared patterns of alcohol use by gender and in ethnic minority groups (Crum \& Anthony, 2000; Ross, Fortney, Lancaster, \& Booth, 1998). These studies show a significant relationship between increased depression and heavier alcohol consumption in women (Dixit \& Crum, 2000; Schutte et al., 1995). Additionally, studies show higher alcohol use in African American and Mexican American individuals when compared to other ethnic minority populations (Reardon \& Buka, 2002; Tam, Weisner, \& Mertens, 2000; Wells, Klap, Kioke, \& Sherbourne, 2001). However, investigators who reported differences in alcohol use across ethnic minority populations did not control for socioeconomic status, which tends to be lower in these populations (Wells et al., 2001). Alcohol abuse is more common in persons with low compared to high incomes (Van Oers, Bongers, Van De Goor, \& Garretsen, 1999). Caregivers are likely to share similar ineffective coping strategies with the general population, especially when they are significantly stressed or depressed. Obtaining information about the prevalence of excessive drinking in family caregivers of individuals diagnosed with CLD is imperative because hazardous drinking 
places family caregivers at risk for health problems and can disrupt the caregiving process. If CLD in the care recipient was caused by excessive use of alcohol, the caregiver is affected in another way. Researchers have shown that the prevalence of alcoholism and other disorders such as depression and anxiety were higher among those family members who grew up or resided with a family member who was a problem drinker. (Anda et al., 2002; Hurcom, Copello, \& Orford, 2000; Preuss, Schuckit, Smith, Barnow, \& Danko, 2002). Studies show that excessive alcohol use is one of the most common causes of CLD. Therefore, the possibility of the care recipient being involved in alcohol abuse is great. Caregivers of persons who have alcohol-associated CLD may share a similar increased prevalence of hazardous drinking if they reside with care recipients who abuse alcohol.

Patients with more advanced CLD manifest severe symptoms that frequently require the assistance of a caregiver. Debilitating fatigue, pruritis, ascites, anorexia, malnutrition, and encephalopathy are common (Habib et al., 2001). Because of the severity of the symptoms and the degree of functional impairment experienced by persons with CLD, caring for this population may be associated with significant caregiver burden. Caregivers of relatives diagnosed with hepatitis $\mathrm{C}$ may participate in preventive caregiving. Preventive caregiving refers to the time spent trying to maintain the physical and mental health of the patient to delay or prevent disease progression. Caregivers of persons with CLD may also be involved in supervisory caregiving, which is checking on the family member and arranging activities such as medical appointments (Ekwall, Sivberg, \& Hallberg, 2004). There is no research in the literature focusing on the emotional toll that caregivers of patients with hepatitis C or CLD in general encounter. 
Like caregivers assisting persons with other chronic diseases, some caregivers of patients with CLD probably experience significant caregiver burden.

Caregiver burden has been delineated as subjective and objective burden and is defined as the amount of time and energy required to provide care for another individual (Biegel, Sales, \& Schultz, 1991). Objective burden refers to the stress associated with tasks and activities related to providing care (Pearlin et al., 1990). Subjective burden is the burden perceived by caregivers (Montgomery, Gonyea, \& Hooyman ,1985). Subjective burden, the focus of this study, varies between caregivers because it reflects the awareness, affective orientation, and perceptions of the caregiver related to their caregiving experiences (Biegel et al., 1991). Caregiving and its consequences are also influenced by characteristics of the caregiver. Characteristics such as age, gender, ethnicity, education, and economic status influence the caregiver's perception of stress (Pearlin et al., 1990). Subjective caregiver burden and depression, for example, are more common in female caregivers (Belasco \& Sesso, 2002; Chiroboga, 1998; Gallicchio et al., 2002; Nagatomo et al., 1999).

Limited information exists in the literature comparing African American and Caucasian caregivers and their response to burden, although ethnicity is identified as a significant factor influencing the perception of burden in caregivers (Dilworth-Anderson et al., 2002; Pearlin et al., 1990). Moreover, findings from available studies are equivocal. For an example, Haley (1996) tested the Stress Process Model in a sample of African American and Caucasian caregivers of dementia patients finding that Caucasian caregivers reported higher depression scores on the Center for Epidemiological Studies Depression Scale (CES-D) than African American caregivers. In contrast, White et al. 
(2000), using the CES-D, found no difference in the depression scores reported by African American and Caucasian family caregivers. The discrepancies in the two studies may be attributed to the characteristics of the study. White et al. (2000) used a homogenous study with daughters and daughter-in-laws as caregivers of individuals with other diseases compared to Haley's et al. (1996) study sample included all family members as caregivers of individuals with dementia.

Health disparities and negative health outcomes are common among ethnic minorities, including caregivers. The Institute of Medicine Study: Unequal Treatment: Confronting Racial and Ethnic Disparities in Healthcare (IOM, 2001), documents significant racial and ethnic disparities in the United States with regard to accessing medical and mental health care services. Therefore, the caregiver's ethnicity not only influences perceptions of burden it also influences experience, opportunity, and choice in seeking healthcare (Pearlin et al., 1990). The caregiver's exposure to discrimination also affects patterns of seeking medical and mental health services consequently affecting health-related outcomes (Baker, \& Bell, 1999; Cain \& Kington, 2003; Williams \& Williams-Morris, 2000; Perlick, 2001; Flaskerud, Carter \& Lee, 2000).

Not all caregivers report burden, many caregivers report rewards associated with the role (Picot, 1995); however, the rewards of caregiving are understudied. Caregiving rewards include positive feelings, obligations, or gains felt because of providing care for family members (Picot, 1995). Caregivers who described the caregiving experience as rewarding, expressed joy and satisfaction associated with ensuring positive outcomes, meeting a valued obligation, and spending time with a loved one (Cohen, Colantonio, \& Venich, 2002; Sterrit \& Pokorny, 1998). Previous studies have shown that ethnicity 
plays a significant role in the caregiver's appraisal of the caregiving experience as it relates to rewards and positive experiences (Picot et al., 1997; White et al., 2000). In several studies, African American women reported higher levels of parent care rewards when compared to Caucasian women in the sample (Picot et al., 1997; White et al., 2000). Both Picot et al. (1997) and White et al. (2000) attributed cultural beliefs such as religiosity as a contributing factor in the perception of rewards reported by African American caregivers. These studies typically included small, convenience samples; consequently, more studies are needed to confirm or refute these findings in this understudied caregiver population.

\section{Purpose of the Study}

As a result of trends in healthcare, more individuals with chronic illnesses are receiving outpatient treatment for their illnesses (Bodenheimer et al.; 2002, Roberts, 2000). Patients are living longer with chronic illnesses and family caregivers are assisting ill family members in the home. Responsibilities such as caring for families of their own and participating in careers coupled with caregiving responsibilities can cause strain for caregivers. Strain associated with multiple roles may cause deteriorations in physical and mental health status and significant burden. Increasing burden related to caregiving can interfere with the caregiving role and affect the health of both the caregiver and care recipient. Many studies focus on family caregivers of persons diagnosed with cognitive impairments and other chronic illnesses (Acton, 2002; Bedard et al., 2001; Belasco et al., 2002; Burgner \& Twigg, 2002; Cain \& Wicks, 2000; Karlawish et al., 2001; Robinson, Adkisson, \& Weinrich, 2001; Teel , Duncan, \& Lai, 
2001). Although caregivers may share similar experiences across chronic illnesses, there are factors that are unique to the CLD population that likely create disease-specific challenges for these caregivers. Chronic liver disease is characterized by severe signs and symptoms such as fatigue, pruritis, ascites, anorexia, nutritional deficits, and cognitive changes related to encephalopathy (Habib, Bond, \& Heuman, 2001). Biegel (1991) describes the effects of severe symptoms on caregivers "as a stressor that is central to the amount of strain experienced" (p.201). The disabling symptoms associated with CLD probably contribute significant strain that increases the risk for depression and anxiety in this population of caregivers. Identifying caregivers at risk for depressive and anxiety symptoms can stimulate clinicians to provide information or resources to family caregivers to prevent negative health outcomes. Moreover, hepatitis $\mathrm{C}$ is the most common liver disease in the United States and often leads to cirrhosis of the liver (Koff, 1998; Patel, Muir, \& McHutchison, 2005). The disease-related morbidity and toll of providing care likely contributes to the concerns experienced by caregivers of persons with hepatitis C and persons with CLD in general. Distress related to these caregiving activities could occur. Thus, it is important to understand the factors that influence mental health status, the prevalence of hazardous drinking, subjective burden, and rewards for family caregivers of CLD patients. Despite the prevalence of the disease and severity of symptoms and their potential negative effects on caregivers, there are few studies examining the experiences of these caregivers. Information obtained from this study with regard to mental health status, subjective burden and caregiver rewards, reported by family caregivers of CLD patients will assist healthcare providers to develop appropriate 
interventions that are designed to maintain physical and mental health in this caregiver population.

\begin{abstract}
Aims and Research Questions
The Stress Process Model influenced the aims and research questions that guided this study. A detailed discussion of this model and its relevance for the current study is presented in the section that describes the conceptual framework. This study had three purposes. The study first described depressive and anxiety symptom levels, prevalence of hazardous drinking, rewards, and subjective burden reported by family caregivers of patients with CLD. Second, the investigator compared depressive and anxiety symptom levels, prevalence of hazardous drinking, rewards, and subjective burden reported by African Americans family caregivers of patients with CLD with those reported by Caucasians family caregivers of patients with CLD. The third purpose was to determine the predictors of subjective burden and mental and physical health status of family caregivers of persons with CLD. Three study aims and related research questions address the aforementioned purposes:
\end{abstract}

\title{
$\underline{\text { Specific Aim One }}$
}

Describe depressive symptom levels, anxiety symptom levels, and the prevalence of hazardous drinking, subjective burden, and caregiver rewards in family caregivers of persons with CLD. 
Related research question 1: What are the depressive and anxiety symptom levels and prevalence of hazardous drinking, subjective burden, caregiver rewards reported by family caregivers of patients with CLD?

\section{$\underline{\text { Specific Aim Two }}$}

Compare the depressive symptom levels, anxiety symptom levels, subjective burden, and caregiver rewards reported by African American and Caucasian family caregivers of persons with CLD.

Related research question 2: What are the differences in levels of depressive symptoms, anxiety symptoms, subjective burden and rewards reported by African American and Caucasian family caregivers of patients with CLD?

\section{Specific Aim Three}

Explicate the predictors of mental health status (depressive symptoms, anxiety levels), subjective burden, and caregiver rewards of family caregivers of persons with CLD.

Related research question 3: What are the predictors of mental health (depressive symptoms, anxiety levels), subjective burden, and caregiver rewards of family caregivers of patients with CLD?

\section{Conceptual Framework}

A conceptual framework provides structure for the phenomenon of interest and a clear picture of the relationship between the concepts (Fawcett, 2000). Concepts and 
terms that name and describe the phenomenon are the structural components of the conceptual framework (Burns \& Groves, 2001). In this study, Pearlin's Stress Process Model (SPM) (see Figure 1) facilitated the selection of the study variables (depression, anxiety, hazardous drinking, subjective burden, and caregiver rewards in caregivers of individuals diagnosed with CLD), instruments, and design. Pearlin and colleagues' (1990) SPM was originally used to describe the impact of stress in caregivers of patients with Alzheimer's disease. However, the model may be applicable to caregivers of individuals diagnosed with CLD and other chronic illnesses because many of the variables in the model (Figure 2) may have relevance regardless of the care recipient's diagnosis. Like persons with Alzheimer's disease, patients with CLD often experience significant cognitive impairment with disease progression. Several researchers used the SPM as a framework to study caregivers' of persons diagnosed with chronic illnesses such as cancer, end stage renal disease (ESRD), and cognitive impairment and caregivers of chronic obstructive pulmonary disease (COPD) (Cain \& Wicks, 2000; Haley et al., 2003; Harris, 2003; Wallsten, 2000). Providing care for chronically ill family members is a major stressor and synonymous with burden (Fredman et al., 1995; Zarit 1991). In the SPM, Pearlin and colleagues (1990) describe four domains: the background and context of stress, the stressors, mediators of stress, and the outcomes or manifestations of stress (Figure 1). Each domain will be discussed in the following paragraphs. 
Figure 1. Caregiving and the Stress Process: An Overview of Concepts and Their Measures.

Source. Pearlin, L., Mullan, J., Semple, S., \& Skaff, M. (1990). Caregiving and the stress process: An overview of concepts and their measures. The Gerontologist, 30(5), 583-594.

Harris, T. T. (2003). Burden and health in caregivers of persons with kidney disease. Unpublished doctoral dissertation, University of Tennessee, Memphis.

Copyright(C 1990 by The Gerontological Society of America. Reprinted with permission by The Gerontological Society of America via the Copyright Clearance Center and Dr. T. Harris-Beard. 


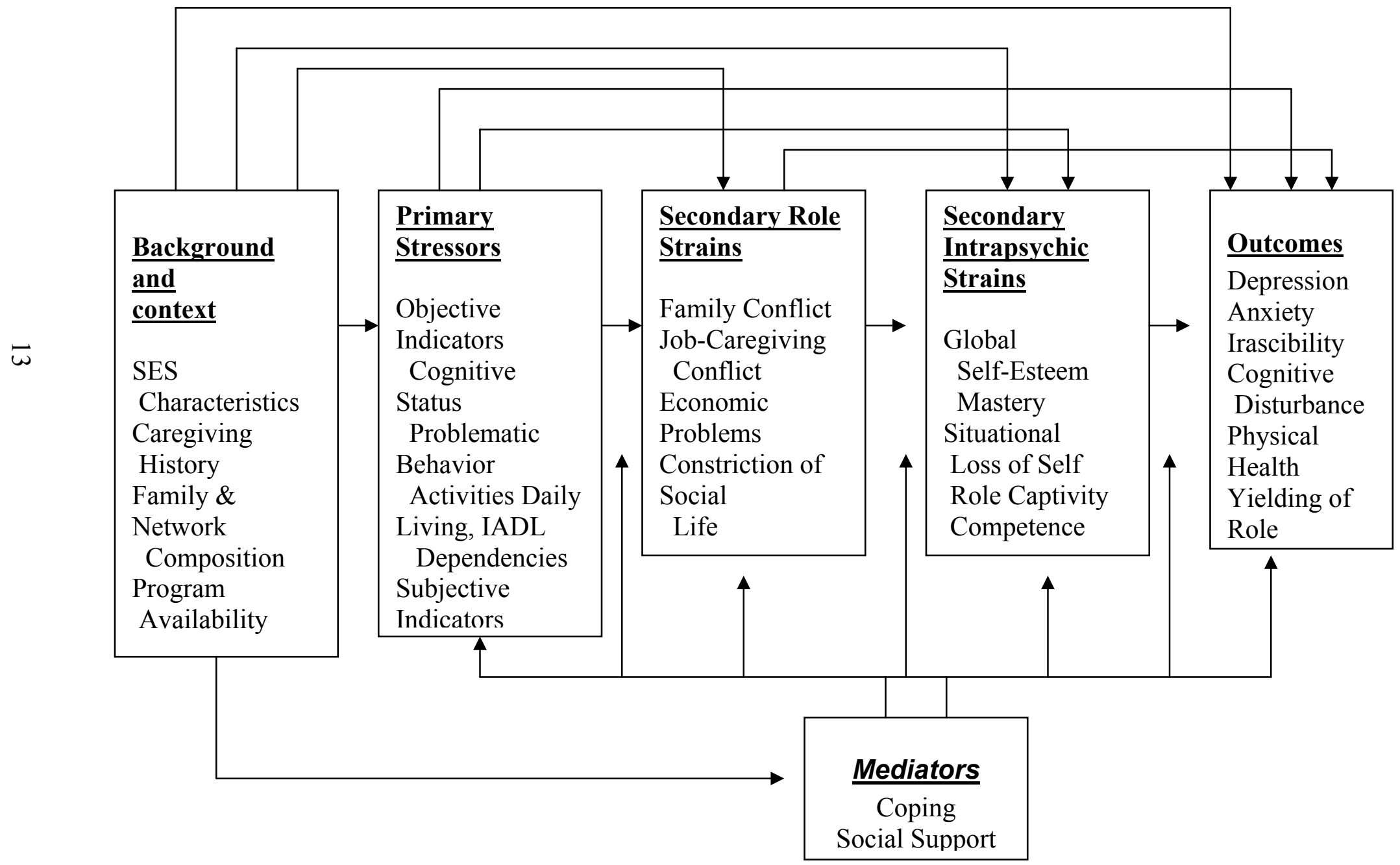


Figure 2. Conceptual Model Depicting Relationships among Primary and Secondary Stressors, Background and Contextual Factors, Patient Characteristics, Mediators, and Caregiver Stress Outcomes.

Note: Variables labeled with an asterisk were not tested in the prediction models. The dotted lines illustrated in the proposed model represent the relationships that were examined and the straight lines depict the relationships that were not examined within the current study.

Source: Pearlin, L., Mullan, J., Semple, S., \& Skaff, M. (1990). Caregiving and the stress process: An overview of concepts and their measures. The Gerontologist, 30(5), 583-594. Quoted in Harris, T. T. (2003). Burden and health in caregivers of persons with kidney disease. Unpublished doctoral dissertation, University of Tennessee, Memphis.

Copyright (C) 1990 by The Gerontological Society of America. Reprinted with permission of The Gerontological Society of America via the Copyright Clearance Center and Dr. T. Harris-Beard. 


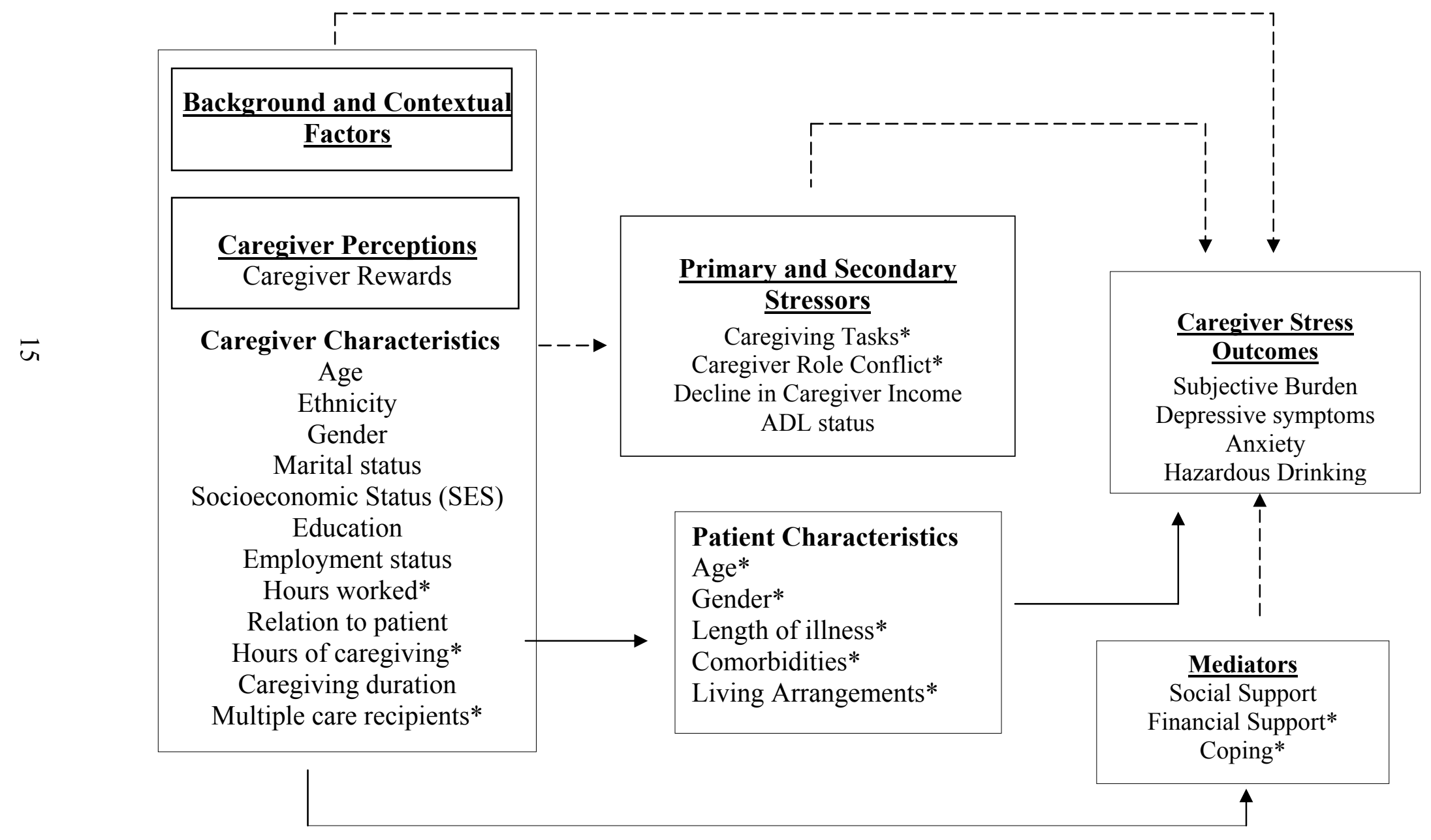




\section{Background and Context Components}

Attributes of the caregiver are major components of the background and context domain included within the SPM. Pearlin and colleagues (1990) contend that caregiver attributes such as age, educational level, gender, and ethnicity have a major effect on caregiving outcomes. These caregiver characteristics influence the type and intensity of stresses, the manner in which stress is expressed, and the ability to use personal and social resources. Little is known about the relationship between these attributes and caregiver outcomes in the context of CLD; however, study results about caregivers from other chronically ill populations support the utility of the model across patient diagnoses. In samples comprised of predominantly female caregivers assisting individuals diagnosed with cancer, ESRD, and stroke, significant burden occurred regardless of the diagnosis of the ill family member (Andrews, 2001; Belasco \& Sesso, 2002; Teel et al., 2001). Caregiver age has been studied as a correlate of burden in the context of chronic obstructive pulmonary disease and stroke; no correlation between age and burden was found in these studies (Cain \& Wicks 2000; Teel et al., 2001). The NFCA (1998) and Rees, O'Boyle, \& MacDonagh (2001) found that younger caregivers reported more burden; whereas, Shifren (2001) and Harris et al. (2000) found no significant difference in burden experienced by young versus older caregivers. Ethnicity, an attribute in the model, is a key variable in the proposed study. For example, burden and depressive symptoms in African American and Caucasian caregivers was compared in this study as inconsistent findings have been reported in other caregiver populations. Fredman et al. (1996) found no significant difference in burden while White et al. (2000) found that African American female caregivers reported fewer depressive symptoms than Caucasian 
female caregivers of an ill or disabled parent. These parents had physical illnesses as well as behavioral and memory impairments.

\section{$\underline{\text { Primary and Secondary Stressors }}$}

Caring for patients with CLD can produce a variety of stressors (psychological, physical and financial). Pearlin et al. (1990) identified two categories of stressors common to the experience of caregiving: primary and secondary (Figure 1). Primary stressors are directly related to the demands and needs of the care recipient and can lead to other stressors, which Pearlin and colleagues (1990) refer to as secondary stressors. An example of a primary stressor in family caregivers of individuals diagnosed with CLD is encephalopathy, which is a symptom frequently reported by patients with this illness. Encephalopathy causes cognitive changes, which interfere with the care recipient's ability to participate in self-care activities. An additional example of a primary stressor is the subjective hardship that is experienced by the caregiver. Subjective stressors are related to the perception of overload and relational deprivation that lead to feelings of burnout of the caregiver (Pearlin et al., 1990). Secondary stressors are related to role and intra-psychic strain, which encompass the effects that caregiving has on the ability to work and participate in outside activities and on one's psychological state. Pearlin et al. (1990) stated "having a close relative who needs care can certainly reawaken old family grievances and create new ones" (p.588). For an example, some individuals incurred CLD as a result of unhealthy lifestyles such as drug and alcohol abuse. Liver disease caused by destructive behaviors of the ill family member could cause conflicting feelings 
such as a sense of obligation versus anger. Another secondary role strain is economic strain caused by physical disabilities that may prohibit an ill family member from working. Some individuals diagnosed with CLD experience debilitating fatigue, which may lead to loss of employment and loss of income if the patient becomes disabled and loss of household income if patient hospitalization and general care needs necessitate caregiver absences from work. Intra-psychic strains are secondary stressors defined as dimensions of damaged self-concept influenced by continuous enduring hardship (Pearlin et al., 1990).

This study focused specifically on caregivers' mental health and subjective perceptions of burden and reward. Previous research examining mental health and burden in family caregivers have found significant correlations between perceptions of increased burden and debilitating symptoms, physical disability, and cognitive changes in the care recipients (Andrew, 2001; Belasco \& Sesso, 2002; Teel et al., 2001, Weitzner et al., 2000). Caring for individuals with CLD can be particularly stressful because these individuals often manifest severe symptoms such as encephalopathy and fatigue that may impair their ability to care for themselves and significantly impair interactions between the caregiver and care recipient (Habib et al., 2001).

\section{$\underline{\text { Mediators }}$}

In the SPM, Pearlin et al. (1990) describe mediating conditions or factors as the coping resources that may influence the caregiver's perceptions of the caregiving experience. Coping is described as "behaviors and practices of individuals as they act on their own behalf" (Pearlin et al., p.590). Social support is the second mediating factor 
identified by Pearlin et al. (1990) and is the assistance that is available to the caregiver. Coping and social supports mediate primary and secondary stressors and are therefore buffers and prevent or reduce the stress associated with the caregiver role (Pearlin et al., 1990). Hazardous drinking is an ineffective coping mechanism that may affect the caregiving experience; therefore hazardous drinking is the coping mechanism examined in this study.

\section{$\underline{\text { Outcomes }}$}

Outcomes are the last components of the SPM that "involve the well-being of people, their physical and mental health" (Pearlin et al., p.590). As shown in Figure 1, the specific outcomes that the model identifies are depression, anxiety, irascibility, physical health, cognitive disturbances, and yielding of role. Depression, anxiety, and problem drinking are investigated in this study as key outcome variables. Difficulties associated with caregiving such as isolation, loss of productivity, depression, and stress are identified as symptoms consistent with caregivers' burden (NFCA Report, 1998). Even though caregiver rewards are not a component of the SPM, they will be assessed in this study. Pearlin and colleagues (1990) stated "there are caregivers of course who manage to find some inner enrichment and growth even as they tend with mounting burden" (p.584). Picot et al. (1995) studied rewards, costs, and coping of African American caregivers and found costs and rewards were not significantly correlated and that a high level of rewards was reported. Thus, assessing caregiver rewards as an outcome is consistent with Pearlin's et al. (1990) conceptions of the caregiving process. 
The conceptual framework that guided this study incorporated several domains described within Pearlin et al. (1990) stress framework. Figure 1 depicts the relationships between primary and secondary stressors (i.e. caregiving income decrease and activities of daily living), background and contextual factors, mediators, and caregiver stress outcomes. The proposed model includes specific factors within each domain that could influence caregiver outcomes (Figure 2). Factors identified with an asterisk were not tested in the model. The dotted lines in the proposed model represent the relationships that were examined and the straight lines identify the relationships that were not tested.

\section{Definitions of Major Concepts}

The SPM (Figure 1) depicts the relationships among patient characteristics, contextual factors, primary and secondary stressors, mediating conditions, and outcomes. Several domains described within this model are incorporated within the conceptual framework used in this study (Figure 2). The key theoretical definitions used in this study are described.

\section{Depressive Symptoms}

Depressive symptoms refer to experiences and feelings indicating that individuals may have a depressive disorder which is an illness that involves the body, mood, and thoughts. It affects the way a person eats and sleeps, the way one feels about oneself, and the way one thinks about things (http://www.nimh.nih.gov/publicat/depressionmenu .cfm). These symptoms include feelings of sadness, anxiety, hopelessness, loss of energy, feelings of guilt, worthlessness, persistent physical symptoms, difficulty concentrating, 
irritability, insomnia, and thoughts of death (http://www.nimh.nih.gov/publicat/ depressionmenu.cfm). The CES-D, designed to measure current levels of depressive symptoms with emphasis on the affective component, was used to measure depressive symptoms in this study. A score of 16 on the CES-D is indicative of significant depressive symptoms (Radloff, 1977).

\section{Anxiety}

The definition of anxiety used in this study is consistent with the National Institute of Health's definition (http://www.nimh.nih.gov/publicat/anxiety.cfm\#anx7, 2004). The symptoms of anxiety include chronic and exaggerated worry and tension. There are thoughts of pending disaster. There is excessive worry and tension about health, money, family, or work. Excessive worry is also accompanied by physical symptoms, especially fatigue, headaches, muscle tension, muscle aches, difficulty swallowing, trembling, twitching, irritability, sweating, and hot flashes. People with General Anxiety Disorder (GAD) may feel lightheaded or out of breath. Anxiety was measured with the Hamilton Anxiety Rating Scale (HAM-A), which consists of 14 items, each defined by a series of symptoms that quantifies anxiety symptoms. A score of 18 on the HAM-A indicative of mild anxiety, a score of 25 represents moderate anxiety and 30 is severe anxiety (Hamilton, 1959).

\section{Hazardous Drinking}

The World Health Organization (1993) recognizes hazardous drinking as a distinct disorder. The disorder is defined as a quantity or pattern of alcohol consumption 
that places an individual at risk for adverse health events. The pattern of consumption that defines hazardous drinking is the consumption of 21 drinks or more per week for men and 14 drinks per week for women. The Alcohol Use Disorders Identification Test (AUDIT), a questionnaire consisting of 10 questions including items assessing alcohol consumption, problems, and dependency (Saunders et al., 1993), was used to measure hazardous drinking. A score of 8 or more suggested a potential drinking problem (Reinhart \& Allen, 2002).

$\underline{\text { Burden }}$

Burden refers to the negative psychological, economic, and physical impact of caring for an impaired individual ((Zarit, Todd, \& Zarit, 1986). Pearlin et al. (1990) define objective burden as the stress associated with the tasks and activities related to providing care. The impact of caregiving is referred to in the literature as caregiver burden, stress, and strain. For this study, subjective burden was measured using the Burden Interview (Zarit et al., 1986).

\section{$\underline{\text { Subjective Burden }}$}

Subjective burden is the caregiver's perception of burden and the caregiver's emotional reaction to the caregiving experience (Montgomery et al., 1985; Biegel, Sales, $\&$ Schultz, 1991). Subjective burden was measured using the 25 -item subjective burden subscale of the Burden Interview (Zarit et al., 1986). Scores from 0 to 20 is indicative of little or no burden, 21 to 40 mild to moderate burden and 61 to 88 severe burden (Zarit et al., 1986) 


\section{$\underline{\text { Caregiver Rewards }}$}

Caregiver rewards include positive feelings or gains felt by the caregiver (Given et. al, 1988). Caregiver rewards were measured with the Picot Caregiver Rewards Scale (PCRS). The PCRS is a 24-item scale that includes "caregiver perceived pleasures, satisfactions, good feelings, and consequences" (Picot, 1995; p.149).

\section{$\underline{\text { Characteristics }}$}

Attributes such as age, gender, and ethnicity along with educational, occupational, and economic attainments are integral components of the SPM and was measured in this study.

\section{$\underline{\text { Caregiver Characteristics }}$}

Characteristics are self-report information about the caregiver that include age, educational level, income, gender, relation to patient, caregiving duration, and hours providing care, and if caregivers are providing care for someone other than the care recipient with CLD.

- Ethnicity refers to the identification of a group based on perceived cultural similarities and group affiliation.

- Age was determined by subtracting the present year from the participant's date of birth.

- Educational level was defined as number of years of formal education. 
- Income was defined as total incoming finances that support the entire family on an annual basis.

- Gender was self-reported as male or female.

- Relation is identified as the caregivers' kinship to the care recipients.

- Caregiving duration was the length of time that the caregiver has provided care since the beginning of the care recipients' illness in months.

- Hours spent providing care refers to the time amount of time the caregiver provided care within a 24 hours period.

- Caregiver providing care for someone else referred to the number of individuals such as infants, school age children, elderly or frail relatives the caregivers assisted excluding the care recipient with CLD.

\section{$\underline{\text { Patient Characteristics }}$}

Patient characteristics in this study included age, gender, length of caregiving, severity of symptoms, and living arrangement.

- Age was determined by subtracting the year, at time of data collection, from the participant's date of birth.

- Gender for the care recipient was categorized as male or female and reported by their caregiver.

- Disease severity was measured using the Child Pugh Score (CPS). The CPS is a marker of liver disease severity and mortality (Forman \& Lucey, 2001). The CPS measures five criteria: total serum bilirubin, serum albumin, and ionized normalized ratio (INR) levels and the degree of ascites and encephalopathy. The 
scale predicts severity of liver disease, life expectancy, and liver transplant candidacy status (Riley \& Bhatti, 2001).

- Living arrangement referred to the person with whom the care recipient spent most of his or her sleeping hours.

\section{$\underline{\text { Chronic Liver Disease }}$}

Chronic liver disease is caused by varying degrees of inflammation to the liver as a result of illnesses such as hepatitis $\mathrm{C}$. Hepatitis $\mathrm{C}$ is the most common liver disease in the United States affecting approximately 170 million people (Koff, 1998; Patel, Muir, \& Hutchinson, 2006). Caregivers of patients who had a diagnosis of CLD were included in this study. A diagnosis of CLD was operationally defined by the care recipient's CPS, which includes ratings of their most recent bilirubin, albumin, and INR levels, and the presence of encephalopathy and ascites. These data were obtained from consenting patients' medical records.

\section{Concept Relationships}

Specific caregiver and patient characteristics interact to influence caregiver mental health and the magnitude of caregiver burden. As shown in Figure 2, variables within the domains of the conceptual framework are associated with caregiver burden and mental health status (Pearlin et al., 1990). Many studies indicate that most caregivers are female. However, men and women experience caregiving differently specific to the task that is involved. Women are reported to assist with more difficult tasks than men (Bedard, Koivuranta, \& Stuckey, 2004; Belasco \& Sesso, 2002; McCullagh, Brigstocke, 
Donaldson, \& Kalra, 2005; Pinquart \& Sorensen, 2006). Several researchers suggest that female caregivers experience significant subjective burden (Andrews, 2001; Belasco \& Sesso, 2002; Teel et al., 2001) that is more severe than the levels reported by male caregivers (Belasco \& Sesso, 2002; Chiroboga, 1998; Gallicchio et al., 2002; Nagatomo et al.; 1999). Some researchers found younger caregivers report more burden than older caregivers (Baronet, 1999; Cain \& Wicks, 2000; Dyck, Short, \& Vitaliano, 1999). Some research studies report that caregiver burden is perceived differently by various ethnic groups. African American caregivers are reported to experience lower levels of burden when compared to Caucasians (Janevic \& Connell, 2001; Knight, Silverstein, McCallum, \& Fox, 2000; Navaie-Waliser, Spriggs, \& Feldman, 2002; Roth, Haley, Owen, Clay, \& Goode, 2001; White et al., 2000). Other research studies report no difference in the levels of burden reported by African American and Caucasian caregivers (Teel, Duncan, \& Lai, 2001; Wood \& Parham, 1990; Young \& Kahana, 1995). No research has been conducted with caregivers of CLD; therefore, demographic characteristics of the caregiver and care recipient (diagnosed with CLD), caregivers' depressive symptoms, anxiety symptoms, hazardous drinking, subjective burden, and rewards are variables examined in this study. Caregiver characteristics found to influence subjective burden and mental health status are age, race, and relation to care recipient, gender, marital status, socioeconomic status (SES), education, hours spent in caregiving, duration of caregiving, and caring for someone other than the patient with CLD (Chiroboga et al., 1998; Kozachik, 2001: NFCA Report, 1998; Nijboer et a1., 1999).

Variables included in the domain of patients' characteristics include age, gender, length of illness, disease severity as measured by the CPS, and living arrangement. 
Research studies suggest that subjective burden is significantly associated with patient characteristics, such as length of illness, severity of symptoms, and patient dependency (Gallicchio, et al., 2002; Neundorfer et al., 2001).

Perceptions of caregiver burden are significantly correlated with caregivers' perceptions of the care recipients' symptoms and health. Researchers have found that caregivers who rated care recipients' health as poor experienced more burden than those who reported better care recipient health (Andrews, 2001; Karlawish et al., 2001). Thus, in the current study a similar association was expected

\section{Summary}

Previous studies indicate that the caregiving experience causes stress and burden for many caregivers especially when care recipients are diagnosed with a chronic illness that manifests severe symptoms. Chronic liver disease is an illness with debilitating symptoms that may require the assistance of a caregiver. Unalleviated stress in the caregiver could disrupt the caregiving process and contribute to caregiver mental health problems such as depressive and anxiety symptoms and the use of ineffective coping strategies such as hazardous drinking. Therefore, it is imperative to identify the factors that influence depressive symptoms, anxiety symptom levels, subjective burden, and rewards for family caregivers of CLD patients. The SPM is used in this study to provide a foundation that predicts the relationship between caregiver stress, characteristics, and outcomes. There are many studies addressing the effects of providing informal care to family members diagnosed with cognitive impairment. There are no published studies investigating the mental health status, prevalence of hazardous drinking, subjective 
burden, and rewards reported by caregivers of individuals diagnosed with CLD. This study addresses these gaps in the published literature, particularly the association between the caregiving experience and the mental health status of caregivers of individuals diagnosed with CLD.

\section{Significance of the Study}

Limited information exists in the literature about family caregiver alcohol use in any patient population. This study addresses that gap in research by investigating hazardous drinking in this sample. Information that is related to the prevalence of excessive drinking in family caregivers of individuals diagnosed with CLD is imperative because hazardous drinking places the family caregiver at risk for health problems and can disrupt the caregiving process potentially influencing patient outcomes. In several published studies the prevalence of alcoholism and other disorders such as depression and anxiety were higher among those family members who grew up or resided with a family member who was a problem drinker (Anda et al., 2002; Hurcom et al., 2000; Preuss, 2002). Therefore, some family caregivers of individuals diagnosed with CLD may be at risk for mental health problems (depressive and anxiety symptoms) and problem drinking. These areas are not addressed in the published literature describing caregivers assisting this patient population. Information obtained from this study will provide a foundation for future interventions to address the needs of this understudied population. Additionally, health care providers can assist family caregivers to determine the sources of their burden and the characteristics that place them at risk for increased burden and poorer mental health. Ultimately, the goal of this study is to assist health care providers 
to identify and then design interventions that assist family caregivers to better care for themselves thus preventing health problems and potentially disruptions in the caregiving process.

\section{Assumptions}

The following assumptions for this study were rooted in the conceptual framework and study design.

1. Providing care to a family member with CLD is a stressor and may create burden for some caregivers, especially since caregiver burden is associated with symptom distress. Some patients diagnosed with CLD often experience debilitating symptoms such as fatigue, encephalopathy, and cirrhosis (Andrew, 2001; Biegel, 1991; Bodenheimer et al., 2002; Roberts, 2000).

2. In the proposed study, burden is viewed as a multidimensional construct and because burden and mental health problems are outcomes of the caregiving process, it is important to understand the effects of caregiving on the caregiver. Caregivers assisting various chronically ill persons often experience both subjective burden and depressive symptoms (Kaneda \& Fujii, 2000; Kozachik, 2001, NFCA Report, 1998; Nijboer et a1., 1999; Pearlin et al., 1990).

3. Concepts depicted by Pearlin's SPM model are significant for family caregivers of persons with CLD. Patient and caregiver characteristics influence caregiver subjective burden and mental health outcomes across a broad group of patient populations. There are reasons to believe that this population of caregivers coping 
with CLD will have similar experiences (Andrew, 2001; Belasco \& Sesso, 2002;

Teel et al., 2001).

4. Some caregivers experience rewards associated with the caregiving role (Picot, 1995). Researchers suggest that caregivers who view the caregiving experience as rewarding often attribute spending time with the care recipient and having positive effects on the care recipient's health as the reason for the sense of gratification (Cohen, Colantonio, \& Venich, 2002; Sterrit \& Pokorny, 1998).

\section{Limitations}

The following are limitations of this study.

1. The CES-D is used as a self-assessment of mental health status, specifically a measure of depressive symptoms. The instrument, commonly used in caregiving studies, is a screening rather than a diagnostic tool to identify individuals at risk for depression. The CES-D descriptions for depressive symptoms are not consistent with the categories for depression in the DSM-III or DSM IV. However many investigators have assessed caregiver depressive symptoms using this scale. Use of the CES-D will assist with comparing the depressive symptom levels reported in the current study sample with those reported by caregivers assisting other patient populations.

2. Convenience sampling was an appropriate sampling method, because knowledge in this area of inquiry is limited. However, results must be regarded with caution because this study is descriptive and correlational in nature and a non-probability 
sampling (convenience sampling) design was used for this study limiting the external validity of study findings.

3. Most of the individuals with CLD were from metropolitan and southern rural areas; therefore, the results may not reflect the experiences reported by individuals who live in other geographic regions.

4. Persons in this study represent a specific group. Caregivers who are severely depressed and severely burdened are probably not represented in the study sample. Although the CES-D is a screening tool, individuals suffering from overwhelming lack of energy, hopelessness, guilt, and worthlessness are not likely to participate in the study. Additionally, the demands of caregiving may have prevented individuals who are severely burdened from participating in the current research study. 


\section{CHAPTER 2. REVIEW OF LITERATURE}

\section{Introduction}

Over the last ten years, published literature related to caregiving has expanded beyond informal caregivers of persons with cognitive impairments to patients with varied chronic diseases; however, the literature related to the experiences of caregivers of persons with CLD is limited despite the prevalence of the disorder. Researchers report that providing care for relatives with chronic illnesses such as Alzheimer's disease, cancer, and renal disease can cause psychological and physical strain (Belasco \& Sesso, 2002; Croog, Burleson, Sudilovsky, \& Baume, 2006; Given et al., 2004). Even though caregivers of patients with chronic diseases may share similar experiences, the current study focuses on caregivers of persons diagnosed with CLD because there are unique aspects to liver disease that effect caregivers in different ways. Individuals diagnosed with CLD may experience severe fatigue, pruritis, ascites, anorexia, nutritional deficits, and cognitive changes related to encephalopathy (Habib et al., 2001). Severe symptoms are likely to effect caregivers and are essential to the amount of strain experienced (Biegel, Sales, \& Schultz, 1991). Despite the severity of symptoms experienced by patients with CLD and their potential effects on caregivers, there are limited published studies examining the experiences of these caregivers. Information obtained from the current study with regard to mental health status, subjective burden, and caregiver rewards reported by family caregivers of patients with CLD will assist healthcare providers to develop appropriate interventions that are designed to maintain mental health in this caregiver population. Therefore, patient and caregiver background characteristics 
and caregiver stress outcomes (caregiver mental health status, reward, and subjective burden) will be described in caregivers of persons with chronic physical illnesses. A review of recently published studies relevant to the concepts within the SPM model is also included in this chapter. An overview of results of the literature related to general caregiving, caregiver characteristics, caregiver mental health and caregiver burden, caregiver rewards, caregiver characteristics and caregiver burden, caregiver age and caregiver burden, caregiver race and caregiver burden, caregiver gender and caregiver burden, caregiver relationship to patient and subjective caregiver burden, caregiver socioeconomic status and caregiver burden, caregiver support and caregiver burden, and caregiver characteristics and caregiver depression, anxiety, and substance use. A summary of the research related to patient characteristics and caregiver burden, which include patient's age, length of illness and living arrangement, is provided. The chapter concludes with a synthesis of the literature and a discussion of how prior studies inform the current study.

\section{Caregiving}

Caregiving refers to unpaid activities provided by family members or others that is frequently time-consuming, difficult and that extends beyond the customary support provided in a common relationship (Hauser \& Kramer, 2004; Hinton, 2002). Other researchers define caregiving more specifically as providing assistance with personal activities such as activities of daily living (eating dressing and toileting) and/or instrumental activities of daily living (assistance with cooking house cleaning and laundry (Ekwall, Sivberg, \& Hallberg, 2004; McCann et al., 2000). Caregiving is a 
complicated, multidimensional process with serious implications for the caregiver (Beach, et al., 2005; Scherling, 2002). Researchers have found that caregivers' experiences are influenced by the severity of care recipients' symptoms, caregiver characteristics, the length of caregiving experience, and social support perceived by caregivers (Brower et al., 2004; Karliswish et al., 2001; Given et al. 2004; NavaieWaliser, Spriggs, \& Feldman, 2002). Consequently, many caregivers report that the experience is stressful causing deterioration of their physical and mental health (Savage \& Bailey, 2005; Navaie-Waliser et al. 2002). When the caregiving experience is perceived as demanding, stressful, and severe it is conceptualized as caregiver burden.

Although caregivers respond to the needs of physically ill relatives, they do so with the risk of jeopardizing their physical and mental health. Investigators report that caregivers of persons with chronic illnesses in general face significant risks of declining physical and mental health status (Belasco \& Sesso, 2002; Harris, Thomas, Wicks, Faulkner, \& Hathaway, 2000; Lim \& Zebrack, 2005; Navaie-Waliser et al., 2002). Caregivers of persons with CLD probably face similar risks, however it is unclear if these risks vary by caregiver characteristics as reportedly occurs in other caregiver populations.

\section{Caregiver Characteristics}

According to the SPM, which is the framework for the current study, the caregiving experience is influenced by caregivers' characteristics. The key caregiver characteristics often associated with the caregiving experience are gender, age, race, socioeconomic status, and relationship (kinship) to the patient (Pearlin, 1990). Many researchers have investigated the influence of caregiver characteristics on the caregiving 
experience but report conflicting results. While many studies indicate that most caregivers are female, men and women experience caregiving differently specific to the task that is involved. Women are reported to assist with more tasks than men and tasks that are more difficult such as cleaning house, bathing the care recipient, and preparing meals whereas men are reported to provide transportation and assist the care recipient with mobility (Bedard, Kolvuranta, \& Stuckey, 2004; Belasco \& Sesso, 2002; Brazil, Bedard, Willison, \& Hode, 2003; McCullagh, Brigstocke, Donaldson, \& Lalit, 2005; Pinquart \& Sorensen, 2006). The caregiving experience is also affected by age and race. Some researchers found younger caregivers report more burden than older caregivers. Perhaps younger caregivers experience more burden because of competing responsibilities, such as caring for young children (Baronet, 1999; Cain \& Wicks, 2000; Dyck, Short, Vitaliano, 1999). Older caregivers are oftentimes the spouse of the care recipient and experience caregiving differently because their health status may be poor or declining (McCann et al., 2000; Navaie-Waliser et al., 1999). The caregiving experience is also influenced by culture, thus ethnic differences in the caregiving experience have been reported. Some researchers' report African Americans are more likely to report less burden and depression when caring for an ill relative and more reward and satisfaction (Connell \& Gibson, 1997; Haley, Roth, Coleton, Ford, \& West, 1996; Janevic \& Connell, 2001; Knight et al., 2000). These differences are reported to be influenced by African Americans coping with caregiving difficulties with prayer, faith, and religious practices (Dilworth-Anderson, 2001; Janevic \& McConnell, 2001 Picot, 1995; Picot et al., 1997). When researchers studied religious practices and spiritual influences on burden, African Americans were found to report higher levels of spiritual well-being and comfort from 
prayer and religion (Picot, Debanne, Namazi, \& Wykle, 1997; Spurlock, 2005). Other researchers report there is no significant difference in the African American caregivers' experiences of burden and depression when compared to Caucasian caregivers (Teel et al., 2001; Wood \& Parham, 1990; Young \& Kahana, 1995). Moreover, African American caregivers are more likely to suffer a decrease in pay from unemployment because of responsibilities of caregiving (Bullock, Crawford, \& Tennstedt, 2003; Covinsky et al., 2001). The experience for minority caregivers may be affected by family values, resources, and socioeconomic status (Hinton \& Levkoff, 1999; Wallsten, 2000a)). Williams et al., (2003) reported that caregivers who were identified as having lowincome reported increased feelings of powerlessness and loneliness when compared to caregivers with higher incomes. Powerlessness and loneliness could increase the risk of mental health problems.

\section{$\underline{\text { Caregiver Mental Health }}$}

The caregiving experience affects caregivers' mental health status. Caregivers report a significant increase in negative mental health symptoms as a result of providing care for relatives (Polen \& Green, 2001). Examples of negative mental health symptoms include depression, anxiety, and substance abuse (Navaie-Waliser et al., 2002; Polen \& Green, 2001; Tsai \& Jirovec, 2005). Caregivers providing 36 hours of care or more are more likely to report symptoms of anxiety and depression (Cannuscio et al., 2003). Anxiety often co-occurs with depression (Fawcett, 1997; Kaneda \& Fujii, 2000; Nutt, 1997; Zimmerman, 2003). Although studies have shown an association between depression and anxiety in the general population, few studies examine the presence of 
anxiety and co-morbid depression or depressive symptoms in caregivers. Researchers show a correlation between increased depression and heavier alcohol consumption in women (Dixit, \& Crum, 2000; Schutte, et al., 1997). Since most caregivers are women, determining the extent of drinking in this population is important but, few studies have examined substance abuse in the context of caregiving. Individuals under significant negative stress may resort to substance abuse as a coping mechanism (King, Bernardy, \& Hauner, 2003). Therefore, it is plausible that caregivers experiencing significant burden, anxiety, or depressive symptoms may resort to substance abuse, particularly alcohol use, to relieve stress or significant burden (Polen \& Green, 2001).

\section{$\underline{\text { Caregiver Burden }}$}

Caregiver burden is defined as the negative psychological, physical, and economic consequence of providing care for a family member (Harris-Beard, 2003; Zarit, Todd, \& Zarit; 1986). Caregiver burden is a dichotomous concept and is categorized as subjective and objective burden. Subjective burden is the caregiver's negative perceptions and feelings related to the caregiving experience (Cain \& Wicks, 2000; Chou, 2000; Wicks, Milstead, Hathaway \& Cetingok, 1998). Researchers and caregivers often define caregiver burden synonymously with caregiver stress or strain (Pearlin et al., 1990). Objective burden refers to the activities associated with negative caregiving experiences. Subjective burden is the focus of the current study. Caregiver burden is a multifaceted phenomenon that has negative psychological effects for caregivers and care recipients (Chou, 2000; Brouwer et al., 2004; Ekwll, Sivberg, \& Hallberg, 2004). The level of burden has been documented in many caregiving populations. Many of these 
studies focused on burden and stress in caregivers of individuals with Alzheimer's disease and other types of dementia. Some investigators found significant associations between behavior problems (symptoms) of the care recipient and increased subjective burden (Bedard et al., 2004; Robinson, Adkisson, \& Weinrich, 2001; Sisk, 2000; Zarit et al., 1986). In studies investigating caregiver burden in caregivers of patients with Alzheimer's disease, amyotrophic lateral sclerosis, strokes, cancer, and AIDS, burden was significantly and positively correlated with disease severity and patients' disability (Andrews, 2001; Chio, Gauthier, Calvo, Ghiglione, \& Mutani, 2005; Donelan et al., 2002; Flaskerud, Carter, \& Lee; McCullagh, Brigstocke, Donaldson, \& Kalra, 2005; Teel, Duncan, \& Lai, 2001). Studies of caregivers of patients with arthritis indicated that subjective caregiver burden is common among partners of individuals with rheumatoid arthritis and is significantly correlated with length of time caring for their spouses, lack of family support, and disrupted schedule (Brouwer et al.2004; Jacobi et al, 2003). Mild to moderate subjective caregiver burden was found in caregivers of patients following a renal transplantation and was inversely correlated with social support (Wicks, Milstead, Hathaway, \& Cetingok, 1998). Caregivers of patients on renal dialysis who reported higher subjective burden were receiving less social support (Belasco \& Sesso, 2002; Ude, Valdes, Estebanez, \& Rebollo, 2004). No published studies were retrieved that investigated subjective or objective caregiver burden in caregivers of persons with CLD despite the fact that the disease is chronic, life threatening, and disabling (Habib et al., 2001). Patients with CLD may need the assistance of a family caregiver. While there are factors that are specific to the CLD population that likely create challenges for these caregivers, these caregivers may also experience rewards associated with the role. 


\section{Caregiver Rewards}

Published research documents that caregivers often suffer negative consequences from caregiving; however, some caregivers report positive benefits from the experience. Positive feelings or obligations felt by the caregiver are known as caregiver rewards (Cohen, Colantonio, \& Venich, 2002; Picot, 1995). Race, gender, religiosity, duration of caregiving, relationship to the patient and resources have been found to influence the perception of caregiver rewards. African American caregivers report more rewards when compared to Caucasians. Investigators state that African American caregivers report greater use of religious practices as a means of coping, which accounts for these between group differences (Picot et al. 1997; Picot, 1995). Caregiver benefits are inversely related to the care recipients' functional ability (Motenko, 1989; Picot, 1995; Picot et al., 1997). Additionally, caregiver reward is a possible mediator for negative consequences, such as burden (Picot et al., 1997; Roff et al., 2004). The inability to identify a positive aspect of the caregiving experience could be a risk factor for depression and poor health outcomes (Cohen, Colantonio, \& Venich, 2002; Motenko, 1989). Caregiver reward is an important phenomenon that could effect the overall perception of the caregiving experience and thus influence caregiver health outcomes and the experience of subjective burden.

\section{$\underline{\text { Caregiver Characteristics and Caregiver Burden }}$}

This section provides a critical overview of the literature related to caregivers' characteristics, particularly with regard to how these characteristics are related to caregiver burden as an important study outcome. There are no studies investigating caregivers of patients with CLD, thus this section focuses on studies that explored the 
relationship, caregiver characteristics and caregiver burden in other (non-CLD) caregiver populations. Although each disease is unique with regard to its effects on the caregiver there may be some similarities between experiences of caregivers assisting persons with CLD and caregiver populations assisting persons with other chronic diseases. An examination of these non-CLD caregiver studies could therefore inform the current study.

\section{Caregiver Age and Caregiver Burden}

Some studies have shown that characteristics of the caregiver including age may have an affect on caregiver perceptions of burden; however, few studies have been conducted specifically comparing the experiences of young versus older caregivers. Early seminal study findings indicate that younger female caregivers report more negative outcomes compared to older caregivers (Carey, Oberst, McCubbin, \& Hughes, 1991; Zarit, Todd, \& Zarit, 1986). In a study investigating caregiver attributes in chronic obstructive pulmonary disease, when the criteria for older caregivers was changed to 55 instead of 60 years of age the caregivers that were younger (55 years old or less) reported higher burden scores (Cain \& Wicks, 2000; Vitaliano, Russo, Young, Becker, \& Maiuro, 1991; Zarit et al., 1986). In a sample of caregivers of patients diagnosed with AIDS, dementia, and advanced staged cancer, the caregivers caring for the individuals with AIDS reported more stress and were significantly younger when compared to caregivers for the individuals with dementia and cancer (Flaskerud, Carter, \& Lee, 2000). In contrast, researchers specifically investigating burden in younger and older caregivers found no difference in subjective burden by caregiver age (Croog et al., 2006; Harris, Thomas, Wicks, Faulkner, \& Hathaway, 2000; Shifren, 2001; Teel et al., 2001). 
However, Schwarz and Roberts (2000) found that in older caregivers, age was related to less strain. The research related to age and its relationship with burden is limited and the results are mixed thus indicating that this relationships warrants examination in the current study sample.

\section{Caregiver Ethnicity and Caregiver Burden}

The effect of ethnicity on the caregiving experience is equivocal. In recent studies, African American caregivers are reported to experience lower levels of burden and stress when compared to Caucasians (Janevic \& Connell, 2001; Knight et al., 2000; Navaie-Waliser et al., 2002; Roth et al., 2001; White et al., 2000). Seminal earlier studies validate these results (Fredman, Daly, \& Lazur, 1995; Hinrichsen \& Ramirez, 1992; Lawton, Rajagppal, Brody, \& Kleban, 1992; Macera et al., 1992). In contrast, other investigators report that there was no difference in the levels of burden reported by African American and Caucasian caregivers (Teel et al., 2001; Wood \& Parham, 1990; Young \& Kahana, 1995). These inconsistencies may be due to low participation of African Americans in some research studies. Studies that typically indicate ethnic differences in burden include significant numbers of African American patients, suggesting that these studies were sufficiently powered to detect between group differences (Dilworth-Anderson, Williams, \& Gibson, 2002). Study results indicate that African Americans more often rely on emotion-focused coping instead of action-focused coping, which increases the risk of increasing strain and stress (Knight et al., 2000). Contextual vulnerabilities such as socioeconomic, health, and social risks predispose 
African American caregivers to higher stress and burden (Chadiha, Adams, Biegel, Auslander, \& Gutierrez, 2004).

\section{$\underline{\text { Caregiver Gender and Caregiver Burden }}$}

Investigators generally concur that most informal caregivers are women.

Moreover, females typically report more burden and stress than males when caring for individuals with a variety of chronic illnesses such as dementia, rheumatoid arthritis, amyotrophic lateral sclerosis, ESRD, and cancer (Belasco \& Sesso, 2002; Bookwala \& Schultz, 2000; Brouwer et al., 2004; Chio, Gauthier, Calvo, Ghiglione, \& Mutani, 2005;

Chou, 2000; Donelan, Falik, \& DesRoches, 2001; Gallicchio, Siddiqi, Langenberg, \& Baumgarten, 2002; Given et al., 2004; Navaie-Waliser et al., 2002; Pinquart \& Sorensen, 2006; Sarna \& Chang, 2000; Tsai \& Jirovec, 2005; Yee \& Schultz, 2000). These differences are likely the result of between group differences in the types of care provided. Women are more likely to provide more intensive and complex care such as assistance with activities of daily living, dressing changes to wounds in addition to traditional responsibilities such as cooking and cleaning for dependent relatives. Researchers report that men are more likely to provide transportation to appointments and for shopping trips (Amirkhanyan \& Wolf, 2003; Kneipp, Castleman, \& Gailor, 2004; Navaie-Waliser, Spriggs \& Feldman, 2002). In contrast, Bookwala and Schulz (2000) reported that men participated in caregiving activities similar to women, particularly if the men were the spouses of the care recipient. Despite the similarities reported in this study with regard to caregiving activities, men still reported less depressive symptoms than women. However, in other studies that included caregivers of patients with strokes 
and persons with other chronic conditions the level of burden was not correlated with gender (Annerstedt, Elmstahl, Ingvad, \& Sven-Marten, 2000; McCullagh et al., 2005; Teel et al., 2001; Wallsten, 2000b).

Although it is unclear if gender differences in the level of burden exist, published research consistently suggests that most caregivers were females and that caregiving tasks vary by caregiver gender. The task in terms of its nature, time requirement, and meaning for caregiver may influence caregiver outcomes. Gender is potentially a significant predictor of caregiver outcomes and thus warrants examination in the study population.

\section{Caregiver Relationship to Patient and Subjective Caregiver Burden}

The association between caregiver subjective burden and caregiver relationship is unclear. Findings of several studies indicate that most caregivers are spouses (Belasco \& Sesso, 2002; Blake, Lincoln, \& Clarke, 2003; Croog, Sudilovsky, Burleson, \& Baume, 2001; Navaie-Waliser, Spriggs, \& Feldman, 2002; Pinquart \& Sorensen, 2006; Wallsten, 2000). Spouses often score higher on burden scales than other relatives (Beach et al.2005; Bedard, Kouivuranta, \& Stuckey, 2004; Belasco \& Sesso, 2002; McCullagh, Brigstoke, Donaldson, \& Kalra, 2005). Other investigators did not find significant differences in burden reported by daughters and spouse caregivers (Seltzer \& Li, 1996; Lawrence, Tennstedt \& Assmann, 1998; Zarit, Keever, Bach-Peterson, 1980) perhaps because both group were predominantly women. Conversely, in another study investigating family caregivers of patients diagnosed with cancer at the end of life, non-spouses and daughters reported more burden than spouses. Burden was measured with subscales of the 
Caregiver Reaction Assessment instrument (Given et al., 1992), which measured the impact of providing care on the caregiver's daily schedule. The non-spouses and daughters were younger than spouse caregivers, which may have confounded study results related to varied roles or relationships. Younger caregivers are more likely to be less established in their careers and may inherently suffer higher burden as a result of caregiving responsibilities that interfere with career goals. Moreover, the small sample size of non-spouses and daughters $(26 \%)$ in the study may account for the inconsistencies in results (Given et al., 2004). Finally, differences in the conceptual definition of caregiver burden and the instrument used to measure burden may explain these inconsistencies in outcomes across studies.

\section{$\underline{\text { Caregiver Socioeconomic Status and Caregiver Burden }}$}

Limited research exists examining the relationship between socioeconomic status and burden. However, several investigators found that caregivers with low incomes were more likely to report increased burden, stress, and loneliness than those with high incomes (Belasco \& Sesso, 2002; Kneipp, Castleman, \& Gailor, 2004; Nijboer, Triemstra, Tempelaar, Sanderman, \& Van den Bos, 1999; Williams et al., 2003). One study specifically compared demographics, hours spent caring for the care recipient, and subjective evaluation of the experience between low income $(n=25)$ and not low income $(n=19)$ informal caregivers. Low income was defined as $\$ 25,000$ or less per year in the study. Results from this study were tentative because the sample size was small, which limited the reliability and external of study findings (William et al., 2003). In a study exploring influences of mental health for caregivers of individual diagnosed with cancer 
$(\mathrm{N}=148)$, there were three ranges of income used to categorize socioeconomic status, $\$ 40,000$ and lower, $\$ 40,000-60,000$, and $\$ 60,000$ and more per year. Nijboer et al. (1999) found low income caregivers reported stronger negative effect on finances than those with higher income. Although income will be assessed in the current study, the variable decrease in income will be examined as appropriate as a predictor of caregiver outcomes because this decline in income may be more important than the actual income.

\section{$\underline{\text { Caregiver Support and Caregiver Burden }}$}

Research describes social support as services, education, information, or a lay or professional person providing assistance to family caregivers (Stolz, Uden, \& Willman, 2004). Caregivers who receive social support are less likely to report increased burden and support is viewed as a moderator for stress (Clyburn, Stones, Hadjistavropoulos, \& Tuokko, 2000; Haley, LaMonde, Han, Burton, \& Schonwetter, 2003; Mant, Carter, Wade, \& Winner, 2000; McCullagh et al., 2005; Nijboer et al., 1999; Polen \& Green, 2001; Savage \& Bailey, 2004). In contrast, caregivers of patients receiving homecare compared to caregivers of patients without homecare did not differ in caregiver strain and this form of social support did not affect the caregivers' perceived burden (Choo et al., 2003; M. Hecht et al., 2003; McNally, Ben-Shlomo, \& Newman, 1999; Tsai \& Jirovec, 2005). There is an inconsistent relationship between caregiver support and caregiver burden reported in published studies. The current study will focus on social support in a group of caregivers who have not been previously studied to determine if there is a relationship between social support for caregivers of relatives with CLD and mental health outcomes, subjective burden, and caregiver rewards. In the caregiver populations, 
a lack of social support could increase the likelihood of negative health outcomes such as an increased risk for depressive symptoms.

\section{Caregiver Characteristics and Caregiver Depression, Anxiety, and Substance Use}

Research studies validate that some caregivers experience depression and anxiety while caring for an ill relative (Clyburn, Stones, Hadjistavropoulos, \& Tuokko, 2000; Covinsky et al., 2003; Croog, Sudilovsky, Burleson, \& Baume, 2001; Grunfeld et al., 2004; Nijboer, Triemstra, Tempelaar, Sanderman, \& Van den Bos, 1999) and two studies did not find depression to be related to caregiver characteristics (Gallicchio et al., 2002; Nijboer et al., 1999). Depression and anxiety are significantly correlated with caregiver characteristics. Females experiencing more strain and burden were more likely to experience more depression (Given et al., 2004; Haley et al., 2003; Schulz et al., 2001; Schrag et al., 2004; Yee \& Schulz, 2000). Cannuscio et al. (2002) found that women who spent 36 hours or more providing care experienced more anxiety and depression. In contrast unlike most studies, males experienced more depression than females in one study (Covinsky et al., 2003). Spouses and wives who were caregivers experienced more depression than children (Beeson, Horton-Deutsch, Farran \& Neundorfer, 2000; Berg et al., 2005; Haley, LaMonde, Han, Burton, Schonwetter, 2003; Kozachik et al., 2001). The inconsistencies in the research related to these caregiver characteristics and caregiver depression may be related to other stressors that are gender specific. In most caregiving studies, female caregivers comprise the majority in the sample, and females spent more time caregiving and perform more demanding tasks such as attending to ADL and housekeeping (Amirkhanyan \& Wolf, 2003). Caregiving men participate in caregiving 
tasks that are less demanding such as transporting the care recipient to various medical appointments. Additionally men are more likely to seek outside formal assistance with caregiving responsibilities, which may significantly reduce the risk of mental health problems (Bookwala \& Schulz; 2000; Navaie-Waliser, Spriggs, \& Feldman, 2002; Pinquart \& Sorensen, 2006). There was no significant relationship between age, ethnicity, and depression in one study that included family caregivers of persons who were HIV positive. However, depression was related to low income (Flaskerud, Carter, \& Lee, 2000). In another study, older caregivers were less depressed than younger ones (Polen \& Green, 2001; Berg et al., 2005). However there was no relationship between gender, age, and depression and anxiety in other studies (Gallicchio et al, 2002; Garand et al., 2005; Nijboer et al., 1999; Teel; Duncan \& Lai, 2001). Caregivers of individuals with cancer and HIV reported more anxiety than caregivers of individuals with age-related dementia (Flaskerud, Carter, \& Lee, 2000). The caregivers in this study who found little private time for themselves were the most anxious. The study's limitations were the small sample size $(\mathrm{N}=27)$; however, significant group differences occurred despite this limitation (Flaskerud et al., 2000). African Americans reported less depression when compared to Caucasian caregivers in some studies (Dilworth-Anderson, Williams, \& Gibson, 2002; Covinsky et al., 2003; Haley et al.1995; Miller, 1995). In other studies, African Americans reported equal anxiety and depression when compared to Caucasians (Knight et al., 2000; White et al., 2000). Depression scores may vary across ethnic groups. African Americans are reported to express depressive symptoms differently than Caucasians, reporting more physical symptoms and insomnia rather than mood-related symptoms (Brown, Schulberg, \& Madonia, 1996a; Brown, Schulberg, Sacco, Perel, \& 
Houck, 1999b). Although the CES-D has one item related to sleep problems the other items are related to mood (Brown et al., 1996a; Brown et al., 1999b; Janevic \& Connell, 2001).These studies used small samples to investigate depression in the African American population which is problematic as findings may not reflect in a valid way the experiences of this population of caregivers. Larger study samples with African Americans from diverse backgrounds are needed to confirm or refute that depression varies by caregiver ethnicity.

Few studies have investigated substance use in caregivers. However, Polen and Green (2001) compared alcohol consumption in caregivers $(n=689)$ and non caregivers $(\mathrm{n}=4,851)$ recruited from a health maintenance organization and found no significant between group differences. In the two published studies found, none of the caregiver characteristics (age, gender, race, and SES) were associated with increased alcohol consumption (Cochrane, Goering, Rogers, 1997; Polen \& Green, 2001).

Research consistently indicates that females report more depression than males. However, research findings are unequivocal related to anxiety. Few studies examine caregiving patterns of substance use and abuse. The inconsistencies in research findings related to anxiety may be attributed to the use of a variety of self report instruments to measure this construct. The current study examines depressive symptoms and anxiety using reliable and valid instruments that are broadly used in caregiver populations.

\section{$\underline{\text { Caregiver Characteristic and Caregiving Rewards }}$}

Not all caregivers experience burden when caring for family members (Pearlin, 1991; Picot, Debanne, Namazi, \& Wykle, 1997). Findings of several studies show that 
reward, like caregiver burden, is related to caregiver characteristics. African Americans $(\mathrm{n}=391)$ reported receiving more rewards than Caucasians $(\mathrm{n}=255)$ in a sample of caregivers assisting persons over the age of 60 with varied types of illnesses and disabilities. Wives of men with dementia experienced more gratification and higher wellbeing than husbands (Motenko, 1989; Picot et al., 1997). Parents reported more satisfaction with the caring role than spouses, while spouses reported experiencing more satisfaction than adult offspring (Savage \& Bailey, 2004). The current study will examine the relationship of caregiver characteristics and rewards to ascertain if caregivers of persons with CLD are similar to other caregiver populations.

Research studies have shown inconsistent results with regard to the association between caregiver characteristics and burden. Age, race, gender, and relationship to the care recipient were proposed to affect the caregiver's perceptions of burden in the current study. Because some authors have found significantly greater burden in caregivers who are young, Caucasian, or have a low-income these relationships were explored in the current study.

\section{Patient Characteristics and Caregiver Burden}

Few studies report that patient's characteristics are significantly related to caregiver burden. Patients' characteristics explored in published studies include the severity of patients' illness and functional abilities. These were typically correlated to caregivers' burden and stress. Researchers found that greater disease severity and poor functional ability which is determined by the patient's need for assistance with ADL were associated with burden (Aguglia et al., 2004; Blake, Lincoln \& Clarke, 2003; 
Braithwaite, 2000; Brazil, Bedard, Willison, \& Hode, 2003; Caap-Ahlgren \& Dehlin, 2002; Covinsky et al., 2003; Harris, Thomas, Wicks, Faulkner, \& Hathaway, 2000; Given et al., 2004; Kozachik et al., 2001; Nijboer et al., 1999; Mcullagh, Brigstocke, Donaldson, \& Kalra, 2005; Schrag, Hovris, Morley, Quinn, \& Jahanshahi, 2005). Conversely, some researchers found no evidence of increased stress and burden among caregivers of severely disabled parents (Amirkhanyan \& Wolf, 2003). Moreover, researchers found increased burden in caregivers especially in wives caring for spouses with behavior problems (Bookwala \& Schultz, 2000; Covinsky et al., 2003; Gallicchio, Siddiqi, Langenberg, \& Baumgarten, 2002; Robinson, Adkinsson, \& Wienrich, 2001). Although the samples within these studies are similar and the researchers used the same instrument, relationship of the caregiver to the care recipients may explain these inconsistencies. Relationship of the caregiver to the care recipient is reported to influence the caregiver's perception of burden. Adult children are reported to respond differently than spouses and women respond differently than male caregivers (Zarit, Reever, BachPeterson, 1980, Burgener \& Twigg, 2002). Additionally, Amirkhanyan and Wolf, (2003), investigated the caregiving experience of adult children caring for parents with dementia at different time periods. Questions related to parents disability were posed a year before the publication which means that the adult children had been caring for the disabled parent for at least a year.

\section{Patient Age and Caregiver Burden}

Few recently published studies have evaluated the association between patient age and caregiver burden. A seminal study conducted by Coyne and Smith (1991) found that 
in caregivers assisting a spouse after a myocardial infarction that wives' distress was related to the patient age. When a myocardial infarction occurred in younger spouses, the caregivers were more distressed. However, most studies focusing on patient age and caregiver burden were conducted using samples of caregivers of mentally ill family members. Dyck, Short, and Vitaliano (1999) conducted a study examining patients with schizophrenia $(\mathrm{N}=82)$ and reported that younger patient age predicted higher burden. In contrast, Riedel, Fredman, and Langerberg (1998) found that patient age was not associated with burden in a sample of 200 caregivers of post rehabilitation patients 55 years and older. These findings are consistent with other studies that found no significant relationship between patient age and caregiver burden (Annerstedt et al., 2000; Crotty \& Kulys, 1986; Thompson \& Doll, 1982). Because published findings are unequivocal, the current study will explore the relationship between patient age and subjective burden. Riedel et al. (1998)and Crotty and Kulys (1986) used a researcher developed instrument to measure burden and failed to specify if the focus was subjective or objective burden. Annerstedt et al. (2000) used the Caregiver Burden Scale (Montgomery, Gonyea, \& Hooyman, 1985) to measure burden. Therefore, various measures were used to assess burden, which makes it difficult to compare findings across studies.

\section{Patients' Length of Illness and Caregiver Burden}

Few recent studies investigate the effects of patients' length of illness and caregiver burden. Providing assistance for over a year was significantly related to increased burden in caregivers of patients with multiple sclerosis and terminal illnesses (Brazil, Bedard, Willison, \& Hode, 2003; Rivera-Navarro, Morales-Gonzales, Benito- 
Leons \& Madrid Demyelinating Diseases Group, 2003). These studies included samples of $91-119$ white caregivers who were primarily female. The caregiving lengths of time did not have an impact on the level of caregiver burden in a study investigating caregiver's preparedness for the role of caregiving (Scherbring, 2002). A study investigating subjective and objective burden of the caregiving experience found that when the onset of caregiving was abrupt, duration of caregiving was a predictor of burden. Wives of patients diagnosed with dementia, heart disease, stroke, arthritis and diabetes reported fewer burden even though the care situation was longer (Pinquart \& Sorensen, 2006). For daughters participating in one study, duration of caregiving was not predictive of burden (Seltzer \& Wailing, 1996). Researchers used a variety of instruments to measure subjective burden. Most of the participants in these studies were Caucasian. One study was conducted in Madrid and most participants were natives of that country. The current study will use the Burden Interview scale (Zarit et al., 1986); its sound validity and reliability have been documented across caregiver populations. The current study will compare African American and Caucasian caregivers to capture the perspectives of two important groups.

\section{Patients' Living Arrangements and Caregiver Burden}

The research results are inconsistent related to the association of the patient's living arrangements and caregiver burden. Some researchers have reported that most female caregivers lived with their care recipient and living with care recipient is associated with negative effects (Donelan, Falik, \& DesRoches, 2001; Donelan et al., 2002; Savage \& Bailey, 2004). Objective burden was positively associated with residing 
with the ill relative (Baronett, 1999). Living with the patient contributes to burden since caregivers are more likely to be involved with ADL because of the close proximity. In contrast, Laidlaw, Coverdale, Falloon, and Kydd (2002) found no difference in levels of stress in caregivers of patients with schizophrenia who lived together versus apart. Caregivers who performed intense ADL and lived apart from care recipients were as likely to rate their tasks as difficult as caregivers who lived with the care recipient (Donelan et al., 2002). Caregivers may continue to provide certain kinds of care when the care recipient is living in an institution or their own home. The results of these studies are dissimilar. The participants in the current study will be asked if there was any assistance with ADL to determine if there was any relationship between caregivers' assistance with ADL and depressive symptoms, anxiety symptom levels, subjective burden and caregiver rewards.

\section{Summary of the Review of Literature}

While no studies were retrieved that related to caregivers of persons with CLD and burden, some studies did show that varying degrees of subjective and objective burden exist broadly across caregiver populations. Therefore, research is needed to investigate the level of burden and its relationship to key characteristics in this population of caregivers (Belasco \& Sesso, 2002; Brazil, Bedard, Willison, \& Hode, 2003; Brouwer et al., 2004; Chio et al., 2005; Harris, et al. 2000; Wicks et al. 1997). Currently no studies of caregivers of patients with CLD examined caregiver outcomes that are typically explored (depressive symptoms, anxiety levels, prevalence of hazardous drinking, subjective burden, and caregiver rewards). Most studies that do not include patients with 
CLD indicate that there are significant relationships between caregiver and patient demographic variables and burden. Although some relationships between caregiver characteristics (i.e., gender, age, and race) and caregiver outcomes are constant across studies, a number of caregiver characteristics (i.e. age, SES) and outcome associations in research findings are inconclusive. In some studies caregivers report experiencing depression while others report none. Females are reported to experience increased depression from the caregiving experience. Some research report caregivers experience anxiety others report the opposite. African Americans are reported to experience greater caregiver rewards while Caucasians are reported to experience less.

Consistently, results suggest that caregivers who are women, spouses, and Caucasians, or caregivers with greater task demands experience greater burden and depression. Caregivers experience increased levels of burden and depression if the patient resides in the same household or the patient's dependency level increases. Thus, the present study will address the relationships between caregiver characteristics (i.e., age, gender, ethnicity, education, employment status, relation to patient, and hours of caregiving) and caregiver's depressive symptoms, anxiety levels, prevalence of hazardous drinking, subjective burden and caregiver rewards to determine the relationships among these variables in this caregiver population. Few studies investigate the anxiety and hazardous drinking in the caregiving population. Furthermore, there is no published research focusing on caregivers of family members with CLD. The current study will investigate depressive symptoms, anxiety, hazardous drinking, subjective burden and caregiver rewards in caregivers of family members with CLD. 


\section{CHAPTER 3. METHODOLOGY}

In this chapter, the research methodology and design chosen to answer the research questions are discussed. The proposed sample, setting, instruments, procedures, statistical analyses, and the protection of human subjects are also included.

\section{Research Design}

This cross-sectional, descriptive correlation study was used to investigate family caregivers of persons diagnosed with CLD. The cross-sectional approach was used because there was no intent to measure caregiving experiences over time. A descriptive design was chosen for the current study to characterize one sample of caregivers of patients diagnosed with CLD. Few studies describe family caregivers of persons diagnosed with CLD and their experiences. This investigator used a descriptive approach in the current study because it was the best initial approach to determine if the family caregivers' experiences in the context of CLD are similar to and different than the experiences reported by populations caring for patients with other chronic disorders. A correlation design was included in this study to compare the differences in the experiences of African American and Caucasian caregivers and to determine if there was a relationship between selected caregiver characteristics and mental health outcomes.

\section{Sample and Setting}

This study included a convenience sample of 73 family caregivers recruited through contacts with patients receiving care in a university-based hepatology practice 
located in a large southern metropolitan city. The hepatologists in this practice care for patients from Tennessee, Mississippi, Missouri, and Arkansas. Patients cared for in this practice have the following diagnoses: biliary cirrhosis $(9 \%)$, chronic hepatitis necrosis (8\%), cirrhosis of the liver (9\%), and hepatitis C (7\%). Approximately 2330 patients were seen in the practice in 2003. Of the patients cared for in this practice, $61 \%$ were Caucasians, 21\% African Americans, 1\% Hispanics, and 17\% had an unknown ethnicity.

The Child Pugh Score (CPS) (see Appendix A) was used to determine the severity of liver disease in the care recipients. The score was originally used as a criterion for including caregivers in the current study. Inclusion criteria were amended to include all patients regardless of their CPS score. This approach was taken to increase sample size since little was known about caregivers of patients with CLD. The CPS is a scoring system that measures five criteria: total serum bilirubin, serum albumin, ionized normalized ratio (INR), ascites, and encephalopathy. Using the Child-Pugh classification system (1964), the results of the lab values were assigned to one of three levels. These levels were summed and scores then grouped into either class A, B, or C, where each increase in class by alphabet represents worsening liver function. Thus, Class A category is the least severe and $\mathrm{B}$ and $\mathrm{C}$ are more severe and indicative of the need for liver transplant evaluation and potential transplantation (Forman \& Lucey, 2001; Riley \& Bhatti, 2001). In Class A (5-6 points) the care recipients' life expectancy is 15-20 years. Care recipients categorized in Class B (7-9 points) have serious conditions warranting liver transplant evaluation. Recipients in class $\mathrm{C}$ category (10-15 points) have the most severe conditions warranting liver transplantation; these individuals have a life 
expectancy of 1-3 years. A summary of the aforementioned classification and scoring system is provided in Appendix A.

For the current study, the results of the care recipient's total serum bilirubin, serum albumin, and INR from their last office visit were retrieved from their medical record with their permission and recorded on a Microsoft Excel spreadsheet. The second component of the CPS involves an assessment of the degree of ascites and encephalopathy found by the physician during a routine appointment. A rating of 1 reflects none whereas ratings of 2 reflect mild, and 3 marked levels of both ascites and encephalopathy. The results of the assessments for encephalopathy and ascites were also obtained from the patients' medical records with their permission and recorded on the form used to calculate the CPS. The points for the five categories were summed for a total CPS score indicating severity of the disease. Caregivers included in this proposed study were caring for individuals categorized in class A, B, or C as measured by the CPS. These individuals were likely involved in anticipatory caregiving, which is defined as decisions and behaviors based on future needs; preventive caregiving, which involves monitoring; and supervisory caregiving, which involves checking up on the care recipient and arranging things or the providing assistance with ADL (Ekwall, Sivaberg, Hallberg, 2004). The Mayo for End Stage Liver Disease (MELD) is also one of the most frequently used measures to predict severity of liver disease (Forman \& Lucey, 2001). The numeric value of the MELD was also calculated.

The inclusion criteria for family caregivers to participate in the study was identified and listed below. Primary caregivers were the caregivers that the care recipient 
reported cared for them or would care for them if they could no longer care for themselves.

1. Age 18 years or older on last birthday.

2. Residing permanently in the United States and able to speak and understand English.

3. Willingness to participate in the study and provide verbal and written consent. Participants excluded from the proposed study were as follows:

1. Unable to speak or read English, because all instruments are provided for those persons for which English is their primary language.

2. Caregivers of care recipients less than 18 years of age.

3. Caregivers of care recipients diagnosed with CLD who are not being treated in the study site.

To ensure an adequate sample size and external validity of the study, 73 caregivers were recruited for this study. A power analysis was performed to determine the number of subjects needed to ensure a p-value equal to .05 using the statistical package Power Precision. The effect size was chosen according to Cohen's criteria (1998) for a moderate effect size. For multiple regression analyses, a moderate effect size of 0.15 was used. It is the smallest effect that would be important to detect the variance, in the sense that any smaller effect would not be of clinical or substantive significance. In the proposed regression model, no more than 4 independent variables were chosen for the model based on predictions from the SPM and previous research. Using an alpha set at .05 , the study had a power of 0.80 with four independent variables. 


\section{$\underline{\text { Instrumentation }}$}

In this study, six paper and pencil questionnaires that addressed the study's variables included in the research questions were given to participating caregivers to independently complete. The principle investigator provided assistance for participants as needed. Written permission to use copyrighted instruments in this study was obtained. Instruments that were self-administered were the: Family Caregiver Demographic Data Form, Center for Epidemiological Studies Depression Scale (CES-D), Hamilton Anxiety Scale (HAM-A), Alcohol Use Disorders Identification Test (AUDIT), Picot Caregiver Reward Scale (PCRS), and Burden Inventory (BI). A description of the content, reliability, and validity of each instrument is provided in the following section.

\section{Family Caregiver Demographic Data Form}

The Family Caregiver Demographic Data Form was developed to record caregiver and care recipient demographic characteristics (see Appendix B). This investigator developed form was a self-administered questionnaire that requested information such as the caregiver's age, gender, ethnicity, marital status, education, income, and relationship to the care recipient. The care recipient's CPS, MELD, age, gender, length of illness, comorbidities, and living arrangements were recorded on a portion of the form labeled care recipient's demographic data. Flesch Kincaid assessment indicated the reading level of the Family Caregiver Demographic Data Form is 6.3. Estimated time for completion of the demographic data form is 5-10 minutes. 


\section{Center for Epidemiological Studies Depression Scale (CES-D)}

The CES-D was used to measure the level of depressive symptoms in this study. The CES-D was developed by Radloff (1977) to measure current levels of depressive symptomatology in the general population (see Appendix C). The self-report instrument has 20 items that were selected from previous instruments used for screening depression including: Beck’s Depression Inventory (Beck et al., 1961), a scale developed by Raskins et al. (1969), Zung's (1965) depression scale, and the Minnesota Multi-phasic Personality Inventory (Eaton et al, 1994; MMPI, 1960). The items are designed to identify major symptoms of depression found in the literature (Radloff, 1977). The scale emphasizes the affective components such as "depressed mood, feelings of guilt and worthlessness, feelings of helplessness and hopelessness, psychomotor retardation, loss of appetite, and sleep disorder during the past week" (Radloff, 1977, p.386). To counterbalance the problems with response sets, there are both positive and negative items included in the scale. For example, some questions (items 4, 8, 12, and 16) are worded positively and reversed scored to discourage a response set (Radloff, 1977). The CES-D is a Likert-type scale that uses a rating of 0 to 4 with 0 being rarely or no symptoms and 4 meaning that the individual is experiencing symptoms all of the time over the past 7 days. The total score of the CES-D is the sum of all item responses and can range from 0 to 60 (Radloff, 1977). Scores of 0 - 15 are generally interpreted to indicate no depressive symptoms, 16 - 20 mild distress, 21 - 30 moderate distress, and 31 and over severe distress (Radloff, 1977).

Reliability and validity testing of the instrument has been conducted in a variety of samples. Internal consistency in a community sample ranged from 0.80 to 0.90 ; test- 
retest reliability with time intervals from one to 2 -weeks ranged from 0.40 to 0.70 (Devins \& Edwards, 1988; Eaton et al., 1994; Radloff, 1977). To demonstrate repeatability properties of the scale, three field-tests using structured interviews were performed. Estimation of reliability between the three community groups ranged from .85 to .90 coefficient alpha (Radloff, 1977). A factor analysis was used to test validity of the scale in the original sample. Radloff found that the patterns of factor loadings were consistent across groups. Generality of the scale across groups was demonstrated by comparison across age, sex, race, and educational subgroups. Radloff found a coefficient alpha of .80 and above in all subgroups; the subgroups did not differ from each other or the general population. The CES-D has been widely used in caregiving populations of care recipients diagnosed with cancer, stroke, and Alzheimer's disease (Knight et al., 2000; Nijboer et al., 1999; Teel et al., 2001). Kaplan and Boss (1999) used this instrument to measure levels of depressive symptoms in African American caregivers of individuals with dementia. An alpha reliability of .89 was found in spousal caregivers of patients institutionalized with a diagnosis of Alzheimer's dementia. A reliability of .85 was found in caregivers of patients with schizophrenia (Dyck, et al., 1999). A Cronbach alpha coefficient of .93 was estimated supporting the instrument's internal consistency reliability for the current study sample.

Limitations of the CES-D are that the instrument was developed to screen for depressive symptoms and is not as a diagnostic instrument (Radloff). Cutoff scores should be regarded with caution and only considered as an indicator of depressive symptoms rather than a diagnosis. Additionally, the scale items from the CES-D are not based on the current DSM IV criteria for depression. The reading level for the CES-D is 
$6^{\text {th }}$ grade. Estimated time for completing the CES-D instrument is 5-10 minutes (Sharp \& Lipsky, 2002).

\section{Hamilton Anxiety Rating Scale (HAM-A)}

The HAM-A, developed in 1959 by Max Hamilton, was designed to quantify symptoms of anxiety. The HAM-A, a self-report instrument, consists of 14 items (see Appendix D). Participating caregivers were asked to recall symptoms from the past week and rate each item on a 5-point scale, ranging from 0 (not present) to 4 (severe). In a study of 292 community adults, internal consistency was .92 (Guy, 1976). Additionally, a reliability and validity study of the HAM-A was conducted with 257 adolescents. The scale is a reliable and valid measurement for assessing anxiety and demonstrates excellent construct validity as it has a statistically significant relationship with other measures of anxiety (Clark \& Donovan, 1994). On the HAM-A, a score of 18 represents mild anxiety, a score of 25 represents moderate anxiety, and a score of 30 is severe anxiety. There are no studies in the literature using the HAM-A in the caregiving population. A Cronbach alpha coefficient of .93 was estimated supporting the instrument's internal consistency reliability for the study sample. A Flesch-Kincaid assessment of the HAM-A indicated a reading level of 12.0. The approximate length of time to complete the HAM-A is 5-minutes.

\section{Alcohol Use Disorders Identification Test (AUDIT)}

The AUDIT, a self report instrument used to measure frequency (see Appendix E), alcohol dependency, and problems caused by drinking, was developed by the World 
Health Organization (WHO) to identify individuals whose alcohol consumption was believed to be harmful to their health (Babor et al., 1992; Reinhert \& Allen, 2002; Saunders et al., 1993). The AUDIT distinguishes between at risk users and individuals identified as alcohol dependent. In the current study, the AUDIT was used to identify hazardous drinking in family caregivers and distinguish caregivers of individuals diagnosed with CLD who are at risk for alcohol related health problems. The hazardous drinking category is defined as a pattern of alcohol consumption that places the individual at risk for adverse health outcomes. Reid et al. (1999) identifies a pattern of consuming 21 drinks per week or 7 drinks or greater per occasion at least 3 times per week as hazardous drinking for men. For women, hazardous drinking is a pattern of consuming 14 drinks per week or 5 drinks or greater per occasion at least 3 times a week (Reid et al., 1999). Because of the differences in body water distribution, females have less water to absorb, dilute, and decompose alcohol; consequently, the effect of alcohol is greater in lower quantities.

The AUDIT, a 10-item screening instrument, was developed to assess alcohol intake (items 1-3), dependence (items 4-6), and adverse consequence (items 7-10) (Reinhart \& Allen, 2002). Items are rated from 0 to 4 with a score of 8 or more suggestive of a potential drinking problem (Reinhart \& Allen, 2002). The AUDIT is scored by totaling the values of the item responses. Scores range from 0 to 40 with a cutoff point of 8 indicating a potential drinking problem. A cutoff value of 8 yielded a sensitivity score of .90 for problematic drinking (Babor et al., 2001). Reliability and validity of the AUDIT have been tested in many clinical settings. Internal consistency in a sample of 166 primary care patients was $r=0.88$ (Daeppen et al., 2000). Dawe et al. 
(2000) also found the AUDIT to have good internal consistency (coefficient $=0.85$ ), a $90 \%$ specificity and sensitivity of $87 \%$ in detecting hazardous drinking in a sample of 71 patients diagnosed with schizophrenia. Reinart and Allen (2002) conducted a review of the literature with studies conducted from 1996 and later to examine the results of psychometric testing of the AUDIT. These authors found that the AUDIT had high internal consistency and test-retest reliability across studies. The review of literature suggests that this instrument has never been used in the caregiver population. To establish reliability for this instrument in the current study sample, a Cronbach alpha coefficient of .72 was calculated using the SPSS reliability analysis. A Flesch Kincaid assessment of the AUDIT indicated a reading level of the 7.2. The AUDIT takes approximately 5 minutes to complete.

\section{Burden Interview (BI)}

The BI, a 22-item self-report instrument was used to measure subjective burden (see Appendix F) (Zarit et al., 1985). Subjective burden is the burden that is perceived by caregivers, the affective experience of the caregiving experience (Montgomery, Gonyea, \& Hooyman, 1985). Two subscales comprise the BI, personal and role strain; however, only the total score was used in the current study. Total scale scores range from 0 to 20 (little to no burden), 21 to 40 (mild to moderate burden), 41 to 60 (moderate to severe burden), and 61 to 88 (severe burden) (Zarit, 1985). Several researchers have published reliability results of the BI scale. The internal consistency reliability score was $r=.79$ in an adult population of caregivers of older adults (Zarit, 1985). A Cronbach alpha coefficient of .88 was reported on the instrument in young and elderly Black caregivers 
of patients with end stage renal disease (ESRD) establishing internal consistency reliability in this sample (Harris \& Thomas, 2001). Vitaliano et al. (1991) reported an internal consistency of $\alpha=.91$ and test-reliability of $\alpha=.71$. To establish the internal consistency reliability, a Cronbach alpha coefficient of .88 was obtained for the BI in the current study sample using SPSS reliability analysis. The BI is reported to have a reading level of the ninth grade (Cain \& Wicks, 2000). The BI takes approximately 5 minutes to complete.

Picot Caregiver Rewards Scale (PCRS)

The PCRS assessed perceived rewards reported by caregivers' of patients with CLD (see Appendix G). The PCRS is a 25-item, self-administered scale that includes perceived pleasures and satisfactions, good feelings, and positive consequences associated with the caregiver role (Picot, 1995). Caregivers rate their perception of positive consequences on 5-point Likert-type scale of zero, not at all to 4, a great deal. Reliability for the PCRS is high as evidenced by an alpha coefficient of .86 in a sample of 85 black female caregivers of elderly dementia patients (Picot, 1995). Scores range from 0 to 64 for the PCRS with higher scores indicating the greater perceived reward. A Cronbach alpha coefficient of .89 was calculated using the SPSS reliability analysis for the current study sample. A Flesch Kincaid assessment of the PCRS indicated a reading level of the 5.4. The PCRS takes approximately 5 minutes to complete. 


\section{Procedure}

Permission to conduct the proposed study was obtained from the University's Institutional Review Board (IRB). Written and verbal approval was obtained from the medical director(s) and physicians of the practice site to recruit caregivers of the patients seen in the private clinic. A letter of approval from the IRB was provided (See Appendix J). The PI collected all study data.

The office staff provided the patients in the hepatologists' office with a brochure and directed those who were interested in participating or hearing more about the study to the principal investigator's (PI) desk. Patients who approached the researcher were asked to identify the person who cared for them or who would care for them if they could no longer care for themselves (their caregiver). If the patient identified a caregiver, the consenting process and the purpose of the study were explained to the patient. Patients were told that refusal to participate in the study would not affect the treatment that they received in the clinic. After all questions were answered an informed consent was signed, the patient's medical record was reviewed for the most recent CPS score. If patient's met the inclusion criteria and the caregivers were present, willing to participate, and sign informed consent, the caregiver was given the option of completing the questionnaires during the appointment and returning the survey to the PI before leaving the clinic. The PI provided an envelope with return postage for caregivers who chose to complete the questionnaires at home. These caregivers received a follow up telephone call from the PI within 24 hours after receipt of the questionnaires. The purpose of this call was to answer any questions they had regarding the completion of the questionnaire packet. 
If patients met the criteria for inclusion in the study and were not accompanied by the primary caregiver, the PI gave the patient a questionnaire packet to give to their caregiver. The packet included a copy of the informed consent, questionnaires, and the PI's business card including a cell phone number for any questions pertaining to the study. A follow-up telephone call within 24 hours was made to answer questions that participants had about the study. After the questionnaires were completed and returned, the PI reviewed the caregivers' responses. If missing data were present, the PI telephoned the participant within 48 hours to obtain their responses, reiterating that there were no wrong or right answers on the questionnaire items. Caregivers were given a $\$ 20.00$ gift certificate at the time that the completed questionnaires were returned in person. A $\$ 20.00$ gift certificate was mailed to caregiver within one week after return of completed questionnaires by mail.

All instruments were coded to protect the participants' confidentiality. The participants were assigned codes and told that only group data would be reported. To increase the response rate, a post card was mailed to caregivers who did not return the completed questionnaires within one week.

\section{$\underline{\text { Site Preparation }}$}

Flyers and a poster describing the study were prepared by the PI and placed in a strategic location in the physician's practice. This poster and accompanying pamphlets were designed to explain the purpose of the study and emphasize that refusal to participate in the study would not interfere with their treatment. The PI developed an IRB-approved pamphlet, which included a photo and business card of the PI. The 
pamphlets explaining the study were distributed by the staff at the appointment desk and placed in the physician's practice waiting area. The PI presented and left copies of published articles regarding caregiver burden and mental health in caregivers of patients with chronic illnesses to registered nurses at the data collection site 6 weeks prior to initiation of data collection. The PI provided a catered lunch for staff 2 weeks prior to data collection and discussed the articles and answered any questions from staff regarding the study.

\section{$\underline{\text { Data Analysis }}$}

The following statistical analyses were conducted to describe the study's sample and answer the study's research questions. Each aim and corresponding research question is presented prior to describing the data analysis.

\section{Analysis of Sample Characteristics}

Prior to answering the research questions, simple descriptive statistics were performed to describe the demographic characteristics of caregivers and care recipients. The univariate procedures provided details on the distribution of the variables such as mean, median, extreme values, frequency tables, and test of normality (i.e. whether data were normally distributed). Data that were not normally distributed (positively skewed) were $\log$ transformed to normalize the data. Pearson product moment and spearman correlations were computed on data to assess significant associations. 


\section{Specific Aim One}

Describe depressive symptom levels, anxiety symptom levels, prevalence of hazardous drinking, subjective burden, and caregiver rewards in family caregivers of persons with CLD.

Related research question 1: What are the depressive symptoms and anxiety symptom levels, prevalence of hazardous drinking, subjective burden, and caregiver rewards reported by family caregivers of patients with CLD?

The univariate procedure provided information about the distribution of the variables such as, mean, median, extreme values, frequency tables, and tests of normality to determine whether the data was normally distributed. The distribution of the data was assessed to determine whether parametric or nonparametric statistical analyses were used to analyze the data and answer the research questions.

\section{Specific Aim Two}

Compare the depressive symptom, anxiety symptom levels, hazardous drinking, subjective burden, and caregiver rewards reported by African American and Caucasian family caregivers of persons diagnosed with CLD.

Related research question 2: What are the difference in levels of depressive symptom, anxiety symptom levels, hazardous drinking, subjective burden and rewards reported by African American and Caucasian family caregivers of patients diagnosed with CLD?

Descriptive statistics including measures of central tendency were used to describe levels of depressive and anxiety symptoms, hazardous drinking, caregiver 
rewards and subjective burden of the sample. A Mann Whitney test was conducted as a nonparametric alternative to comparing depressive symptoms, anxiety symptom levels, and hazardous drinking levels in African Americans and Caucasian caregivers. An independent $t$-test was done to determine if there was a significant difference in subjective burden and caregiver reward between the two groups.

\section{Specific Aim Three}

Explicate the predictors of subjective burden and mental health status of African American and Caucasian family caregivers of persons with CLD.

Related research question 3: What are the predictors of subjective burden and mental health (depressive and anxiety symptom levels) of African American and Caucasian family caregivers of patients diagnosed with CLD?

Correlation studies involve the systematic investigation of relationships between variables (Burns \& Grove, 2001). Thus, statistical correlation methods were used to examine the relationships that existed between the independent variables (i.e., caregiver characteristics) and dependent variables (i.e., depression, anxiety, subjective burden, and rewards) in caregivers, prior to conducting stepwise regression analysis procedures.

Pearson's and spearman's correlation coefficients were used to determine the relationship between caregiver attributes and levels of subjective burden. A Shapiro-Wilk test of normality was conducted to test the distribution of the independent variables. Normality of the variables that are being correlated is an assumption of the multiple regression analysis. When the Shapiro-Wilk test value is close to 1 and the significance is above .05 the variable is not normally distributed. The variables depressive symptoms $(w=.94, p=$ 
$.001)$ and anxiety symptoms level $(w=.92, p=\leq .00)$ were right skewed. A log transformation was performed on these variables before entering them into the regression model. The predictors of caregiver burden were examined with the stepwise multiple regression analysis. Subjective burden $(w=.97, p=.25)$ and caregiver rewards $(w=.99$, $p=.93$ ) were normally distributed. Several regression equations were performed and analyzed before completing the final regression model depicting the predictors of each outcome (depressive symptoms, anxiety levels, subjective burden and caregiver rewards). The first step was to identify the independent variables in each domain (caregiver background and contextual factors, primary and secondary stressors, patient characteristics, and mediators as depicted in the study's model) that were independently associated with outcome variables (depressive symptoms, anxiety symptoms, subjective burden, caregiver rewards) using the Pearson's product-moment or Spearman correlation analyses (if the variable was not normally distributed), and stepwise multiple regression analyses to determine which variables were predictive of the dependent variable. Caregiver characteristic variables that were hypothesized as relevant within the Pearlin SPM or supported as significant in published studies or clinical practice and significant $(\mathrm{p}$ $\leq .05)$ were entered into a stepwise multiple regression models. Four independent variables were chosen for inclusion each model to ensure a power of 0.80 with a $p$ value of .05. The independent variables that were correlated with the specific outcome variable were entered into the regression equation. Next, stepwise multiple regression analysis was performed to ascertain which caregiver independent variables best predict the dependent variable. The computer program eliminated the independent variables that 
were not significantly predictive in the model. Regression diagnostic analyses were performed to rule out the presence of multicollinearity within each model.

\section{Consideration of Human Subjects}

Human subject considerations are related to consent for participation, potential risks to subjects, and protection of confidentiality. Informed consent was obtained from each caregiver after fully describing the study in the form of a verbal and written explanation of their right to give or withhold consent to participation. The informed consent documents were included with the instruments as a part of the study packet found in Appendix I and J. Care recipients were asked and consented for the investigator to review the medical records. Each caregiver and care recipient was assured that participation is strictly voluntary and that: (a) participation would in no way influence the health care they (the care recipient) receive from their provider; (b) they could choose to withdraw at any time during the study; and (c) response to each individual question is desired, but not insisted upon.

Potential risks to subjects were minimal for caregivers completing the questionnaires related to demographic information, depression, anxiety, hazardous drinking, reward, and burden. The only anticipated risks were minimal stress or uneasiness pertaining to self-disclosure or introspection from answering questions related to depression, anxiety, and drinking patterns. A letter of referral was provided for caregivers who scored 16 and over on the CES-D for depressive symptoms, 18 and over on the HAM-A for anxious symptoms, and 8 and over on the AUDIT for hazardous drinking. Another potential risk was related to loss of time that it takes to complete the 
instruments. The gift certificate was provided to offset the time associated with completing the questionnaire. The following measures were incorporated into the study to decrease this risk: (a) submission for study approval by the Institutional Review Board (IRB) of the University of Tennessee and (b) a statement encouraging free expression was made at the beginning of each interview that "There is no right or wrong answer to any of the questions because everybody is different. Your opinion is what is important".

Both direct and indirect benefits could be obtained from participation in the study. An indirect benefit from this study was the identification of significant depressive and anxiety symptoms and problem drinking. Additionally, participants could indirectly benefit from knowing that the information provided from the study could assist other caregivers. The research study provided some potential benefits during and after participating in the study. A direct benefit was the screening for depressive, anxiety and hazardous drinking scores. Caregivers with significant depressive, anxiety symptoms, and hazardous drinking scores were given a referral letter to share with their primary health care providers.

Various procedures were implemented to ensure protection of confidentiality throughout the study. All information obtained about the participants in this study, including questionnaires was confidential. However, University of Tennessee Health Center Institutional Review Board had access to confidential data that identified the participant by name in their oversight activities of this research project. Third party insurers or employees did not have access to this research data. The research record did not become a part of the participant's medical record. The research record was labeled with a code number. A master key that linked the name and the code number was 
maintained in a separate locked file cabinet in the PI's research office at the University. The list was destroyed at the end of the study. Data collected during this study were used in reports, presentations, and publications; however, names were not used. Under federal privacy regulation, the participants had the right to determine who had access to their personal health information (called "protected health information" or PHI). The care recipient's PHI collected in this study included the care recipient's medical histories, the results of physical exams, pre and posttests, and other diagnostic and treatment procedures, as well as basic demographic information. No PHI information was gathered on the caregiver only demographic information. The participants were informed that signing the consent form authorized the researchers to have access to care recipient's PHI collected in this study. The PHI was not be used or disclosed to any other person or entity, except as required by law, or for authorized oversight of this research study by other regulatory agencies. Nor was the PHI used for other research except for which the use and disclosure of the PHI was approved by the IRB. The participant's PHI was used only for the research purposes described in the introduction of the study consent form and was used until the study was completed.

The participants were informed that they could cancel authorization in writing at any time by contacting the PI listed on the first page of the consent form. If authorization was canceled, continued use of the care recipient's PHI was permitted if it was obtained before cancellation and its use is necessary in completing the research. However, PHI collected after participant's cancellation was not to be used in the study. Participants refusing to provide this authorization were able to participate in the research study. If the participants canceled the authorization, then they were withdrawn from the study. 
Participants neither withdrew from the study nor cancelled authorization. Finally, federal regulations allow the participants to obtain access to their PHI collected or used in the proposed study. However, in order to complete the study, access to this PHI could be temporarily suspended while the research was in progress. 


\section{CHAPTER 4. RESULTS}

This study had three related purposes. The investigator first described depressive and anxiety symptom levels, prevalence of hazardous drinking, rewards, and subjective burden reported by family caregivers of patients with chronic liver disease (CLD). Second, the compared the depressive and anxiety symptom levels, prevalence of hazardous drinking, subjective burden, and caregiver rewards reported by African Americans and Caucasian family caregivers of patients with CLD. Third, the investigator examined the predictors of subjective burden and mental health status of African American and Caucasian family caregivers of persons with CLD were examined. The initial discussion in this chapter focuses on a description of the sample. The investigator presents the results and statistical analyses of each research question.

\section{Caregiver Characteristics}

Sample characteristics reflect means, standard deviations, frequencies, and percentages unless otherwise stated and summarized in Table 1. The total sample consisted of 73 caregivers. Most caregivers were female (78.1\%) and Caucasian (66\%) while the remaining sample was either African American (31\%) or classified as others (4.1\%). The mean age of study caregivers was 48 years. Forty-one percent of these caregivers worked full time while 19\% were retired and 15\% were homemakers. Six years was the average length of time spent caregiving for this sample of caregivers. 
Table 1. Demographic Characteristics of Caregivers of Individuals with Chronic Liver Disease ( $\mathrm{N}=73)$.

\begin{tabular}{|c|c|c|c|c|}
\hline Characteristics & $\begin{array}{l}\text { Sample } \\
N(\%)\end{array}$ & $\begin{array}{l}\text { African American } \\
n(\%)\end{array}$ & $\begin{array}{l}\text { Caucasian } \\
n(\%)\end{array}$ & $\begin{array}{l}\text { Others } \\
n(\%)\end{array}$ \\
\hline Ethnicity & & $22(30.1)$ & $48(65.8)$ & $3(4.1)$ \\
\hline \multicolumn{5}{|l|}{ Gender } \\
\hline Male & $16(21.9)$ & $9(40.9)$ & 7 (14.6) & $0 \quad(0)$ \\
\hline Female & $57(78.1)$ & $13(59.1)$ & $41(85.4)$ & $3(100)$ \\
\hline \multicolumn{5}{|l|}{ Employment } \\
\hline Full-time & $30(41.1)$ & $9(40.9)$ & 19 (39.6) & $2(66.7)$ \\
\hline Part-time & $5 \quad(6.8)$ & $1(4.5)$ & $4 \quad(8.3)$ & $0(0)$ \\
\hline Unemployed & $11(15.1)$ & $6(27.3)$ & $5(10.4)$ & $0(0)$ \\
\hline Retired & $14(19.1)$ & $3(13.6)$ & $11(22.9)$ & $0(0)$ \\
\hline Homemaker & $10(13.7)$ & $2(9.1)$ & $7(14.6)$ & $1(33.3)$ \\
\hline Student & $2(2.7)$ & 0 & $2(4.2)$ & $0(0)$ \\
\hline Other & $1 \quad(1.4)$ & $1 \quad(4.5)$ & 0 & $0(0)$ \\
\hline \multicolumn{5}{|l|}{$\mathrm{ADL} *$} \\
\hline No assistance needed & $38(52.1)$ & $10(45.5)$ & $27(56.3)$ & $1(33.3)$ \\
\hline Caregiver & $26(35.6)$ & $10(45.5)$ & $15(31.3)$ & $1(33.3)$ \\
\hline $\begin{array}{l}\text { Family or friend } \\
\text { in the household }\end{array}$ & $3 \quad(4.1)$ & $1 \quad(4.5)$ & $2(4.2)$ & \\
\hline $\begin{array}{l}\text { Family or friend } \\
\text { outside the } \\
\text { household }\end{array}$ & $3(4.1)$ & $1 \quad(4.5)$ & $2(4.2)$ & \\
\hline $\begin{array}{l}\text { Professional or } \\
\text { healthcare worker }\end{array}$ & 0 & 0 & 0 & \\
\hline Worried about children & $43(58.9)$ & $15(68.2)$ & $25(52.1)$ & $3(100)$ \\
\hline No & $27(37.0)$ & $7(31.8)$ & $20(41.7)$ & \\
\hline Yes & 1 (1.4) & & $1(2.1)$ & \\
\hline \multicolumn{5}{|l|}{ Not applicable } \\
\hline \multicolumn{5}{|l|}{ Income decrease $^{*}$} \\
\hline No & $52(71.2)$ & $17(77.3)$ & $33(68.8)$ & $2(66.7)$ \\
\hline Yes & $19(26.0)$ & $4(18.2)$ & $14(29.2)$ & $1(33.3)$ \\
\hline
\end{tabular}


Table 1. Cont'd.

\begin{tabular}{lclll}
\hline Characteristics & Sample & $\begin{array}{l}\text { African } \\
\text { American }\end{array}$ & Caucasian & Others \\
& $M \pm S D$ & $M \pm S D$ & $M \pm S D$ & $M \pm S D$ \\
\hline Age (years) & $48.2 \pm 14.7$ & $46.9 \pm 17.2$ & $49.1 \pm 13.6$ & $57.0 \pm 8.9$ \\
Education (years) & $12.4 \pm 2.6$ & $12.5 \pm 2.1$ & $12.4 \pm 2.9$ & $13.3 \pm 1.2$ \\
& $28515.6 \pm$ & $23717.3 \pm$ & $31167.3 \pm$ & $45666.7 \pm$ \\
$\begin{array}{l}\text { Annual Income } \\
\text { (dollars) }\end{array}$ & 21361.2 & 16862.9 & 23265.5 & 13796.1 \\
Years of care & $6.1 \pm 7.2$ & $6.5 \pm 7.3$ & $5.45 \pm 6.3$ & $12.7 \pm 17.0$ \\
& & & & \\
\hline
\end{tabular}

*Note. Table value reflects missing data ADL $n=70$; Income decrease $n=71$; Annual income (dollars) n=61. 
Twenty six percent of study caregivers reported a decrease in income as a result of providing care for their ill relative.

\section{Care Recipients' Characteristics}

Summary data (means, standard deviations, frequencies, and percentages) for care recipients' characteristics are provided in Table 2. The average age of the care recipients was 51 years of age. Care recipients' ethnicity for the study sample was similar to that of the caregiver group; $32 \%$ was African American and 64\% was Caucasian. There were equal numbers of male and female care recipients. Child Pugh Score (CPS) measured liver disease severity and mortality risk (Forman \& Lucey, 2001). Class A was the least severe, while class B was the moderate severe and class C was the most severe. The CPS mean value for participating care recipients was on average the upper limit of class A (6.79 \pm 1.71$)$, the least severe category. As reported in Table 2, the mean score $(7.60 \pm$ SD.72) for care recipients in CPS class B accounted for $41 \%(n=30)$ of the sample, while care recipients in class $C$ the most severe category mean score $(10.3+9.2)$ represented only $11 \%(n=8)$ of the sample. Most of the care recipients were unemployed $(31.5 \%)$ or retired $(26 \%)$. Twenty-eight $(38.4 \%)$ care recipients were diagnosed with cirrhosis, $10(13.7 \%)$ with alcoholic cirrhosis, 27 (37.0\%) with hepatitis C, and 8 (11.0\%) with other diseases or disorders.

\section{Mental Health, Burden, and Reward Scores}

Mean and median scores, standard deviations, and ranges for outcome variables in the total sample of caregivers are provided in Table 3. The caregivers' scores for 
Table 2. Characteristics of Care Recipients Diagnosed with Chronic Liver Disease (N 73).

\begin{tabular}{|c|c|c|}
\hline Characteristics & $\mathrm{M} \pm \mathrm{SD}$ & $n(\%)$ \\
\hline Age (years) & $51.00 \pm 11.53$ & \\
\hline CPS & $6.79 \pm 1.71$ & \\
\hline Class A & $5.34 \pm 0.07$ & $35(48)$ \\
\hline Class B & $7.67 \pm 7.21$ & $30(41)$ \\
\hline Class C & $10.38 \pm 0.51$ & $8(11)$ \\
\hline MELD & $9.95 \pm 3.99$ & \\
\hline \multicolumn{3}{|l|}{ Ethnicity } \\
\hline African American & & $23(31.5)$ \\
\hline Caucasian & & $47(64.4)$ \\
\hline Other & & 3 (4.1) \\
\hline \multicolumn{3}{|l|}{ Gender } \\
\hline Male & & 35 (47.9) \\
\hline Female & & $38(52.1)$ \\
\hline \multicolumn{3}{|l|}{ *Employment } \\
\hline Full-time & & $15(20.5)$ \\
\hline Part-time & & $3(4.1)$ \\
\hline Unemployed & & $23(31.5)$ \\
\hline Retired & & $19(26.0)$ \\
\hline Homemaker & & $7 \quad(9.6)$ \\
\hline Disabled & & $2(5.5)$ \\
\hline Others & & $8(11.0)$ \\
\hline
\end{tabular}

Note. CPS-Child Pugh Score; MELD-Mayo End Stage Liver Disease; *reflects missing data. 
Table 3. Family Caregiver Scores on Mental Health, Burden, and Reward Instruments $(\mathrm{N}=73)$.

\begin{tabular}{|c|c|c|c|c|}
\hline Instrument & $\begin{array}{c}\text { Sample } \\
\mathrm{Mdn} \pm \mathrm{IQR}\end{array}$ & $\begin{array}{c}\text { African } \\
\text { Americans } \\
\text { Mdn } \pm \text { IQR }\end{array}$ & Caucasians & Others \\
\hline CES-D & $17.0 \pm 23.0$ & $17.0 \pm 18.0$ & $20.1 \pm 14.4$ & $12.33 \pm 11.59$ \\
\hline HAM-A & $10 \pm 17.0$ & $12.5 \pm 19.0$ & $11.9 \pm 9.3$ & $10.66 \pm 13.61$ \\
\hline AUDIT & $00 \pm 2$ & $1.17 \pm 2$ & $1.3 \pm 2.9$ & $0.33 \pm 0.57$ \\
\hline \multirow[t]{2}{*}{ Instrument } & Sample & $\begin{array}{c}\text { African } \\
\text { Americans }\end{array}$ & Caucasians & Others \\
\hline & $\mathrm{M} \pm S D$ & $\mathrm{M} \pm S D$ & $\mathrm{M} \pm S D$ & \\
\hline BI & $21.5 \pm 11.9$ & $19.9 \pm 10.7$ & $22.8 \pm 12.4$ & $12.33 \pm 14.43$ \\
\hline PCRS & $52.5 \pm 20.2$ & $61.5 \pm 18.1$ & $47.9 \pm 20.2$ & $58.33 \pm 15.69$ \\
\hline
\end{tabular}

Note. CES-D is the Center for Epidemiological Studies Depression Scale, which measures depressive symptoms; HAM-A refers to the Hamilton Anxiety Rating Scale measures anxiety symptoms; AUDIT is the Alcohol Disorder Use Identification Test measures at risk drinking behavior; BI is the Burden Inventory and measures subjective burden; PCRS refers to the Picot Caregiver Rewards Scale which measures caregiver reward. 
depressive and anxiety symptom levels were right skewed. Scores for subjective burden and rewards were normally distributed. Total scores for CES-D, a measure of depressive symptoms, ranged from 0 (no depressive symptoms) to 47 (severe depressive symptoms). Anxiety scores ranged from 0 (no anxiety) to 42 (severe anxiety). Subjective burden scores ranged from 0 (no burden) to 56 (moderate to severe burden). In this group of caregivers, caregiver reward scores ranged from 0 (no rewards) to 96 (great reward). Total scores for AUDIT ranged from 0 (no alcohol problems) to 16 (medium level of alcohol problems).

\section{Research Aims, Questions, and Findings}

Study findings are reported for each specific aim and question in sequence, with each aim and relevant research questions restated followed by the results of the statistical analyses.

\section{$\underline{\text { Specific Aim One }}$}

Describe depressive symptom levels, anxiety symptom levels, and the prevalence of hazardous drinking, subjective burden, and caregiver rewards in family caregivers of persons with CLD.

\section{Question One}

What are the depressive and anxiety symptom levels, prevalence of hazardous drinking, subjective burden, and caregiver rewards reported by family caregivers of patients with CLD? 


\section{$\underline{\text { Findings }}$}

Data analyses were conducted using Statistical Package for Social Science (SPSS) statistical program. Results are reported as means plus or minus the standard deviation when scores were normally distributed and as median plus or minus the interquartile range if results were non-normal. As shown in Table 3, the median and the interquartile range for depressive symptom levels for the total group was $17.00 \pm 23.00$. Participating caregivers' median scores were greater than the cut off score $(\geq 6)$ for depressive symptoms indicating that this group suffered from mild distress. Twelve caregivers (18\%) had scores of 21-30 indicative of moderate distress from depressive symptoms and 17 caregivers $(23 \%)$ had scores of 31 and higher representing greater than severe distress. African American caregivers' mean score for depressive symptoms was $19.36 \pm 11.43$ and Caucasian caregivers reported a mean score for depressive symptom of $20.08 \pm 14.4$. Sixty percent of the caregivers $(n=44)$ in the current study received referrals to contact a primary care physician because their depressive symptom scores suggested the need for additional evaluation. The median and the interquartile range for levels of anxiety symptoms for the total group was $10.70 \pm 17.0$. Fifty three caregivers $(72 \%)$ reported scores of 0-17 indicative of no anxiety symptoms, 12 caregivers $(16 \%)$ had scores of 18 24 representing mild anxiety symptoms, five caregivers (8\%) had scores of 25-29 representing moderate anxiety, and three caregivers (4\%) had scores of 30 and above representing severe anxiety. Most of the caregivers suffering from mild to severe anxiety were females $(78 \%)$ because most study caregivers were women. Caregivers whose 
scores were 18 and higher received referrals to contact primary care physician because their anxiety symptom scores suggested the need for additional evaluation $(\mathrm{n}=21)$.

The median score and interquartile range for hazardous drinking for the total sample of caregivers was $0 \pm 2.0$, which reflects the prevalence for hazardous drinking (see Table 3). Four caregivers (5\%) in the sample reported significant drinking problem with a score of eight and above. Half of this group of problem drinkers was African American and the other half was Caucasian.

The total sample of caregivers had mild subjective burden (21.5 \pm 11.9$)$. Fifty one percent $(n=37)$ of the total sample of caregivers of persons with CLD reported little or no burden (mean score $0-20)$. Forty four percent $(n=32)$ had mild to moderate burden (mean score 21-40), 5\% $(\mathrm{n}=4)$ had moderate to severe burden $(41-60)$, and none of the caregivers had severe burden (61-88). The total sample of caregivers had a caregiver rewards mean score of $52.5 \pm 20.2$.

\section{$\underline{\text { Specific Aim Two }}$}

Compare the depressive symptom levels, anxiety symptom levels, prevalence of hazardous drinking, subjective burden, and caregiver rewards reported by African American and Caucasian family caregivers of persons with CLD.

\section{Question Two}

What are the differences in level of depressive symptoms, anxiety symptom levels, prevalence of hazardous drinking, subjective burden, and rewards reported by African American and Caucasian family caregivers of patients with CLD? 


\section{$\underline{\text { Findings }}$}

Using the Statistical Package for Social Science (SPSS) statistical program, depressive symptoms, anxiety symptom levels, and hazardous drinking levels were assessed for normality and the distributions were found to be right skewed. Subjective burden and caregiver reward scores for the sample were normally distributed. A Mann Whitney test, one of several nonparametric alternatives to the two sample t-test, was conducted to compare depressive symptoms and anxiety symptom levels and hazardous drinking in African Americans and Caucasian caregivers. The level of depressive symptoms was slightly higher for Caucasians (Mdn17.5 \pm IQR 27.5.) than for African American caregivers (Mdn 17.0 \pm IQR 18.0). However, differences were not statistically significant $(z=-.342, \mathrm{p}=.73)$. Anxiety symptom levels for African American caregivers (Mdn 12.5 \pm IQR 19.0) were slightly higher than Caucasian caregivers' anxiety symptom levels (Mdn 8.0 \pm IQR 17.0) but were not statistically different $(z=-.405, \mathrm{p}=.68)$. Hazardous drinking levels for African American caregivers (Mdn 1.17 \pm IQR 2.0) were similar to Caucasian caregivers' anxiety symptom levels (Mdn 1.3 \pm IQR 2.9) and not statically different $(z=-.514, \mathrm{p}=.60)$. An independent sample t-test was performed to compare mean caregiver burden and caregiver reward scores by ethnic group (African American versus Caucasian caregivers). Caregivers whose ethnicity was listed as "other" were not included in this analysis.

A Levene's test was conducted to determine the equality of variances between the groups. The p-value from the Levene's test indicated that the variance between groups for both subjective burden and reward scores were equal. Therefore the p-value was 
selected from the equal variance table to determine if the differences between the group means were significant. The level of caregiver subjective burden was higher for Caucasians $(23.00 \pm 12.35)$ than the level reported by African Americans (19.95 \pm 10.67$)$. Caucasians reported experiencing more burden than Africans Americans; however, the differences between the means were not statistically significant $(t=-926 ; p=.358)$. Most (83\%) caregivers with mild to severe subjective burden scores in the current study were female. African Americans reported a significantly higher mean score for caregiver rewards $(61.5 \pm 18.07)$ than Caucasians $(47.93 \pm 20.24)$ and these differences were statistically significant $(t=2.68, p=.009)$. On average African American caregivers reported more caregiver rewards than Caucasian caregivers.

\section{$\underline{\text { Specific Aim Three }}$}

Explicate the predictors of mental health status, subjective burden, and caregiver rewards of family caregivers of persons with CLD.

\section{Question Three}

What are the predictors of mental health, subjective burden and caregiver rewards of family caregivers of patients with CLD?

\section{Findings}

Pearson correlation analyses and stepwise multiple regression were used to determine the predictors of subjective burden and mental health of family caregivers of patients with CLD. Before correlation analyses were conducted, the data for categorical 
independent variables were transformed into dummy codes (see Table 4), where the presence of a characteristic was coded 1 and the absence coded 0 . Correlation analyses were performed to determine if there was a relationship between the continuous independent variables (age, years of care) and outcome variables (depressive symptoms, anxiety symptoms, subjective burden, and caregiver rewards). Correlations between other independent variables (ethnicity, gender marital status, relationship to patient, caregivers living arrangement, employment status, income decrease, support or lack of support, and worried about children) and the dependent variables (depressive symptoms, anxiety symptoms, subjective burden and caregiver rewards) were also estimated to investigate the associations among these variables. The independent variables in each domain (caregiver background and contextual factors and primary and secondary stressors) that were significantly associated with each outcome variable were regressed on each identified outcome variable (depressive symptoms, anxiety, subjective burden, and caregiver rewards). Hazardous drinking was omitted from the regression analyses because the caregivers in the current study reported no problems with hazardous drinking.

The magnitude and significance of the linear relationship between independent and dependent variables was used to determine which variables were entered into the regression analyses. Independent variables were entered into the multiple regression models if the bivariate associations between outcome and predictor variables were significant at the .20 . The independent variables, however, were not included in the model if the bivariate relationship between the independent variable and the dependent 
Table 4. Dummy Codes of Caregiver Demographic Variables.

Caregiver Variables Dummy Codes

Ethnicity

Non-African American 0

African American 1

Gender

Female $\quad 0$

Male 1

Marital Status

Not married $\quad 0$

Married 1

Employment Status

Unemployed 0

Employed 1

Relationship to patient

Non-spouse $\quad 0$

Spouse 1

ADL status

Dependent $\quad 0$

Independent 1

Support

Dissatisfied 0

Satisfied 1

Worried about children

No 0

Yes 1 
variable was .65 or greater. This approach was used to reduce the risk of multicollinearity (Burns \& Grove, 2001). Multicollinearity occurs when the independent variables are highly correlated (Schroeder, 1990). Multicollinearity can decrease the power of significance tests and inflates the value of the $b$ coefficient, which will cause it to be significant when in actuality it is not significant (Burns \& Grove, 2001).

No more than four independent variables were chosen for each model to ensure a power of 0.80 with a $p$ value of .05 . In the first model, the caregiver background and contextual domain variables that were significantly correlated with or identified by other investigators as significant correlates of caregiver depressive symptoms (ethnicity, gender, income decrease, and worried about children) were entered into a stepwise regression model with the outcome variable. Following the same principles in the second model, the independent variables from the mediator domain (support), primary and secondary stressor (ADL), worried about children, and income decrease were entered in a model with anxiety level as the dependent variable. In the third model, caregiver income decrease, worried about children, support, and years of care were entered with the outcome variable subjective burden. In the fourth model, the independent variables caregiver ethnicity, relationship with care recipient, employment status, and caregiver age were entered into the stepwise regression model with the dependent variable caregiver rewards. Independent variables were entered or removed from the regression equation by the computer program, based on the amount of additional variance each independent variable contributed to the explained variance of the respective dependent variables (depressive symptoms, anxiety level, subjective burden, and caregiver reward). 
As shown in Table 5, there were several statistically significant bivariate relationships between mental health status, subjective burden and caregiver rewards and demographic variables. Pearson product moment correlations (normally distributed variables) and Spearman correlations (non normally distributed variables) were used to estimate the correlation between dichotomous demographic variables and depressive symptoms, anxiety symptom levels, subjective burden, and caregiver rewards. Caregiver age was negatively correlated with hazardous drinking $(r=-.26 ; p \leq .05)$, years of care was correlated with subjective burden $(r=.40 ; p \leq .01)$, marital status $(r=.27 ; p<.05)$ and ethnicity $(r=.36 ; p \leq .01)$ were correlated with caregiver rewards. Employment status (employed or not employed) was positively correlated with hazardous drinking $(\mathrm{r}=$ $.25 ; \mathrm{p}<.05)$ and negatively correlated with caregiver rewards $(r=-.34, \mathrm{p} \leq .01)$. The variable income decrease was significantly correlated with subjective burden $(r=.40 ; \mathrm{p} \leq$ $.00)$. Support was negatively correlated with level of anxiety $(r=-.26 ; p \leq .05)$ and the variable worried about children was correlated with depressive symptoms $(r=.27 ; \mathrm{p} \leq$ $.03)$ and subjective burden $(r=.42 ; p \leq .00)$. The variable income decrease was significantly related to subjective burden. The variables worried about children were significantly related to depressive symptoms and subjective burden. Caregivers who were worried about their children had more depressive symptoms and subjective burden than caregivers who were not worried about their children. Similarly caregivers who had a decrease in income as a result of caregiving reported more subjective burden than caregivers who reported no decrease in income. Additionally, caregivers with increased support had lower levels of anxiety. 
Table 5. Correlation Analysis between Caregiver Characteristics, Depressive and Anxiety Symptoms, Hazardous Drinking, and Subjective Burden.

\begin{tabular}{lccccc}
\hline & CES-D & HAM-A & AUDIT & BI & PCRS \\
\hline Age & -.072 & -.151 & $-.255^{*}$ & .004 & -.203 \\
$\dagger$ Years of Care & .136 & .185 & .110 & $.255^{*}$ & .067 \\
Marital Status & .029 & .033 & .148 & -.091 & $.235^{*}$ \\
Ethnicity & -.056 & .102 & .043 & -.149 & $.311^{*}$ \\
$\begin{array}{l}\text { Employment } \\
\text { status }\end{array}$ & -.081 & -.061 & $.243^{*}$ & -.037 & $-.323^{* *}$ \\
$\begin{array}{l}\text { Income Decrease } \\
\text { Support }\end{array}$ & $.276^{*}$ & .232 & -.137 & $.397^{* *}$ & -.080 \\
$\begin{array}{l}\text { †Worried } \\
\text { About the } \\
\text { Children }\end{array}$ & -.140 & $-.282^{*}$ & -.135 & $-.257^{*}$ & -.094 \\
$\begin{array}{l}\text { Relationship } \\
\text { To the patient }\end{array}$ & $.271^{*}$ & .192 & -.059 & $.418^{* *}$ & -.083 \\
& & & & & \\
\hline
\end{tabular}

Note: Sample size $=73$ except where noted; Income decrease, $n=71$; Years of care, $\mathrm{n}=66$; Support, $\mathrm{n}=72$; Worried about children, $\mathrm{n}=71 * \mathrm{p} \leq .05, * * \leq 0.00$. 
Utilizing the bivariate results, a series of separate multiple regression analyses using the stepwise procedure was conducted for each of the outcome variables (depressive symptoms, anxiety symptom levels, subjective burden, and caregiver rewards) as previously described. Predictor variables included in the regression analyses were caregiver characteristics (age, ethnicity, gender, employment status), primary stressor (ADL), and mediator variables (social support) that were statistically significant in the respective bivariate analyses. Statistically significant bivariate analyses included variables that depicted weak to strong linear relationships.

The four models that follow display the results of stepwise regression analyses. The first model shows the result (Table 6) of the independent variables (caregiver employment, worried about children, caregiver ethnicity, and caregiver gender) regressed upon depressive symptoms. The independent variable worried about children $(\mathrm{B}=.08, p$ $=.05$ ) accounted for $6 \%$ of the variance in adjusted depressive symptoms; this was the sole variable that remained in the depressive symptom model. Caregivers who were worried about their children had increased depressive symptoms. In the second model, the independent variables (caregiver support, ethnicity, caregiver gender, and assistance with ADL) were regressed upon anxiety symptom levels. None of these predictor variables — caregiver support, ethnicity, caregiver gender, or assistance with ADL—was a statistically significant predictor of anxiety symptom levels. As shown in Table 7, the third model consisted of the independent variables (caregiver income decrease, worried about children, support, and years of care) regressed upon subjective burden. The variables Income Decrease $(\mathrm{B}=.353, p=.003)$ and Worried about Children $(\mathrm{B}=.349, p$ 
Table 6. Stepwise Multiple Regression Analysis of Depressive Symptoms and Caregiver Gender, Caregiver Ethnicity, Caregiver Employment, and Worried about Children $(n=66)$.

\begin{tabular}{ccc}
\hline Independent Variables & Beta & $p$ \\
\hline Worried about Children & .267 & .03 \\
\hline
\end{tabular}

Note. Adj $\mathrm{R}^{2}=.056, \mathrm{~F}=5.08 \mathrm{df}=1,62, \mathrm{p}=.03$ (model statistics); Excluded variables: caregiver gender, ethnicity, and caregiver employment.

Table 7. Stepwise Multiple Regression Analysis of Subjective Burden and Caregiver Income Decrease, Worried about Children, Support, and Years of Care $(n=62)$.

\begin{tabular}{lll}
\hline Independent Variable & Beta & $p$ \\
\hline Income Decrease & .353 & .003 \\
Worried about Children & .349 & .003 \\
\hline
\end{tabular}

Note. Adj $\mathrm{R}^{2}=.255, \mathrm{~F}=10.91 \mathrm{df}=2,56 \mathrm{p}=\leq .000$ (model statistics); Excluded variables: support and years of care. 
$=.003$ ) remained in the final model as predictors of subjective burden accounting for $26 \%$ of adjusted variance.

Caregivers who reported a decrease in income and worried about their children reported greater subjective burden. The independent variables (caregiver ethnicity, caregiver relationship, caregiver employment status, and caregiver marital status) were regressed upon caregiver rewards (Table 8). Caregiver ethnicity $(\mathrm{B}=.37, p=.001)$ and employment status $(\mathrm{B}=-.33, p=.003)$, remained in the final regression model as predictors of caregiver rewards accounting for $22 \%$ of the variance. Being African American and unemployed was predictive of increased caregiver rewards in the current study.

Mulitcollinearity within the regression models was assessed using tolerance, variance inflation factors, and condition index. Examination of these estimates for each predictor variable revealed the validity of each regression model (Tables 9-12). If the tolerance of all predictor variables is 1 there is no correlation. The tolerance in the current study models ranged from .8 to .9 indicating that the predictor variables were not correlated. When there is no multicollinearity between the predictor variables the variance inflation factors is also 1.0 and should not exceed 10 (Schroeder, 1990). The variance inflation factors for the predictor variables in the current study models ranged from 1.0 to 1.1 , whereas the condition indexes ranged from 1.0 to 9.3. Condition index values less than 30 indicate the absence of multicollinearity. 
Table 8. Stepwise Multiple Regression Analysis of Caregiver Reward and Caregiver Ethnicity, Caregiver Relationship, Employment, and Marital Status $(\mathrm{n}=69)$

\begin{tabular}{lcc}
\hline Independent Variable & Beta & $p$ \\
\hline Caregiver Ethnicity & .373 & .001 \\
Caregiver Employment & -.330 & .003 \\
\hline
\end{tabular}

Note. Adj $\mathrm{R}^{2}=.222, \mathrm{~F}=10.70 \mathrm{df}=2,66, \mathrm{p}=\leq .000$ (model statistics); Excluded variables: caregiver relationship and marital status.

Table 9. Collinearity Diagnostics for the Model of Depressive Symptoms ( $n=64)$.

\begin{tabular}{lllrl}
\hline Variable & df & Tolerance & $\begin{array}{l}\text { Variance } \\
\text { Inflation }\end{array}$ & $\begin{array}{l}\text { Condition } \\
\text { Index }\end{array}$ \\
\hline Caregiver Gender & 1 & 0.993 & 1.007 & 1.000 \\
Caregiver Ethnicity & 1 & 0.984 & 1.016 & 1.000 \\
Income Decrease & 1 & 0.990 & 1.010 & 1.000 \\
Worried about & 1 & 1.000 & 1.000 & 2.091 \\
Children & & & & \\
\hline
\end{tabular}

Table 10. Collinearity Diagnostics for the Model of Anxiety Symptom Levels $(n=58)$.

\begin{tabular}{llllc}
\hline Variable & df & Tolerance & $\begin{array}{c}\text { Variance } \\
\text { Inflation }\end{array}$ & $\begin{array}{c}\text { Condition } \\
\text { Index }\end{array}$ \\
\hline Caregiver Support & 1 & 0.958 & 1.004 & 2.147 \\
Activities of Daily Living & 1 & 0.928 & 1.077 & 2.566 \\
Income Decrease & 1 & 0.945 & 1.058 & 2.977 \\
Worried about Children & 1 & 0.943 & 1.061 & 7.507 \\
\hline
\end{tabular}


Table 11. Collinearity Diagnostics for the Model of Subjective Burden Levels $(n=66)$.

\begin{tabular}{lllll}
\hline Variable & df & Tolerance & $\begin{array}{c}\text { Variance } \\
\text { Inflation }\end{array}$ & $\begin{array}{c}\text { Condition } \\
\text { Index }\end{array}$ \\
\hline Income Decrease & 1 & 0.978 & 1.022 & 1.827 \\
Worried about Children & 1 & 0.978 & 1.022 & 1.000 \\
Caregiver Support & 1 & 0.933 & 1.072 & 1.893 \\
Years of Care & 1 & 0.927 & 1.078 & 2.406 \\
\hline
\end{tabular}

Table 12. Collinearity Diagnostics for the Model of Caregiver Rewards $(n=72)$.

\begin{tabular}{lllll}
\hline Variable & df & Tolerance & $\begin{array}{l}\text { Variance } \\
\text { Inflation }\end{array}$ & $\begin{array}{c}\text { Condition } \\
\text { Index }\end{array}$ \\
\hline Caregiver Ethnicity & 1 & 1.000 & 1.000 & 1.000 \\
Caregiver Relationship & 1 & 0.918 & 1.089 & 1.827 \\
Caregiver Employment & 1 & 1.000 & 1.022 & 1.967 \\
Marital Status & 1 & 0.820 & 1.096 & 2.866 \\
\hline
\end{tabular}




\section{CHAPTER 5. DISCUSSION AND IMPLICATIONS}

\section{Discussion}

This study investigated depressive symptoms, anxiety symptom levels, hazardous drinking, subjective burden, and caregiver rewards in African Americans and Caucasian caregivers of patients with CLD. Study findings also provided information related to factors that predicted mental health outcomes, subjective burden, and caregiver rewards in the study sample. This chapter includes an expanded discussion of significant findings as well as an appraisal of the findings in light of existing research studies. Limitations of the study and implications for theory, research, and practice are also discussed, particularly directions for interventions to reduce deteriorating mental health and subjective burden.

\section{$\underline{\text { Demographic Findings }}$}

Many of the study findings are consistent with published literature while some findings were not. There were more females in the study than males, which is consistent with the general caregiving literature indicating that females function as caregivers more often than males (Belasco \& Sesso, 2002; Brazil, Bedard, Willison, \& Hode, 2003; Cain \& Wicks, 2000; McCullagh et al., 2005; Pinquart \& Sorensen, 2006). Caregivers in the current study were younger, 48 years of age, when compared to other research studies where the caregivers were on average 55 and older (Brouwer et al., 2004; Chio, Guathier, Calvo, Ghiglione, \& Mutani, 2005; Hecht et al., 2003; McCullagh et al., 2005; NavaieWaliser et al., 2002). The possible reason for this difference in age is that CLD and 
cirrhosis affects individuals at a younger age than other chronic illnesses such as Alzheimer's disease. Most caregivers were spouses (45\%) and children (21\%), who typically are around the same age (spouses) or younger (children) than persons with the disorder. Like other research studies focusing on the caregiving experience, most of the caregivers were employed full-time (Belasco \& Sesso, 2002; Croog et al., 2001; Harris, Thomas, Wicks, Faulkner, \& Hathaway, 2000; Navaie-Waliser et al., 2002; Teel, Duncan, \& Lai, 2001). Additionally, most (53\%) caregivers in the study were married, $52 \%$ reported that patients did not need assistance with ADL, 36\% assisted the care recipient with ADL, and $74 \%$ lived with the care recipient. In the current study, ethnic minorities represented $30 \%$ of the sample. Many studies include insufficient numbers of ethnic minority caregivers (Dilworth-Anderson et al., 2002).

Patients that these caregivers assisted included persons with hepatitis $\mathrm{C}$, which represented $37 \%$ of the patients in the study sample. Individuals with cirrhosis represented $43 \%$ of the total sample. Both Caucasian (36\%) and African American (38\%) patients had similar rates of hepatitis C. These rates are dissimilar to the general population. The prevalence of African Americans with hepatitis C in the U.S. is 3.2\% while approximately $22 \%$ of African Americans are diagnosed with cirrhosis compared to $30 \%$ of Caucasians. The progression rate of hepatitis $\mathrm{C}$ toward cirrhosis is slower in African Americans when compared to Caucasians and is not well understood (Pyrsopoulos \& Jeffers, 2005). This high rate of both diseases in the African American study participants probably reflects the fact that this was a select group seeking treatment. A sample derived from a non-help seeking population would likely include fewer African Americans. A second plausible explanation for this increased rate among African 
Americans is that this ethnic group represents $63 \%$ of the Memphis and Shelby County population (U.S. Census, 2000). Finally, there may have been differences in the socioeconomic status by ethnicity with African Americans having fewer economic resources; hepatitis $\mathrm{C}$ is more common in poor populations. The National Health and Nutrition Examination Survey (NHANES) 1988-1994 reported 5.7\% prevalence of hepatitis $\mathrm{C}$ in the United States in ethnic minorities below the poverty index compared to 1.9 at or above the poverty index (Kruszon-Moran and McQuillan, 2005).

\section{Mental Health Symptoms and Hazardous Drinking}

The first research aim of this study focused on describing depressive symptom levels, anxiety symptom levels, and the prevalence of hazardous drinking in family caregivers of persons with CLD. Many studies document increased depressive symptoms among family caregivers (Croog et al., 2001; Given et al., 2004; Pinquart \& Sorensen, 2004; Pirraglia et al., 2005; Yee \& Schultz, 2000). Depressive symptom scores for the study sample exceeded the cut-off of 16 on the CES-D placing this group of caregivers in the category of experiencing at least mild distress. African American caregivers were slightly less depressed when compared to Caucasians but these differences were not statistically significant. Study results are consistent with the literature reporting that African American caregivers experience less depression compared to Caucasian caregivers (Covinsky et al., 2003; Dilworth-Anderson et al., 2002; Haley et al., 1995; Janevic \& Connell, 2001; Knight et al., 2000; Miller, Campbell, Farran, Kaufman, \& Davis, 1995; White et al., 2000). Moreover, African Americans express symptoms of depression often report more somatic complaints than Caucasians (Brown, Schulberg, \& 
Madonia, 1996b; Brown, Schulberg, Sacco, Perel, \& Houck, 1999b; Harman, Edlund, \& Fortney, 2004). The CES-D does not conceptually distinguish between somatic complaints and depressive affects (Nguyen, Kitner-Triolo, Evans, \& Zonderman, 2004). Another scale such as the Beck Depression Scale that has a greater focus on somatic symptoms may be more relevant for African American populations. Despite this potential methodological limitation, the average CES-D score indicated that significant numbers of both African American and Caucasian caregivers in this study reported clinically important levels depressive symptoms that warranted follow-up.

\section{$\underline{\text { Anxiety Symptom Levels }}$}

The caregivers in the current study reported low levels of anxiety on the HAM-A scale $(12.67 \pm 10.28)$. A score of 18 and higher was indicative of mild to severe anxiety symptom levels. Previous research studies have investigated the link between the caregiving experience and anxiety with varied results (Dura, Stukenberg, \& KiecoltGlaser, 1991; Garand, Dew, Eazor, DeKosky, \& Reynolds, 2005; Knight, Silvestein, McCallum \& Fox, 2000; Vanderwerker, Laff, Kadan-Lottick, McColl, \& Prigerson, 2005; Yee \& Schultz, 2000). These varied results likely reflect the use of different instruments across caregiver studies. Caregivers assisting family members with dementia reported mid-range levels of anxiety (M $42.45 \pm 9.8$ ) as measured by the Spielberger State Trait Anxiety Scale (STAI) while relatively low levels (29.40 \pm 8.42$)$ were reported by caregiver spouses assisting relatives with mild cognitive impairment (Garand, Dew, Eazor, DeKosky, \& Reynolds, 2005). Scores on the STAI range from 20-80 with higher scores indicating more anxiety measured by the (Knight, Silvestein, McCallum, \& Fox, 
2000). In the current study, $78 \%$ of the caregivers suffering from mild to severe anxiety were females compared to $22 \%$ male. Findings from several studies indicate that female caregivers experience more anxiety than males (Croog et al., 2001; Garand et al., 2005; Yee \& Schultz, 2000); the current study did not examine gender differences in anxiety. In study conducted by Croog et al, (2001), anxiety levels were significantly higher in men than women were in a study investigating husband and wife caregivers of patients diagnosed with Alzheimer disease. The males in the current study were younger (44.2+ 16.5 years) than caregivers in the Croog et al study, who on average were 68 years of age. Additionally, only five males experienced mild to severe anxiety in the current study. The younger age of men participating in the current study may account for the mild to severe anxiety symptoms reported. Older men may be more anxious compared to younger men about the caregiver role, as they likely were not socialized to take on the caring role. The fact that significantly more women participated in the current study likely explains the greater prevalence of mild to severe anxiety in women.

The results of the AUDIT revealed there was no problem drinking in either African American or Caucasian caregivers in the study sample. Few researchers have investigated alcohol use in family caregivers. However, two studies that focused on alcohol use in caregivers found no significant difference in alcohol use in caregivers when compared to non-caregivers population (Cochrane, Goering, \& Rogers, 1997; Polen \& Green, 2001). Similar to previous studies, $5 \%$ of caregivers in the current study had AUDIT scores of eight and above. These scores may not reflect true prevalence of caregivers with a drinking problem because individuals with significant problems may deny the actual amount of alcohol that they consumed (Dawson, 2003). 


\section{$\underline{\text { Subjective Burden and Caregiver Rewards }}$}

Caregivers in the current study on average reported mild subjective burden $(21.5$ $\pm 11.9)$. This is the first research study focusing on caregivers of individuals diagnosed with CLD. Investigators have found dissimilar results in the other caregiving populations. In studies investigating caregivers of patients with Alzheimer's disease, amyotrophic lateral sclerosis, strokes, arthritis and cancer, caregivers reported high levels of caregiver burden related to the caregiving experience (Andrews, 2001; Chio, Gauthier, Calvo, Ghiglione, \& Mutani, 2005; Donelan et al., 2002; Jacobi et al. 2003; McCullagh, Brigstocke, Donaldson, \& Kalra, 2005; Teel, Duncan, \& Lai). Most of the caregivers with mild to severe subjective burden scores in the current study were females (83\%) compared to males $(17 \%)$. Other researchers also reported that reported female caregivers report higher levels of subjective burden than males (Gallicchio, Siddiqi, Langenberg, \& Baumgarten, 2002; Given et al., 2004; Navaie-Waliser et al., 2002; Pinquart \& Sorensen, 2006; Sarna \& Chang, 2000; Tsai \& Jirovec, 2005; Yee \& Schultz, 2000). The mean age for caregivers experiencing mild to severe burden in the current study was $51.27 \pm 9.92$ years. Other investigators report that younger caregivers report more burden compared to older caregivers (Cain \& Wicks, 2000; Vitaliano, Russo, Young, Becker, \& Maiuro, 1991; Zarit et al., 1986). Caregivers in the current study were relatively young compared to other studies. Many factors contribute to the perception of burden. Forty-two percent of the caregivers in the current study with mild to severe burden worked full time, $17 \%$ were homemakers, $11 \%$ unemployed, and $6 \%$ worked part-time. Forty-three percent of these caregivers reported that the care recipient did not 
need assistance with ADL; however, $46 \%$ reported that they provided assistance with ADL. Caregivers in the current study experiences of subjective burden could reflect the influence of age, gender, employment status, and assistance with ADL; the investigator will examine this influence in future studies.

Caregivers of persons with CLD who provided more years of care to their relative had greater levels of subjective burden. Previous research examining the relationship between length of care and subjective burden is equivocal. Some researchers report that more years of caregiving is related to greater burden (Brazil et al., 2003; Brouwer et al., 2004; Rivera-Navarro, Morales-Gonazlez, Benito-Leon, \& GEDMA, 2003) while other researchers have not found a significant association between the two variables (Pinquart \& Sorensen, 2006; Seltzer \& Wailing, 1996). Perhaps caregivers' knowledge of the prognosis and trajectory of their relative's disease increased worry and subjective burden over time. Chronic liver disease is associated with significant disability over time and in severe disease may progress to death. Hepatitis $\mathrm{C}$ progresses to cirrhosis in $20 \%$ of patients and some patients will progress to end-stage liver disease over a span of 20 years (Moylan \& Muir, 2005; Patel et al., 2006). There is also significant social stigma associated with alcohol misuse and thus alcohol-related liver disease; this stigma could have contributed to burden over time in this population as well given that $13 \%$ of care recipients in this study experienced alcohol-related disease.

The mean score for caregiver rewards for the total sample was $52.5 \pm 20.2$. Scores on the PCRS ranged from 0-100. Higher scores indicate higher caregiver rewards. Education is inversely related to caregiver rewards. Caregivers with higher educational attainment perceive less caregiver rewards compared to caregivers with less education 
(Picot, Debanne, Namazi, \& Wykle, 1997; Roff et al., 2004). The mean education of caregivers in the current study was $12.4 \pm 2.6$ years compared to $13.42 \pm 2.53$ years in Picot et al. study. However, African Americans report a higher level of rewards compared to Caucasian caregivers; $30 \%$ of caregivers in this sample was African American (Cohen, Colantonio \& Vernich, L. 2002; Picot, 1995 Picot, Debanne, Namazi, \& Wykle, 1997). The relatively high educational level of caregivers in concert with the significant number of African American caregivers participating in the current study probably explains the overall moderate levels of rewards reported in the study sample.

$\underline{\text { Ethnic Differences in Subjective Burden and Caregiver Rewards }}$

The second aim of the study was to compare the subjective burden and caregiver rewards reported by African American and Caucasian family caregivers of persons with CLD. African American caregivers experience little or no subjective burden compared to Caucasians mild to moderate subjective burden. These results however were not significantly different. Researchers conducting previous studies report Caucasians oftentimes experience more burden than African Americans (Dilworth-Anderson et al., 2002; Janevic \& Connell, 2001; Knight et al., 2000; Roth et al., 2001; White et al., 2000). The differences in the two groups could reflect cultural variations in the perception of the caregiving role. Perceptions of family obligations, role expectations and beliefs about aging influence the subjective burden experienced by African Americans (Dilworth-Anderson et al., 2002; Janevic \& Connell, 2001; White et al., 2000). Socioeconomic status (SES) may have influenced caregivers' perceptions of subjective burden, as none of the research studies controlled for SES. Factors often 
attributed to ethnicity and race could be due to differences in resources (White et al., 2000).

Similarly, our results are consistent with published research indicating that African American caregivers report significantly greater rewards when compared to Caucasians (Picot, 1995; Picot et al., 1997). Although the sample for the current study was relatively small results are likely valid because of they are consistent with published findings.

The study's results support aspects of the Stress Process Model indicating that attributes such as ethnicity significantly influence the caregiving experience and the intensity of stresses. Caregiver characteristics influence the caregivers' expression of stress and the ability to use personal and social resources (Pearlin et al., 1990). In several published studies, perceptions of caregiver reward consistently vary by caregiver ethnicity. Researchers have found a correlation between spirituality and caregiver rewards in African American caregivers reporting prayer and spiritual practices as a mode of coping (Picot, 1995; Picot et al., 1997; Roff et al., 2004; Spurlock, 2005). The cultural importance of spirituality in African American populations may explain variations in reports of rewards associated with the caregiver role.

Another important association in the study was the significant relationship between marital status and caregiver rewards. Married caregivers reported more caregiver rewards than unmarried caregivers did. These results are consistent with the literature indicating that spouses are more likely to perceive greater reward when caring for a chronically ill partner (Savage \& Bailey, 2004; Wallsten, 2000a). 


\section{$\underline{\text { Predictors of Mental Health and Burden }}$}

The last aim explicated the predictors of subjective burden and mental health status of family caregivers of persons with CLD. In this study, as depressive and anxiety symptoms and problem drinking reflected mental health. There are few published studies that investigate problem drinking in caregivers and no studies investigating caregivers of family members with liver disease. Young adults are often identified as the high-risk population for alcohol use (National Household Survey on Drug Abuse (NHSDA), 2001); some caregivers in this study were young adults and they were on average younger than caregivers participating in many published studies were. In the U.S., alcohol misuse accounts for $20 \%$ of liver disease (Diehl, 2002; Menon et al., 2001); 13\% of care recipients in the study had CLD due to alcohol misuse. Alcoholism occurs in families (Anda et al., 2002; Hurcom et al., 2000); therefore, some caregivers likely share similar ineffective coping strategies with the care recipient and consequently use alcohol when significantly stressed, or depressed. Employed caregivers in this study also reported more problem drinking and less caregiver rewards. Caregivers who are employed and caring for an ill relative are may suffer from difficulty balancing the responsibilities of work and caregiving.

Having an income decrease because of caregiving was correlated with increased depressive symptoms, anxiety levels, and subjective burden in the study sample. Caregivers with a low income report insurmountable challenges (Grunfeld et al., 2004; Jacobi et al., 2003; Williams et al., 2003), however these studies focus on current income rather than if the caregiver has had a decrease in income as a result of the caregiving role. Losing financial resources probably increases the challenge that these caregivers face. 
Caregivers with more support reported less anxiety and subjective burden. The results from previous research in this area are mixed. Some investigators report that social support did not affect burden or caregiver mental health outcomes (Choo et al., 2003; McNally et al., 1999; Tsai \& Jirovec, 2005). In contrast, other researchers report that support mediates caregiver burden and caregivers who receive support often report less burden and strain (Clyburn et al., 2000; Nijboer et al., 1999).

Consistent predictors of depressive symptoms and subjective burden were worried about children and income decrease. Caregivers who were worried about their children had higher levels of depressive symptoms than those who were not worried about their children. Parental responsibilities for children in addition to caregiving responsibilities that caused additional challenges may account for these significant relationships. To our knowledge, this is the first study that explored worries about children within the context

of family caregiver. This variable seems particularly important given that these caregivers were relatively young compared to caregivers in other studies.

\section{Limitations and Strengths}

The study has several limitations as well as strengths. Limitations of the study include sampling strategies, response rate, and instrumentation. Strengths include the investigation of new population of caregivers, the examination of several mental health outcomes, and the influence of income decrease on caregivers of persons with CLD. Limitations are discussed first, followed by a discussion of the study strengths.

Participating caregivers lived in metropolitan and southern rural areas, thus it is inappropriate to generalize the study's results to caregivers who live in other geographic 
regions or who differ with regard to demographic characteristics. Caregivers in this study were primarily female, white, high school educated, married, and employed full time.

This study descriptive correlation study design included a non-probability sample. Convenience sampling limits the external validity of a study. Additionally, half of the eligible caregivers did not return completed questionnaires. Response rates of less than $40 \%$ are common studies using mail-in questionnaires (Kerlinger \& Lee, 2000; Sitzia \& Wood, 1998). The 56\% response rate that occurred in this study may reflect a sub sample; therefore, placing the study at risk for sampling bias which poses a threat to internal and external validity. Low response rates mask statistically significant relationships that truly exist within the population studied (Edwards et al., 2002; Halpern, Ubel, Berlin, \& Asch, 2002). Caregivers who were severely depressed, anxious, and or burdened were probably not represented in the study sample.

Instrumentation was also a study limitation. The instruments used in this study were self-assessment tools, which may not reflect the true experience of the caregivers; however, the goal of the study was to assess perceptions of most outcomes. Response error may have occurred when the caregivers' in this study completed the AUDIT questionnaire. When investigators ask participants for sensitive information related to alcohol consumption, they may intentionally misreport information or have trouble accurately recalling alcohol consumption (Dawson, 2003). Additionally, individuals who have problematic drinking behavior often have a propensity to minimize the amount of alcohol consumption consumed (Sjoberg, 1998; Tucker \& Vuchinich, 2000).

Despite the limitations, the present study has several strengths. There are currently no published research studies examining the experiences of family caregivers of 
persons with CLD despite the fact that some chronic liver diseases have debilitating, lifethreatening symptoms. More severe symptoms have been associated with increased caregiver subjective burden and depressive symptoms in other patient populations (Andrews, 2001; Kozachik et al., 2001). Caregivers of patients with a class B or C Child Pugh Scores had higher depressive symptom scores than caregivers assisting patients with class A ratings. Chronic liver disease is the $10^{\text {th }}$ leading cause of death in the United States affecting 25, 000 Americans per year (Riley \& Bhatti, 2001). Hepatitis C affects 4 million people in the United States causing 12,000 deaths per year. Hepatitis C is the leading cause of cirrhosis and has a 40\% morbidity rate (Clark et al., 2002; Daniel, 2005; Koff, 1998). Twenty-eight percent of the 73 patients in this study had hepatitis $\mathrm{C}$ and $28 \%$ had cirrhosis as a complication of hepatitis $\mathrm{C}$. These caregivers and their ill relatives were faced with a potentially life-threatening disease. Some aspects of caregiving include anticipating medical appointments, ensuring family members are compliant with medication, and assisting family members in making healthcare decisions (Ekwall et al., 2004). Caregivers in this study likely participated in each of these caregiving endeavors.

The fact that few studies investigate anxiety and alcohol use in family caregiver populations is strength of this study as both variables were examined in the current study. Caregivers of persons with liver disease have the potential of using ineffective coping. The caregivers in the present study did not report significant problem drinking. Researchers in several studies found consistent results that there were no differences in alcohol use when comparing caregivers with noncaregivers (Cochrane et al., 1997; Mort, Gasper, Juffer, \& Kovarna, 1996; Polen \& Green, 2001). 
Few studies have examined the relationship between income and mental health symptoms within the context of caregiving. In the present study, income decrease consistently predicted increased depressive symptoms and subjective burden. Other investigators have simply looked at income at the time of data collection rather than a decrease in income and its impact on the experience (Williams et al. 2003), which may be more important. Care recipients often become disabled. When care recipients are men and the primary breadwinners, this reduction in income likely creates significant financial hardship.

\section{Practice and Policy Implications}

In this section, implications for practice and policy are discussed. Implications for practice reflect the study's findings related to cultural differences in African American and Caucasian's perceptions of caregiving. Similar to previous research, the results from this study indicate that African Americans report less burden and more caregiver reward compared to Caucasians. African Americans have a distinct culture with variations like other ethnic groups and these variations likely influence all aspect of their lives including the caregiving experience (Dilworth-Anderson, Williams, \& Gibson, 2002). Practitioners should be aware of these differences and their potential implications. Some investigators report that caregiver rewards are mediators between caregiver burden and negative mental health outcomes (Cohen, Colantonio, \& Vernich, 2002; Son, Zauszniewski, Wykle, \& Picot, 2000). Caregivers who can identify rewarding aspects of caregiving are less likely to suffer depression or other negative mental health outcomes (Cohen, Colantonio, \& Vernich, 2002). Other researchers report that perhaps caregiver reward is a 
separate phenomenon and can exist in the same context as increased burden (Andren \& Elmstahl, 2005; Brouwer, van Exel, van den Berg, van den Bos, \& Koopmanschap, 2005). Therefore, clinicians should be aware that caregivers could experience rewards and burden in the same context. Clinicians should assess for both burden and rewards as well as explore mental health outcomes in these individuals. Additionally, African Americans may not score high on the burden scale because of resilience that is often seen because of lifelong challenges such as poverty and racism. Coping with these stressors may have given some of these caregivers' effective coping skills to handle stressful situations such as chronic caregiving. Moreover, spirituality is an important coping strategy in many African American communities (Dilworth-Anderson, Williams, \& Gibson, 2002), which could moderate life stressors like chronic caregiving. Therefore, scores on the burden and caregiver reward scales should be examined in context of cultural influence. Practitioners should consider family caregivers as they treat African American patients with chronic conditions. African American caregivers may perceive the experience as rewarding while simultaneously experiencing significant burden.

Decrease in income and worries about their children were predictors of subjective burden and depressive symptoms; interventions targeting these predictors could be beneficial for this population of caregivers. Programs that offer incentives to caregivers and their families to offset the income decrease caused by the caregiving experience should be implemented. Caregivers often experience loss in work hours, which results in loss of income when providing care for a family member (Grunfeld et al., 2004). A state imposed monetary incentive similar to the program funded by Robert Wood Johnson Foundation could be beneficial to offset the impact of loss of income. Arkansas was one 
of the three states participating in this program (Polivka, 2001), which provides financial monthly cash allowances to eligible participants. Eight out of ten caregivers who received these incentives reported that the allowance had improved their lives. Cash incentives could be provided as an allowance and recipients allowed to spend cash as needed. For example, for some caregivers prescription drugs expenditures accounted for a significant portion of the financial burden reported by caregivers (Grunfeld et al., 2004).

\section{Theoretical Implications}

The study's results support aspects of the Stress Process Model indicating that attributes such as ethnicity significantly influence the caregiving experience and the intensity of stresses. Caregiver characteristics influence the caregivers' expression of stress and the ability to use personal and social resources (Pearlin et al., 1990). In several published studies, perceptions of caregiver reward consistently vary by caregiver ethnicity. Researchers have found a correlation between spirituality and caregiver rewards in African American caregivers reporting prayer and spiritual practices as a mode of coping (Picot, 1995; Picot et al., 1997; Roff et al., 2004; Spurlock, 2005). The cultural importance of spirituality in African American populations may explain variations in reports of rewards associated with the caregiver role. Study findings provided no confirmation for the proposed conceptual model (Figure 2) that predicts the relationships among primary and secondary stressors, caregiver background and contextual factors, mediators, and caregiver outcomes in caregivers of patients with CLD. 


\section{Recommendations for Further Research}

In the current study, the variable worried about children was a significant predictor of depressive symptoms and subjective burden. Published studies do not carefully examine caregiving experiences in the context of child rearing responsibilities. Information about specific worries related to children was not the focus of this study and deserves more focus in future studies. This variable is a previously untested predictor not explored in other caregiving studies. Worried about children were probably important for this sample of caregivers because they were relatively young and typically had childrearing responsibilities, unlike older caregivers often depicted in published studies. A focus group could be conducted to obtain information specifically related to caregivers' worries about their children so that appropriate strategies related to this predictor of caregiver outcomes can be effectively addressed. Perhaps cognitive behavioral strategies could be taught to strengthen the coping skills of these caregivers. This type of broad approach could be applied across areas of concern, including worries about children. Moreover, cognitive behavioral approaches might reduce depressive symptoms in these caregivers as they have been shown to reduce depression in women (Peden, Rayens, Hall, \& Grant, 2005)

The results from previous research are conflicting related to the effects of social support on subjective burden and mental health of caregivers. Some researchers report social support is effective in decreasing distress in family caregivers (Choo et al., 2003; Clyburn, Stones, Hadjistavropoulos, \& Tuokko, 2000; Haley, LaMonde, Han, Burton, \& Schonwetter, 2003) while other research states social support is not effective in decreasing burden and negative mental health outcomes (Choo et al., 2003; Hecht et al., 
2003). In the current study, social support was negatively associated with anxiety levels. Caregivers with receiving less support reported more anxiety. Research is needed seeking information from caregivers related to the perception of useful resources. There are reports that African American caregivers differ in their perceptions of useful resources. Group interventions appear to be most effective in African American and non white ethnic groups (Yin, Zhou, \& Bashford, 2002).

\section{Summary}

Caregiving is a complex phenomenon. Many studies investigate caregivers of persons with other chronic diseases however; researchers have not investigated caregivers assisting a relative diagnosed with liver disease. This study provided partial support for the association among caregiver characteristics and mental health outcomes and therefore provides partial support for the SPM. Study findings emphasize the importance of caregivers' mental health as more than half caregivers in the study reported clinically important levels of depressive symptoms. Predictors of depressive symptoms, subjective burden, and caregiver rewards varied but included worried about children, income decrease, ethnicity, and employment. Information related to these predictors will assist health care providers to identify caregivers at risk for poor mental health outcomes. This study highlights the caregiver characteristics that are related an increased of depressive symptoms and subjective burden and predictive of caregiver reward. Although participants in the current study included caregivers of persons diagnosed with CLD, the

study findings are similar to those found in other caregiver populations. Previously tested interventions that improve these outcomes may be relevant to this population. 


\section{LIST OF REFERENCES}

Acton, G. (2002). Health-promoting self-care in family caregivers. Western Journal of Nursing Research, 24(1), 73-66.

Acton, G., \& Kang, J. (2001). Interventions to reduce the burden of caregiving for an adult with dementia: A meta-analysis. Research in Nursing and Health, 24, 349360.

Aguglia, E., Onor, M., Trevisiol, M., Negro, C.S., M., \& Maso, E. (2004). Stress in caregivers of Alzheimer's patients: An experimental investigation. American Journal of Alzheimers Disorders and other Dementias, 19(4), 248-252.

Amirkhanyan, A., \& Wolf, D. (2003). Caregiver stress and noncaregiver stress: Exploring the pathways of psychiatric morbidity. The Gerontologist, 43(6), 817827.

Anda, R., Whitfield, C., Felitti, V., Chapman, D., Edwards, V., Dube, S., \& Williamson, D. (2002). Adverse childhood experiences alcoholic parents, and later risk of alcoholism and depression. Psychiatric Services, 53(8), 1001-1009.

Andren, S., \& Elmstahl, S. (2005). Family caregivers' subjective experiences of satisfaction in dementia care: Aspects of burden, subjective health and sense of coherence. Scandinavian Journal of Caring Science, 19, 157-168.

Andrews, S. (2001). Caregiver burden and symptom distress in people with cancer receiving hospice care. Oncology Nursing Forum, 28(9), 1469-1474.

Annerstedt, L., Elmstahl, S., Ingvad, B., \& Samuelsson, S. (2000). An analysis of the caregiver's burden and the "breaking point" when home care becomes adequate. Scandinavian Journal of Caring Science, 28, 23-31.

Armstrong, G., Wasley, A., Simard, E., McQuillan, G., Kuhnert, W., \& Walter, M. (2006). The prevalence of hepatitis C virus infection in the United States, 1999 through 2002. Annals of Internal Medicine, 144(10), 705-714.

Babor, T., De la Fuente, J., Saunders, J., \& Grant, M. (1992). AUDIT the alcohol use disorders identification test guidelines for use in primary health care. World Health Organization. WHO Press, Geneva Switzerland.

Baker F. \& Bell C. (1999). Issues in the psychiatric treatment of African Americans. Psychiatric Service, 50(3), 362-368. 
Baronet, A. (1999). Factors associated with caregiver burden in mental illness: A critical review of the research literature. Clinical Psychological Review, 19(7), 819-841.

Beck, A., Ward, C., Mendelson, M., Mock, J., \& Erbaugh, J. (1961). An inventory for measuring depression. Archives of General Psychiatry 4, 561-571.

Bedard, M., Kolvuranta, A., \& Stuckey, A. (2004). Health impact on caregivers of providing informal care to a cognitively impaired older adult: Rural versus urban settings. Canadian Journal of Rural Medicine, 9(1), 15-23.

Beeson, R., Horton-Deutsch, S., Farran, C., \& Neundorfer, M. (2000). Loneliness and depression in caregivers of persons with Alzheimer's disease or related disorders. Issues in Mental Health Nursing, 21, 779-806.

Belasco, A., \& Sesso, R. (2002). Burden and quality of life of caregivers of hemodialysis patients. American Journal of Kidney Disease, 39(4), 805-812.

Berg, A., Palomako, H., Lonnqvist, J., Lehtihalmes, M., Phil, L., \& Kaste, M. (2005). Depression among caregivers of stroke survivors. Stroke, 36, 639-643.

Biegel, D., Sales, E., \& Schults, R. (1991). Theoretical perspectives on caregiving, Family Caregiving in Chronic Illness. Newbury Park: Sage Publications.

Blake, H., Lincoln, N., \& Clarke, D. (2003). Caregiver strain in spouses of stroke patients. Clinical Rehabilitation, 17, 312-317.

Bodenheimer, T., Lorig, K., Holman, H., \& Grumbach, K. (2002). Patient selfmanagement of chronic disease in primary care. Journal of American Medical Association, 288(19), 2496-2475.

Boerner, K., Horowitz, A., \& Schultz, R. (2004). Positive aspects of caregiving and adaptation to bereavement. Psychology and Aging, 19(4), 668-675.

Bookwala, J., \& Schultz, R. (2000). A comparison of primary stressors, secondary stressors, and depressive symptoms between elderly caregiving husbands and wives: The caregiver health effects study. Psychology and Again, 15(4), 607-616.

Braithwaite V. (2000). Contextual or general stress outcomes: Making choices through caregiving appraisals. Gerontologist, 40(6), 706-17.

Brazil, K., Bedard, M., Willison, K., \& Hode, M. (2003). Caregiving and its impact on families of the terminally ill. Aging and Mental Health, 7(5), 376-382. 
Brouwer, W., van Exel, N., van den Berg, B., van den Bos, G., \& Koopmanschap, M. (2005). Process utility from providing informal care: The benefits of caring. Health Policy, 74, 85-99.

Brouwer, W., van Exel, N., van den Berg, B., Dinant, H., Koopmanschap, M., \& van den Bos, G. (2004). Burden of caregiving: Evidence of objective burden, subjective burden, and quality of life impacts on informal caregivers of patients with rheumatoid arthritis. Arthritis and Rheumatism, 51(4), 570-577.

Brown, C., Schulberg, H., \& Madonia, M. (1996). Clinical presentations of major depression by African Americans and Whites in primary medical care practice. Journal of Affective Disorders, 41, 181-191.

Brown, C., Schulberg, H., Sacco, D., Perel, J., \& Houck, P. (1999). Effectiveness of treatments for major depression in primary care practice: A post doc analysis of outcomes for African American and White patients. Journal of Affective Disorders, 53, 185-192.

Bullock, K., Crawford, S., \& Tennstedt, S. (2003). Employment and caregiving: Exploration of African American caregivers. Social Work, 48(2), 150-162.

Burgener, S., \& Twigg, P. (2002). Relationships among caregiver factors and quality of life in care recipients with irreversible dementia. Alzheimer Disease and Associated Disorders, 16(2), 88-102.

Burns, N. \& Grove, S. (2001). Using statistics to predict, The Practice of Nursing Research: Conduct, Critique \& Utilization ( $4^{\text {th }}$ ed., pp. 547-561). Philadelphia, PA: W.B. Saunders.

Caap-Ahlgren, M., \& Dehlin, O. (2002). Factors of importance to the caregiver burden experienced by family caregivers of Parkinson's disease patients. Aging Clinical Experiemental Research, 14(5), 371-377.

Cain, C., \& Wicks, M. (2000). Caregiver attributes as correlates of burden in family caregivers coping with chronic obstructive pulmonary disease. Journal of Family Nursing, 6(1), 46-68.

Cain V., \& Kington R. (2003). Investigating the role of racial/ethnic bias in health outcomes. American Journal of Public Health. 93(2), 191-192.

Cannuscio, C., Jones, C., Kawachi, I., Coldidtz, G., Berkman, L., \& Rimm, E. (2002). Reverberations of family illness: A longitudinal assessment of informal caregiving and mental health status in the nurses' health study. American Journal of Public Health, 92(8), 1305-1311. 
Carey, P., Oberst, M., McCubbin, M., \& Hughes, S. (1991). Appraisal and caregiving burden in family members caring for patients receiving chemotherapy. Oncology Nursing Forum, 18, 1341-1348.

Chadiha, L., Adams, P., Biegel, D., Auslander, W., \& Gutierrez, L. (2004). Empowering African American women informal caregivers: A literature synthesis and practice strategies. Social Work, 49(1), 97-108.

Chico, A., Gauthier, A., Calvo, A., Ghiglione, P., \& Mutani, R. (2005). Caregiver burden and patients' perception of being a burden in ALS. Neurology, 64, 1780-1782.

Chiriboga, D., Weiler, P., \& Nielsen, J. (1998). The stress of caregivers. The Journal of Applied Social Sciences, 13(1), 119-141.

Choo, W., Low, W., Karina, R., Poip, P., Ebenezer, E., \& Prince, M. (2003). Social support and burden among caregivers of patients with dementia in Malaysia. Asia Pacific Journal of Public Health, 15(1), 23-29.

Clark, C., Mahoney, J., Clark, D., \& Eriksen, L. (2002). Screening for depression in hepatitis $\mathrm{C}$ population: The reliability and validity of the Center for Epidemiologic Studies Depression Scale (CES-D). Journal of Advanced Nursing, 40(3), 361-369.

Clark, D., \& Donovan, J. (1994). Reliability and validity of the Hamilton Anxiety Rating Scale in an adolescent sample. Journal of American Academy Children and Adolescent Psychiatry, 33(3), 354-360.

Clyburn, L., Stones, M., Hadjistavropoulos, T., \& Tuokko, H. (2000). Predicting caregiver burden and depression in Alzheimer's disease. Journal of Gerontology: Social Sciences, 55B(1), S2-S13.

Cochrane, J., Goering, P., \& Rogers, J. (1997). The mental health of informal caregivers in Ontario: An epidemiological survey. American Public Health Association, 87(12), 2002-2007.

Cohen, C., Colantonio, A., \& Vernich, L. (2002). Positive aspects of caregiving: Rounding out the caregiver experience. International Journal of Geriatric Psychiatry, 17, 184-188.

Cohen, J. (1988). Multiple regression and correlation analysis. Statistical power analysis for the behavioral science $\left(2^{\text {nd }}\right.$ ed., pp. 407-465). Hillsdale, New Jersery: Lawrence Erlbaum Associates.

Connell, C., \& Gibson, G. (1997). Racial, ethnic, and cultural differences in dementia caregiving: Review and analysis. The Gerontologist, 37, 355-364. 
Covinsky, K., Eng., G., Lui, S., Sands, L., Sehgal, A., Walter, L., Wieland, D., Eleazer, G., \& Yaffe, K. (2001). Reduced employment in caregivers of frail elders: Impact of ethnicity, patient clinical characteristics and caregiver characteristics. Journal of Abnormal Biological Science Medical Science, 56(11), M707-713.

Covinsky, K., Newcomer, R., Fox, P., Wood, J., Sands, L., Dane, K., \& Yaffe, K. (2003). Patient and caregiver characteristics associated with depression in caregivers of patients with dementia. Journal of General Internal Medicine, 18, 1006-1014.

Cox, C. (1999). Race and caregiving: Patterns of service used by African American and White caregivers of persons with Alzheimer's disease. Journal of Gerontology: Social Works, 32(2), 5-19.

Coyne, J., \& Smith, D. (1991). Couples coping with a myocardial infarction: A contextual perspective on wives' distress. Journal of Personality and Social Psychology and Aging, 61(3), 404-412.

Croog, S., Budilovsky, A., Burleson, J., \& Baume, R. (2001). Vulnerability of husband and wife caregivers of Alzheimer disease patients to caregiving stressors. Alzheimer Disease and Associated Disorders, 15(4), 201-210.

Croog, S., Burleson, J., Sudilovsky, A., \& Baume, R. (2006). Spouse caregivers of Alzheimer patients: Problem responses to caregiver burden. Aging Mental Health, 10(2), 87-100.

Crotty, P., \& Kulys, R. (1986). Are schizophrenics a burden to their families? Significant others' views. Health and Social Work, 3(11), 173-188.

Crum, R., \& Anthony, J. (2000). Educational level and risk for alcohol abuse and dependence: Differences by race and ethnicity. Ethnicity and Disease, 10(1), 39512.

Daeppen, J., Yersin, B., Landry, U., Pecound, A., \& Decrey, H. (2000). Reliability and validity of the Alcohol Use Disorders Identification Test (AUDIT) imbedded within a general health risk screening questionnaire: Results of a survey in 322 primary care patients. Alcohol Clinical Experiential Research, 24, 659-665.

Daniel, S. (2005). Chronic hepatitis C treatment patterns in African American patient: An update. American Journal of Gastroenterology, 100, 716-722.

Dawe, S., Seinen, A., \& Kavangh, D. (2000). An examination of the utility of the AUDIT in people with schizophrenia. Journal of Studies on Alcohol, 61(5), 744-750. 
Dawson, D. (2003). Methodological issues in measuring alcohol use. Alcohol Research and Health, 27(1) 18-29.

Devins G. \& Edwards P., (1988). Self-efficacy and smoking reduction in chronic obstructive pulmonary disease. Behavior Research and Therapy, 26(2):127-35.

Diehl, A. (2002). Liver disease in alcohol abusers: Clinical perspective. Alcohol, 27(1), 711.

Dilworth-Anderson, P., William I., \& Gibson, B. (2002). Issues of race, ethnicity and culture in caregiving research: A 20 year review (1980-2000). The Gerontologist, 42(2), 237-272.

Dilworth-Anderson, P., Williams, S., \& Cooper, T. (1999). The contexts of experiencing emotional distress among family caregivers to elderly African Americans. Family Relations, 48, 391-397.

Dilworth-Anderson, P., Williams, S. \& Cooper, T. (1999). Family caregiving to elderly African Americans: Caregiver types and structures. Journal of Gerontology: Social Sciences, 54B (4), S237-S241.

Dixit, A., \& Crum, R. (2000). Prospective study of depression and the risk of heavy alcohol use in women. The American Journal of Psychiatry, 157, 751-758.

Donelan, K., Falik, M., \& DesRoches, C. (2001). Caregiving: Challenges and implications for women's health. Women's Health Issues, 11(3), 185-200.

Donelan, K., Hill, C., Hoffman, C., Scoles, K., Feldman, P., Levine, C., \& Gould, D. (2002). Challenged to care: Informal caregivers in a changing health system. Health Tracking, 21(4), 222-231.

Dura, J., Stukenberg, K., \& Kiecolt-Glaser, Jr. (1991). Anxiety and depressive disorders in adult children caring for demented parents. Psychology and Aging, 6(3), 467473.

Dyck, D., Short, R., \& Vitaliano, P. (1999). Predictors of burden and infectious illness in schizophrenia caregivers. Psychosomatic Medicine, 61, 411-419.

Eaton, W., Neufeld, K., Chen, L., \& Cai, G. (2000). A comparison of self-report and clinical diagnostic interviews for depression: Diagnostic interview schedule and schedules for clinical assessment in neuropsychiatry in the Baltimore epidemiologic catchment area follow-up. Archives General Psychiatry, 57(3), 217-222. 
Edwards, P., Roberts, I., Clarke, M., DiGuiseppi, C., Pratap, S., Wentz, R., \& Kwan, I. (2002). Increasing response rates to postal questionnaires: Systematic review. British Medical Journal, 324(7347), 1168-1169.

Ekwall, A., Sivberg, B., \& Hallberg, I. (2004). Dimensions of informal care and quality of life among elderly family caregivers. Scandinavian Journal of Caring Science, 18 (239-248).

Faison, K., Faria, S., \& Frank, D. (1999). Caregivers of chronically ill elderly: Perceived burden. Journal of Community Health Nursing, 16(4), 243-253.

Fawcett, J. (1997). The detection and consequences of anxiety in clinical depression. Journal of Clinical Psychiatry, 58 (suppl 8), 35-40.

Fawcett, J. (2000). Analysis and evaluation of contemporary nursing knowledge: Nursing models and theories. Philadelphia, Pa: F.A. Davis.

Flaskerud, J., Carter, P., \& Lee, P. (2000). Distressing emotions in female caregivers of people with AIDS, age related dementias, and advanced-stage cancers. Perspectives in Psychiatric Care, 36(4), 131-130.

Forman, L., Lucey, M. (2001). Predicting the prognosis of chronic liver disease, An evolution from CHILD to MELD: Mayo End Stage Liver Disease. Hepatology, $33(2), 473-475$.

Fortis Report: National Family Caregiving Association. (1998). Family caregiving demands recognition: Fortis long term care and adult care. Retrieved August 21, 2003, from http://www.nfcacares.org./National/Family/Caregivers/Association .html.

Fredman, L., Daly, M., \& Lazur, A. (1995). Burden among White and Black caregivers to elderly adults. Journal of Gerontology: Social Sciences, 50B(2), 119-117.

Gallicchio, L., Siddiqi, N., Langenberg, P., \& Baumgarten, M. (2002). Gender differences in burden and depression among informal caregivers of demented elders in the community. International Journal of Geriatric Psychiatry, 17(2), 154-163.

Garand, L., Dew, M., Eazor, L., DeKosky, S., \& Reynolds, C. (2005). Caregiving burden and psychiatric morbidity in spouses of persons with mild cognitive impairment. International Journal of Geriatric Psychiatry, 20, 512-522.

Given, B., Wyatt, G., Given, C., Sherwood, P., gift, A., Devoss, D., \& Rahbar, M. (2004). Burden and depression among caregivers of patients with cancer at the end of life. Oncology Nursing Forum, 31(6), 1105-1116. 
Given, C., Given, B., Stommel, M., Collins, C., King, S., \& Franklin, S. (1992). The Caregiver Reaction Assessment (CRA) for caregivers to persons with chronic physical and mental impairments. Research in Nursing and Health, 15, 271-283.

Grunfeld, E., Coyle, D., Whelan, T., Clinch, J., Reyno, L., Earle, C., William, A., Viola, R., Coristine, M., Janz, T., \& Glossop, R. (2004). Family caregiver burden: Results of a longitudinal study of breast cancer patients and their principal caregivers. Canadian Medical Association Journal, 170(12), 1795-1801.

Guy, W. (1976). Assessment Manual for psychopharmacology. U.S. Department of Health and Human Services publication (Adm), pp 218-222, Rockville, MD.

Habib, A., Bond, W., \& Heuman, D. (2001). Long-term management of cirrhosis. Postgraduate medicine, 109(3), 1-13.

Haley, W., LaMonde, L., Han, B., Burton, A., \& Schonwetter, R. (2003). Predictors of depression and life satisfaction among spousal caregivers in hospice: Application of a stress process model. Journal of Palliative Medicine, 6(2), 215-224.

Haley, W., Roth, D., Coleton, M., Ford, G., \& West, C. (1996). Appraisal, coping, and social support as mediators of well-being in Black and White family caregivers in patients with Alzheimer's disease. Journal of Consulting Clinical Psychology, 64, 121-129.

Haley, W., West, C., Wadley, V., Ford, G., West, C., Collins, R., \& Sobe, T. (1995). Psychological, social, and health impact of caregiving: A comparison of Black and White dementia caregivers and non caregivers. Psychology and Again, 10(540-552).

Halpern, S., Ubel, P., Berlin, J., \& Asch, D. (2002). Randomized trial of 5 dollars versus 10 dollars monetary incentives, envelope size, and candy to increase physician response rates to mailed questionnaires. Medical Care, 40(9), 834-839.

Hamilton, M. (1959). The assessment of anxiety states by rating. British Journal of Medical Psychology, 32, 50-55.

Harris, T. T. (2003). Burden and health in caregivers of persons with kidney disease. Unpublished doctoral dissertation, University of Tennessee, Memphis.

Harris, T., Thomas, C., Wicks, M., Faulkner, M. \& Hathaway, D. (2000). Subjective burden in young and older African American caregivers of patients with end stage renal disease awaiting transplant. Nephrology Nursing Journal, 27(4), 383-391. 
Hathaway, S., \& McKinley, J. (1930). Minnesota Multi-phasic Personality Inventory (MMPI). Minneapolis, Minnesota: University of Minnesota Press.

Hecht, M., Graesel, E., Tigges, S., Hillemacher, T., Winterholler, M., Hilz, M., Heuss, D., \& Neundorfer, B. (2003). Burden of care in amyotrophic lateral sclerosis. Palliative Medicine, 17(327-333).

Hinrichsen, G., \& Ramirez, M. (1992). Black and White dementia caregivers: A comparison of their adaptation, adjustment and service utilization. Gerontologist, $32,35-381$.

Hinton, W., \& Levkoff, S. (1999). Constructing Alzheimer's: Narratives of lost identities, confusion and loneliness in old age. Culture, Medicine, and Psychiatry, 23, 453475.

Holahan, C., Moose, R., Holahan, C., Cronkite, R., \& Randall, P. (2003). Drinking to cope and alcohol use and abuse in unipolar depression: A 20 year model. Journal of Abnormal Psychology, 112(1), 159-165.

Hurcom, C., Copello, A., \& Orford, J. (2000). The family and alcohol: Effects of excessive drinking and conceptualizations of spouses over recent decades. Substance Use Misuse, 35(4), 473-502.

Institute of Medicine. (Ed.). (2002). Unequal Treatment: Confronting Racial \& Ethnic Disparities in Health. Washington: National Academic Press.

Jacobi, C., van den Berg, B., Boshuizen, H., Rupp, I., Dinant, H., \& van den Bos, G. (2003). Dimension-specific burden of caregiving among partners of rheumatoid arthritis patients. Rheumatology, 42, 12226-1233.

Janevic, M., \& Connell, C. (2001). Racial, ethnic, and cultural differences in the dementia caregiving experience: Recent findings. Gerontologist, 41(3), 334-347.

Judd, L. Paulus, M., Wells, K., \& Rapaport, M. (1996). Socioeconomic burden of subsyndromal depressive symptoms and major depression in a sample of the general population. American Journal of Psychiatry, 153(11), 1411-1417.

Kaneda, Y., \& Fuji, A. (2000). The relation between anxiety and depressive symptoms in normal subjects and patients with anxiety and/or mood disorders. Journal of Medical Investigations, 47, 14-18.

Kaplan, L., \& Boss, P. (1999). Depressive symptoms among spousal caregivers of institutionalized mates with Alzheimer's: Boundary ambiguity and mastery as predictors. Family Process, 38(1), 85-103. 
Karlawish, J., Casarett, D., Klocinski, J., \& Clark, C. (2001). The relationship between caregivers' global ratings of Alzheimer's disease patients' quality of life, disease severity, and the caregiving experience. Journal of American Geriatric Society, 49, 1066-1070.

Kerlinger, F., \& Lee, H. (2000). Foundations of behavioral research $\left(4^{\text {th }}\right.$ ed). Fort Worth, Texas: Harcourt College Publishers.

King, A., Bernardy, N., \& Hauner, K. (2003). Stressful events, personality, and mood disturbance: Gender differences in alcoholics and problem drinkers. Addictive Behavior, 28(1), 171-187.

Kneipp, S., Castleman, J., \& Gailor, N. (2004). Informal caregiving burden: An overlooked aspect of the lives and health of women transitioning from welfare to employment. Public Health Nursing, 2(1), 24-31.

Knight, B., Silverstein, M., McCallum, T., \& Fox, L. (2000). A psychological stress and coping model for mental health among African American caregivers in southern California. Journal of Gerontology: Psychological Sciences, 55B, 142-150.

Koff, R. (1988). Chronic hepatitis C: Early intervention. Hospital Practice, 33(6), 101$105,108,111-114$.

Kozachik, S., Given, C., Given, B., Pierce, S., Faouzi, A., Rawl, S., \& Champion, V. (2001). Improving depressive symptoms among caregivers of patients with cancer: Results of a randomized clinical trial. Oncology Nursing Forum, 28(7), 1149-1157.

Kruszon-Moran, D., \& McQuillan, G. (2005). Seroprevalence of six infectious diseases among adults in the United States by race/ethnicity: Data from the third National Health and Nutrition Examination Survey, 1988-1994.

Laidlaw, T., Coverdale, J., Falloon, I., \& Kydd, R. (2002). Caregivers' stresses when living together or apart from patients with chronic schizophrenia. Community Mental Health Journal, 38(4), 303-310.

Lawrence, R., Tennstedt, S., \& Assmann, S. (1998). Quality of the caregiver-care recipient relationship: Does it offset negative consequences of caregiving for family caregivers. Psychology and Aging. 13(1), 150-158.

Lawton, M., Rajagppal, D., Brody, E., \& Kleban, M. (1992). The dynamics of caregiving of a demented elder among Black and White families. Journal of Gerontology: Social Sciences, 47, S156-S164. 
Lim, J. \& Zebrack, B. (2004). Caring for family members with chronic physical illness: A critical review of caregiver literature. Health Quality Life Outcomes, 17(2), 50.

Lopez, J., Lopez-Arreta, J., \& Crespo, M. (2005). Factors associated with the positive impact of caring for elderly and dependent relatives. Archives Gerontology and Geriatrics, 41(1), 81-94.

Macera, C., Eakder, E., Goslar, P., Deandrade, S., Williamson, J., Cornman, C., \& Jannarone, J. (1992). Ethnic differences in the burden of caregiving. The American Journal of Alzheimer's Disease and related Disorders \& Research, 7(5) 4-7.

Mant, J., Carter, J., Wade, D., \& Winner, S. (2000). Family support for stroke: A randomized controlled trial. Lancet, 356, 808-813.

McCann, Hebert, L., Beckett, L., Morris, M., Scherr, P., \& Evans, D. (2000). Comparisons of informal caregiving by black and white older adults in a community population. Journal of American Geriatrics Society, 48(12), 1-10.

McCullagh, E., Brigstocke, G., Donaldson, N., \& Kalra, L. (2005). Determinants of caregiving burden and quality of life in caregivers of stroke patients. Stroke, 36, 2181-2186.

McNally, S., Ben-Shlomo, Y., \& Newman, S. (1999). The effects of respite care on informal carers' well-being: A systematic review. Disability and Rehabilitation, 21(1), 1-14.

Menon, N., Gores, G., \& Shah, V. (2001). Pathogenesis, diagnosis and treatment of alcoholic liver disease. Mayo Clinic Proceedings, 76(10), 1021-1029.

Miller, B., Campbell, R., Rarran, C., Kaurman, J., \& Davis, L. (1995). Race, control, mastery, and caregiver distress. Journal of Gerontology: Social Sciences, 50B, S374-S382.

Montgomery, R., Gonyea, J., \& Hooyman, N. (1985).Caregiving and the experience of subjective and objective burden. Family Relations, 34, 19-26.

Mort, J., Gasper, P., Juffer, D., \& Kovarna, M. (1996). Comparison of psychotropic agent use among rural elderly caregivers and non caregivers. The Annals of Pharmocotherapies, 30(6), 583-585.

Motenko, A. (1998). The frustration, gratifications and well-being of dementia caregivers. The Gerontologist, 29(2), 166-172. 
Moylan, C., \& Muir, A. (2005). Treatment of hepatitis C in special populations. Clinics in Liver Disease, 9(4), 567-577.

Nagatomo, I., Akasaki, Y., Uchida, M., Tominaga, M., Hashiguchi, W., \& Takegawa, M. (1999). Gender of demented patients and specific family relationship of caregivers to patients influence mental fatigue and burdens of relatives as caregivers. International Journal of Geriatric Psychiatry, 14, 618-625.

National Institute of Mental Health . (1998). Depression. Washington, DC: Author. Retrieved July 27, 2004, from http://www.nimh.nih.gov/healthinformation/ depressionmenu.cfm.

Navaie-Waliser, M., Spriggs, A., \& Feldman, P. (2002). Informal caregiving: Differential experiences by gender. Medical Care, 40(12), 1249-1259.

Nguyen, H., Tirolo-Kitner, M., Evans, M., \& Zonderman, A. (2004). Factorial invariance of the CES-D in low socioeconomic status African Americans compared with a nationally representative sample. Psychiatry Research, 126, 177-187.

Nickel, R., Wunsch, A., Egle, U., Lohse, A., \& Otto, G. (2002). The relevance of anxiety, depression, and coping in patients after transplantation. Liver Transplantation, $8(1), 63-71$.

Nijboer, C., Triemstra, M., Tempelaar, R., Sanderman, R., \& Van den Bos, G. (1999). Determinants of caregiving experiences and mental health of partners of cancer patients. Cancer, 86(4), 577-588.

Nutt, D. (1997). Management of patients with depression associated with anxiety symptoms. Journal of Clinical Psychiatry, 58 suppl 8, 11-16.

Patel, K., Muir, A., McHutchison, J. (2006). Diagnosis and treatment of chronic C infection. BMJ, 332, 1013-1017.

Pavalko, J., \& Artis, J. (1997). Women's caregiving and paid work: Casual relationships in late midlife. Journal of Gerontology: Series B Psychological Science and Social Sciences, 52(4), SS170-179.

Pearlin, L., Mullan, J., Semple, S., \& Skaff, M. (1990). Caregiving and the stress process: An overview of concepts and their measures. The Gerontologist, 30(5), 583-594.

Peden, A., Rayens, M., Hall, L., \& Grant, E. (2005). Testing an intervention to reduce negative thinking, depressive symptoms, and chronic stressors in low-income single mothers. Journal of Nursing Scholarship, 37(3), 268-274. 
Perlick, D. (2001). Special section on stigma as a barrier to recovery: Introduction. Psychiatric Services, 52(12), 1613-1632.

Picot, S. (1995). Rewards, costs and coping of African American caregivers. Nursing Research, 44(3), 147-152.

Picot, S., Debanne, S., Namazi, K., \& Wykle, M. (1997). Religiosity and perceived rewards of black and white caregivers. The Gerontologist, 37(1), 89-101.

Pinquart, M., \& Sorensen, S. (2004). Associations of caregiver stressors and uplifts with subjective well-being and depressive mood: A meta-analytic comparison. Aging and Mental Health, 8(5), 438-449.

Pinquart, M., \& Sorenson, S. (2006). Gender differences in caregiver stressors, social resources, and health: An updated meta-analysis. Journals of Gerontology Series B, Psychological Sciences and Social Sciences, 61(1), 33-45.

Pirraglia, P., Bishop, D., Herman, D., Trisvan, E., Lopez, R., Torgersen, C., Van Hof, A., Anderson, B., Miller, I., \& Stein, M. (2005). Caregiver burden and depression among informal caregivers of HIV-infected individuals. Journal of General Internal Medicine, 20, 510-514.

Polen, M., \& Green, C. (2001). Caregiving, alcohol use, and mental health symptoms among HMO members. Journal of Community Health Nursing, 26(4), 285-301.

Polivka, L. (2001). Paying family members to provide care: Policy considerations for states (Policy Brief No 7): Family Caregiver Alliance.

Preuss, U., Schuckit, M., Smith, T., Barnow, S., \& Danko, G. (2002). Mood and anxiety symptoms among 140 children from alcoholic and control families. Drug Alcohol Dependence, 67(3), 2335-242.

Pyrsopoulos, N., Jeffers, L. (2005). Chronic hepatitis C in African Americans. Clinics in Liver Disease, 9, 427-438.

Radloff, L. (1977). The CES-D: A self-report depression scale for research in general population. Applied Psychological Measurement, 1(3), 385-401.

Raskins, A., Schulterbrandt, J., Reatig, N., \& McKeon, J. (1969). Replication of factors of psychopathology in interview, ward behavior, and self report ratings of hospitalized depressives. Journal of Nervous and Mental Disease, 148, 87-96.

Reardon, S., \& Buka, S. (2002). Differences in onset and persistence of substance abuse and dependence among whites, blacks, and Hispanics. Public Health Report, 117 (Suppl 1), S51-59. 
Rees, J., O'Boyle, C., \& MacDonagh R. (2001). Quality of life: impact of chronic illness on the partner. Journal of Royal Society of Medicine, 94(11), 563-566.

Reid, C., Fiellin, D., \& O’Connor, P. (1999). Hazardous and harmful alcohol consumption in primary care. Archives of Internal Medicine, 159(15), 1681-1689.

Reinhart, D., \& Allen, J. (2002). The alcohol use disorder identification test (AUDIT): A review of recent research. Alcoholism Clinical and Experimental Research, 26(2), 272-279.

Riedel, S., Fredman, L., \& Langenberg, P. (1998). Associations among caregiver difficulties, burden and rewards in caregivers to older post-rehabilitation patient. Journal of Gerontology: Psychological Sciences, 53B(3), 165-174.

Rivera-Navarro, J., Morales-Gonazlez, J., Benito-Leon, J., \& GEDMA, M.D.D.G. (2003). Informal caregiving in multiple sclerosis patients: Data from the Madrid demyelinating disease study group. Disability and Rehabilitation, 25(18), 10571064.

Roberts, J. (2000). Who pays the bills and who makes the profit in treating chronic illness? Western Journal of Medicine, 172, 76-77.

Robinson, K., Adkisson, P., \& Weinrich, S. (2001). Problem behavior, caregiver reactions, and impact among caregivers of persons with Alzheimer's disease. Journal Advanced Nursing, 36(4), 573-582.

Roff, L., Burgio, L., Gitlin, L., Nichols, L., Chaplin, W., \& Hardin, J.M. (2004). Positive aspects of Alzheimer's caregiving: The role of race. Journal of Gerontology: Psychological Sciences, 59B (4), 573-582.

Ross, R., Fortney, J., Lancaster, B., \& Booth, B. (1998). Focus on women: Age, ethnicity and comorbidity in a national sample of hospitalized alcohol-dependent women veterans. Psychiatric Services, 49, 663-668.

Roth, L., Haley, W., Owen, J., Clay, O., \& Goode, K. (2001). Latent growth models of the longitudinal effects of dementia caregiving: A comparison of African American and White family caregivers. Psychology and Aging, 16(3), 427-436.

Sarna, L., \& Chang, B. (2000). Colon cancer screening among older women caregivers. Cancer Nursing, 23(2), 109-116.

Saunders, J., Aasland, O., Barbor, T., de la Fuente, J., Grant, M., \& McCubbin, M. (1993). Development of the Alcohol Use Disorders Identification Test (AUDIT): 
WHO collaborative project on early detection of person with harmful alcohol consumption. II. Addictions, 88, 791-804.

Savage, S., \& Bailey, S. (2004). The impact of caring on caregivers' mental health: A review of the literature. Australian Health Review, 27(11), 111-115.

Scherbring, M.(2002). Effect of caregiver perception of preparedness on burden in an oncology population. Oncology Nursing Forum, 29(6), 1-14.

Schrag, A., Hovris, A., Morley, D., Quinn, N., \& Jahanshahi, M. (2005).Caregiverburden in Parkinson's disease is closely associated with psychiatric symptoms, falls, and disability. Parkinsonism \& Related Disorders, 20(40), 1-7.

Schroeder, M. (1990). Diagnosing and dealing with multicollinearity. Western Journal of Nursing Research, 12(2), 175-187.

Schutte, K., Moos, R., \& Brennan, P. (1995). Depression and drinking behavior among women and men: A three-wave longitudinal study of older adults. Journal of Consulting Clinical Psychology, 63(5), 810-822.

Schwarz K., \& Roberts B. (2000). Social support and strain of family caregivers of older adults. Holistic Nursing Practice, 14(2), 77-90.

Seltzer, M., \& Wailing, L. (1996). The transitions of caregiving: Subjective and objective definitions. Gerontologist, 36(5), 614-626.

Sharp L., \& Lipsky, M. (2002). Screening for depression across the lifespan: A review of measures for use in primary care settings. American Family Physician, 66(6), $952-955$.

Shifren, K. (2001). Early caregiving and adult depression. Good news for young caregivers. The Gerontologist, 41(2), 188-190.

Singh, G., \& Hoyert, D. (2000). Social epidemiology of chronic liver disease and mortality in the United States, 1935-1977: Trends and differentials by ethnicity, socioeconomic status, and alcohol consumption. Human Biology, 72(5), 801-820.

Sisk, R.(2000). Caregiving burden and health promotion. International Journal of Nursing, 37, 37-43.

Sitzia, J., \& Wood, N. (1998). Response rate in patient satisfaction research: An analysis of 210 published studies. International Journal for Quality in Health Care, 10(4), 311-317. 
Sjoberg, L. (1998). Risk perception of alcohol consumption. Alcoholism Clinical and Experimental Research, 22(7 suppls), 277S-284S.

Sochalski, J., \& Patrician, P. (1998). An overview of health care spending patterns in the United States: Using national data sources to explore trends in nursing services. Online Journal of Issues in Nursing, Retrieved August 21, 2004 http://www.nursingworld.org/ojin/tpc6.

Son, G.-R., Zauszniewski, J., Wykle, M., \& Picot, S. (2000). Translation and validation of caregiving satisfaction scale into Korean. Western Journal of Nursing Research, 22(5), 609-622.

Spurlock, W. (2005). Spiritual well-being and caregiver burden in Alzheimer's caregivers. Geriatric Nursing, 26(3), 154-161.

Sterritt, P.,\& Pokorny M. (1998). African-American caregiving for a relative with Alzheimer's disease. Geriatric Nursing, 19(3), 133-134.

Stolz, P., Uden, G., \& Willman, A. (2004). Support for family careers who care for an elderly person at home: A systematic literature review. Scandinavian Journal of Caring Science, 18, 111-119.

Substance Abuse Mental Health Services Administration. (2004). National Household Survey on Drug Abuse. Retrieved June, 15, 2006, from http://www.oas.samhsa .gov/nhsda.htm.

Tam, T., Weisner, C., \& Mertens, J. (2000). Demographic characteristics, life context, and patterns of substance use among alcohol-dependent treatment clients in a health maintenance organization. Alcoholism Clinical and Experimental Research, 24(2), 1803-1810.

Teel, C., Duncan, P., \& Lai, S. (2001). Caregiving experiences after a stroke. Nursing Research, 50(1), 53-60.

Thompson, E., \& Doll, W. (1982). The burden of families coping with the mentally ill: An invisible crisis. Family Relations, 31, 379-388.

Tsai, P., \& Jirovec, M. (2005). The relationship between depression and other outcomes of chronic illness caregiving. BMC Nursing, 4(3), 1-10.

Tucker, J., \& Vuchinich, R. (2000). Creating a research context for reducing risk and obtaining informed consent in human alcohol studies. Psychology of Addictive Behavior, 14(4), 319-324. 
Ude-Alvarez, F., Valdes, C., Estenbanez, C., \& Rebollo, P. (2004). Health-related quality of life of family caregivers of dialysis patients. Journal of Nephrology, 17, 841-850.

United States Census Bureau (2000). State \& County QuickFacts. Retrieved October 15, 2006, from http://quickfacts.census.gov/qfd/states/47/4748000.html.

Van Oers, J., Bongers, I., Van De Goor, L, \& Garretsen, J. (1999). Alcohol consumption, alcohol-related problems, problem drinking, and socioeconomic status. Alcohol and Alcoholism, 14(1), 78-88.

Vanderwerker, L., Laff, R., Kadan-Lottick, N., McColl, S., \& Prigerson, H. (2005). Psychiatric disorders and mental health service use among caregivers of advanced cancer patients. Journal of Clinical Oncology, 23(28), 6899-6907.

Vedhara, K., Shanks, N., Anderson, S., \& Lightman, S. (2000). The role of stressors and psychosocial variables in the stress process: A study of chronic caregiver stress. Psychosomatic Medicine, 62, 374-385.

Vitaliano, P., Russo, J., Young, H., Becker, J., \& Maiuro, R. (1991). The screen for caregiver burden. Gerontologist, 31(1), 76-83.

Wallsten, S. (2000). Effects of caregiving, gender, and race on the health mutuality and social supports of older adults. Journal of Aging Health, 12(1), 90-111.

Weitzner, M., Haley, W., Chen H. (2000). The family caregiver of the older cancer patient. Hematology/Oncology Clinic of North America, 14(1), 269-81.

Wells, K., Klap, R., Kioke, A., \& Sherbourne, C. (2001). Ethnic disparities in unmet need for alcoholism, drug abuse, and mental health. American Journal of Psychiatry, $158,2027-2032$.

White, T., Townsend, A., \& Stephens, M. (2000). Comparisons of African American and White women in the parent care role. The Gerontologist, 40(6), 718-728.

Wicks, M., Milstead, J., Hathaway, D., \& Cetingok, M. (1998). Family caregivers' burden, quality of life, and health following patients' renal transplantation. Journal of Transplant Coordination, 8(3), 170-176.

Williams, A., Forbes, D., Mitchell, D., Mitchell, J., Essar, M., \& Corbett, B. (2003). The influence of income on the experience of informal caregiving: Policy implications. Health Care for Women International, 24, 280-291.

Williams, D. (1996). Race/ethnicity and socioeconomic status: Measurement and methodology issues. International Journal of Health Services, 26(3), 483-505. 
Williams, D., \& Williams-Morris, R. (2000). Racism and mental health: The African American experience. Ethnicity and Health, 5(3/4), 243-268.

Wood, J., \& Parham, I. (1990). Coping with perceived burden: Ethnic and cultural issues in Alzheimer's family caregiving. Journal of Applied Gerontology, 9, 325-339.

Yee, J., \& Schultz, R. (2000). Gender differences in psychiatric morbidity among family caregivers: A review and analysis. The Gerontologist, 40, 147-164.

Yin, T., Zhou, Q., \& Bashford, C. (2002). Burden on family members: Caring for frail elderly: A meta-analysis on interventions. Nursing Research, 51(3), 199-208.

Young, R., \& Kahana, E. (1995). The context of caregiving and well being outcomes among African and Caucasian Americans. The Gerontologist, 25, 225-232.

Zarit S., Reever K., \& Bach-Peterson J. (1980) Relatives of the impaired elderly: correlates of feelings of burden. The Gerontologist, 20(6), 649-55.

Zarit, S., Todd, P., \& Zarit, J. (1985). Subjective burden of husbands and wives caregivers: A longitudinal study. The Gerontologist, 26(3), 260-266.

Zimmerman, M. (2003).Generalized anxiety disorder in patients with major depression: Is the DSM-IV's hierarchy correct? American Journal of Psychiatry, 160(3), 504515.

Zung, W. (1965). A self-rating depression scale. Archives of General Psychiatry, 12, 6370. 
APPENDICES 
Appendix A

Child Pugh Score 


\section{Child Pugh Score}

\begin{tabular}{llll}
\hline Total Serum Bilirubin & Points & Ascites & Points \\
\hline Bilirubin $<2 \mathrm{mg} / \mathrm{dl}$ & 1 point & No ascites & 1 point \\
Bilirubin 2 to $3 \mathrm{mg} / \mathrm{dl}$ & 2 points & Ascites controlled medically & 2 points \\
Bilirubin $3 \mathrm{mg} / \mathrm{dl}$ & 3 points & Ascites poorly controlled & 3 points \\
Serum Albumin & Points & Encephalopathy & Points \\
Albumin $<3.5 \mathrm{~g} / \mathrm{dl}$ & 1 point & No encephalopathy & 1 point \\
Albumin 2.8 to $3.5 \mathrm{~g} / \mathrm{dl}$ & 2 points & Encephalopathy controlled medically & 2 points \\
Albumin $>2.8 \mathrm{~g} / \mathrm{dl}$ & 3 points & Encephalopathy poorly controlled & 3 points \\
INR (ionized normalized ratio) & Points & & \\
INR $<1.70$ & 1 point & & \\
INR 1.70 to 2.20 & 2 points & & \\
INR $>2.20$ & 3 points & &
\end{tabular}

\section{Interpretation}

\section{Child Class A: 5 to 6 points}

1. Life expectancy, 15 to 20 years

2. Abdominal surgery peri-operative mortality, $10 \%$

\section{Child Class B: 7 to 9 points}

1. Indicated for liver transplantation evaluation

2. Abdominal surgery peri-operative mortality, $30 \%$

\section{Child Class C: 10 to 15 points}

1. Life expectancy, 1 to 3 years.

2. Abdominal surgery peri-operative mortality, $82 \%$. 
Appendix B

Family Caregiver Demographic Data Form 


\section{Family Caregiver Demographic Data Form}

Please read each question carefully. Some questions ask about you and some questions ask for information about the patient (your family member who has liver disease). You have to fill in the blank for some of the questions.

For other questions you have to circle your answer. Please answer every question.

Your Initials

Patient's Initials

Your Marital Status: (circle one answer)

Single $\quad$ Married Divorced Widowed

Your Gender: Male Female Patient's Gender: Male Female

Your Race Patient's Race

Your Employment Status: (Circle one answer)

Full-time Part-time Unemployed Retired Homemaker Student

Patient's Employment Status: (Circle one answer)

Full-time Part-time Unemployed Retired Homemaker Student

Your Occupation Your Years of Education

Your annual income (include total income of family if shared)

$\$$ per year

Has your income decreased since caring for the patient?

Circle one answer.

Yes No

What is your relationship to patient? I am the patient's (Circle one answer)

Spouse Child Parent Sibling Other 
Do you live with the patient? (Circle one answer) No No

Who assists the patient with activities of daily living on most days?

Activities of daily living include things like bathing and dressing.

(Circle one answer)

1. No assistance needed

2. I assist the patient

3. Other family or friend living in the household assist the patient

4. Other family or friend living outside the household assists the patient

5. A health care professional or health care worker assists the patient

How many years have you been providing care to the patient?

How long have you been married to the patient? years

How satisfied are you with the support that you get from family and friends? Circle one answer.

-Very Satisfied • $\bullet$ Satisfied $\bullet$ Somewhat Satisfied $\bullet$ Dissatisfied $\bullet$ Very Dissatisfied

Are you taking care of anyone in your family other than the person with liver disease? Circle one answer.

Yes No

Are you worried about your children?

Yes No

Have you ever been depressed? Circle one answer. $\quad$ No Yes

Has anyone in your family ever been depressed? No N 
Family Caregiver Demographic Data Form

Page 3

\begin{tabular}{|c|c|c|c|}
\hline \multicolumn{4}{|c|}{ Check if you have taken any of the following drugs } \\
\hline None & $\square$ & PCP & $\square$ \\
\hline Marijuana & $\square$ & LSD/hallucinogens & $\square$ \\
\hline Cocaine/crack & $\square$ & Barbiturates/sedatives & $\square$ \\
\hline Amphetamines/stimulants & $\square$ & Other street drugs & $\square$ \\
\hline Heroin & $\square$ & & \\
\hline \multicolumn{4}{|c|}{ If checked one or more of these drugs under what circumstances did you take it (them)? } \\
\hline \multicolumn{4}{|c|}{ When did you most heavily use drugs? } \\
\hline When was the last time yo & & & \\
\hline
\end{tabular}

\begin{tabular}{|c|c|c|c|}
\hline \multicolumn{4}{|c|}{ Check if you have had or have any of the following diseases } \\
\hline None & $\square$ & Respiratory problems & $\square$ \\
\hline Diabetes & $\square$ & Arthritis & $\square$ \\
\hline Hypertension & $\square$ & Cancer & $\square$ \\
\hline Heart disease & $\square$ & Ulcers, heartburn, constipation, diarrhea & $\square$ \\
\hline Hepatitis & $\square$ & Other medical problems & $\square$ \\
\hline \multicolumn{4}{|c|}{ Explain medical problems } \\
\hline \multicolumn{4}{|c|}{ Have you had any surgeries, if so when? } \\
\hline
\end{tabular}

\section{You Have Completed this Questionnaire \\ Patient's CPS \\ Patient's MELD}




\section{Appendix C}

Center for Epidemiological Studies Depression Scale 


\section{Center for Epidemiological Studies Depression Scale}

Instructions for questions: Below is a list of some of the ways you may have felt or behaved. Please indicate how often you have felt this way during the past week.

During the past week:

$\begin{array}{cl}\text { Rarely or } & \begin{array}{l}\text { Some of a } \\ \text { none of }\end{array} \\ \text { Little of the } \\ \text { (Less than 1 day) } & \text { Time } \\ \text { (1-2 days })\end{array}$
Occasionally or a Moderate Amount of the
Time (3-4 days)
Most or All of the Time (5-7 days)

1. I was bothered by things 0

that usually don't bother me.

2. I did not feel like eating; $\quad 0$

my appetite was poor.

3. I felt that I could not shake 0

off the blues even with help

from my family or friends.

4. I felt that I was just as

0

2

good as other people.

5. I had trouble keeping my

0

2

mind on what I was doing.

6. I felt depressed.

0

2

7. I felt that everything

0

I did was an effort.

8. I felt hopeful

about the future.

0

2

9. I thought my life had

0

2

been a failure.

10. I felt fearful.

0

2

11. My sleep was restless. 0

1

2

12. I was happy.

0

1

2 
Instructions for questions: Below is a list of some of the ways you may have felt or behaved. Please indicate how often you have felt this way during the past week.

\begin{tabular}{|c|c|c|c|c|}
\hline During the past week: & $\begin{array}{c}\text { Rarely or } \\
\text { none of } \\
\text { the time } \\
\text { (Less than } 1 \text { day) }\end{array}$ & $\begin{array}{l}\text { Some of a } \\
\text { Little of the } \\
\text { Time } \\
\text { (1-2 days) }\end{array}$ & $\begin{array}{l}\text { Occasionally } \\
\text { or a Moderate } \\
\text { Amount of the } \\
\text { Time (3-4 days) }\end{array}$ & $\begin{array}{l}\text { Most or All } \\
\text { of the } \\
\text { Time } \\
\text { (5-7 days) }\end{array}$ \\
\hline
\end{tabular}

13. I talked less than usual.

$0 \quad 1$

2

3

14. I felt lonely.

0

1

2

3

15. People were unfriendly.

0

1

2

3

16. I enjoyed life.

0

1

2

3

17. I had crying spells.

0

1

2

3

18. I felt sad.

0

1

2

3

19. I felt that people

0

1

2

disliked me.

20. I could not

0

1

2

Reverse Score 4, 8, 12, 16

Source. Radloff, L. (1977). The CES-D: A self-report depression scale for research in general population. Applied Psychological Measurement, 1(3), 385-401. @ Public Domain 


\section{Appendix D}

Hamilton Anxiety Rating Scale (HAM-A) 


\section{Hamilton Anxiety Rating Scale (HAM-A)}

The Hamilton Anxiety is a rating scale developed to quantify the severity of anxiety symptoms. Each item is rated on a 5-point scale, ranging from 0 (not present) to 4 (disabling). Please circle the response that applies to the symptoms you feel.

Symptoms Classification of Symptoms: 0-absent; 1-mild; 2-moderate; 3-severe; 4-Disabling

\begin{tabular}{|c|c|c|c|c|c|c|}
\hline & Symptoms & $\begin{array}{l}\text { Not } \\
\text { present }\end{array}$ & Mild & Moderate & Severe & Disabling \\
\hline 1. & $\begin{array}{l}\text { Anxious Mood } \\
\quad * \text { worries } \\
\quad * \text { anticipates worst }\end{array}$ & 0 & 1 & 2 & 3 & 4 \\
\hline 2. & 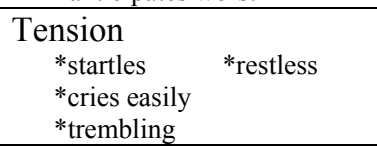 & 0 & 1 & 2 & 3 & 4 \\
\hline 3. & 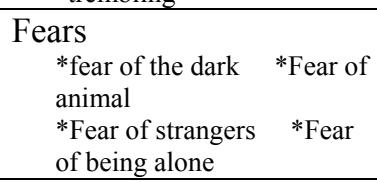 & 0 & 1 & 2 & 3 & 4 \\
\hline 4. & $\begin{array}{l}\text { Insomnia } \\
\text { *difficulty falling asleep or } \\
\text { staying asleep } \\
\text { *difficulty with nightmares }\end{array}$ & 0 & 1 & 2 & 3 & 4 \\
\hline 5. & $\begin{array}{l}\text { Intellectual } \\
\quad \text { *poor concentration } \\
\text { *memory impairment }\end{array}$ & 0 & 1 & 2 & 3 & 4 \\
\hline 6. & $\begin{array}{l}\text { Depressed Mood } \\
\text { *decreased interest in } \\
\text { activities } \\
\text { *anhedonia } \\
\text { *insomnia }\end{array}$ & 0 & 1 & 2 & 3 & 4 \\
\hline 7. & $\begin{array}{l}\text { Somatic Complaints- } \\
\text { muscular } \\
\quad \text { *muscle aches or pains } \\
\quad \text { *bruxism } \\
\end{array}$ & 0 & 1 & 2 & 3 & 4 \\
\hline 8. & $\begin{array}{l}\text { Somatic Complaints- } \\
\text { Sensory } \\
\quad \text { *tinnitus } \\
\quad \text { *blurred vision }\end{array}$ & 0 & 1 & 2 & 3 & 4 \\
\hline 9. & $\begin{array}{l}\text { Cardiovascular } \\
\text { Symptoms } \\
\text { *tachycardia } \quad * \text { chest } \\
\text { pain } \\
\quad \text { *palpitations } \\
\quad{ }_{\text {s }} \text { sensory of feeling faint }\end{array}$ & 0 & 1 & 2 & 3 & 4 \\
\hline
\end{tabular}


The Hamilton Anxiety is a rating scale developed to quantify the severity of anxiety symptoms. Eachitem is rated on a 5-point scale, ranging from 0 (not present) to 4 (disabling). Please circle the response that applies to the symptoms you feel.

Symptoms Classification of Symptoms: 0-absent; 1-mild; 2-moderate; 3-severe; 4-Disabling

\begin{tabular}{|c|l|c|c|c|c|c|}
\hline Symptoms & $\begin{array}{l}\text { Not } \\
\text { present }\end{array}$ & Mild & Moderate & Severe & Disabling \\
\hline 10. & $\begin{array}{l}\text { Respiratory Symptoms } \\
\text { *chest pressure *choking sensation } \\
\text { *Shortness of breath }\end{array}$ & 0 & 1 & 2 & 3 & 4 \\
\hline 11. & $\begin{array}{l}\text { Gastrointestinal Symptoms } \\
\text { *dysphagia } \\
\text { *nausea \& vomiting } \\
\text { *constipation } \\
\text { *weight loss }\end{array}$ & 0 & 1 & 2 & 3 & 4 \\
\hline 12. & $\begin{array}{l}\text { Genitourinary Symptoms } \\
\text { *urinary frequency or urgency } \\
\text { *dysmenorrheal } \\
\text { *impotence }\end{array}$ & 0 & 1 & 2 & 3 & 4 \\
\hline 13. & $\begin{array}{l}\text { Autonomic Symptoms } \\
\text { *dry mouth *pallor } \\
* \text { sweating }\end{array}$ & 0 & 1 & 2 & 3 & 4 \\
\hline 14. & $\begin{array}{l}\text { Behavior At Interview } \\
\text { *fidgets *paces *tremor }\end{array}$ & 0 & 1 & 2 & 3 & 4 \\
\hline
\end{tabular}

Source: Hamilton, M. (1959). The assessment of anxiety states by rating. British Journal of Medical Psychology, 32, 50-55. @ Public Domain 
Appendix E

Alcohol Use Disorders Identification Test 


\section{Alcohol Use Disorders Identification Test: Self-Report Version}

Patient: Because alcohol use can affect your health and care interferes with certain medications and treatments, it is important that we ask some questions about your use of alcohol. Your answers will remain confidential so please be honest. Place an $\mathrm{X}$ in one box that best describes your answer to each question.

\begin{tabular}{|c|c|c|c|c|c|}
\hline Questions & 0 & 1 & 2 & 3 & 4 \\
\hline $\begin{array}{l}\text { How often do you } \\
\text { have } \\
\text { a drink containing } \\
\text { alcohol? }\end{array}$ & Never & $\begin{array}{l}\text { Monthly or } \\
\text { less }\end{array}$ & $\begin{array}{l}\text { 2-4 times a } \\
\text { month }\end{array}$ & $\begin{array}{l}2-3 \text { times a } \\
\text { week }\end{array}$ & $\begin{array}{l}4 \text { or more } \\
\text { times a } \\
\text { week }\end{array}$ \\
\hline $\begin{array}{l}\text { How many drinks } \\
\text { containing } \\
\text { alcohol do you have } \\
\text { on a typical day } \\
\text { when you are } \\
\text { drinking? }\end{array}$ & 1 or 2 & 3 or 4 & 5 or 6 & 7 to 9 & 10 or more \\
\hline $\begin{array}{l}\text { How often do you } \\
\text { have six or } \\
\text { more drinks on one } \\
\text { occasion? }\end{array}$ & Never & $\begin{array}{l}\text { Less than } \\
\text { Monthly }\end{array}$ & Monthly & Weekly & $\begin{array}{l}\text { Daily or } \\
\text { almost daily }\end{array}$ \\
\hline $\begin{array}{l}\text { How often during } \\
\text { the last year have } \\
\text { you found that you } \\
\text { were not able to } \\
\text { stop drinking daily? }\end{array}$ & Never & $\begin{array}{l}\text { Less than } \\
\text { Monthly }\end{array}$ & Monthly & Weekly & $\begin{array}{l}\text { Daily or } \\
\text { almost daily }\end{array}$ \\
\hline $\begin{array}{l}\text { How often during } \\
\text { the last year have } \\
\text { you failed to do } \\
\text { what was normally } \\
\text { expected of daily } \\
\text { you because of } \\
\text { drinking? }\end{array}$ & Never & $\begin{array}{l}\text { Less than } \\
\text { Monthly }\end{array}$ & Monthly & Weekly & $\begin{array}{l}\text { Daily or } \\
\text { almost daily }\end{array}$ \\
\hline $\begin{array}{l}\text { How often during } \\
\text { the last year have } \\
\text { you needed a first } \\
\text { drink in the } \\
\text { morning to get } \\
\text { yourself daily } \\
\text { once you had } \\
\text { started? }\end{array}$ & Never & $\begin{array}{l}\text { Less than } \\
\text { Monthly }\end{array}$ & Monthly & Weekly & $\begin{array}{l}\text { Daily or } \\
\text { almost daily }\end{array}$ \\
\hline
\end{tabular}


Patient: Because alcohol use can affect your health and care interferes with certain medications and treatments, it is important that we ask some questions about your use of alcohol. Your answers will remain confidential so please be honest. Place an $\mathrm{X}$ in one box that best describes your answer to each question.

$\begin{array}{llllll}\begin{array}{l}\text { How often during the last } \\ \text { year have you had a feeling } \\ \text { of guilt or remorse after } \\ \text { drinking? }\end{array} & \text { Never } & \begin{array}{l}\text { Less than } \\ \text { Monthly }\end{array} & \text { Monthly } & \text { Weekly } & \begin{array}{l}\text { Daily or } \\ \text { almost } \\ \text { daily }\end{array} \\ \text { Questions } & 1 & 2 & 3 & 4 & 5\end{array}$

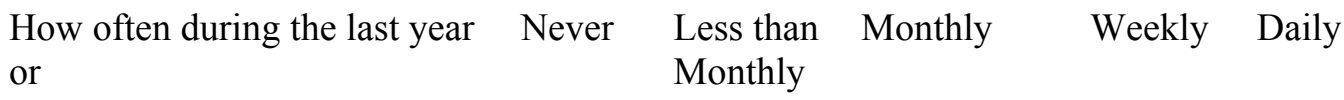

have you been unable to remember what happened the night before because of your drinking?

Have you or someone else No

Yes, but not

Yes been injured because of your drinking? in the last during year the last

Has a relative, friend, doctor, No Yes, but not year or other health care worker been concerned about your in the last Yes year during the last drinking or suggested you cut down? year

Total

Source. World Health Organization. WHO Press, Geneva Switzerland.@ Public Domain 


\section{Appendix F}

\section{Burden Interview}




\section{Burden Interview}

INSTRUCTIONS: The following is a list of statements, which reflect how people sometimes feel when taking care of another person. After each statement, indicate how often you feel that way, never, rarely sometimes, quite frequently, or nearly always. There are no rights or wrong answers.

1. Do you feel that your relative asks for more help than he or she needs?

0. Never 1. Rarely 2. Sometimes 3. Quite Frequently 4. Nearly Always

2. Do you feel that because of the time you spend with your relative that you don't have enough time for yourself?

0. Never 1. Rarely 2. Sometimes 3. Quite Frequently 4. Nearly Always

3. Do you feel stressed between caring for your relative and trying to meet other responsibilities for you family or work?

0. Never 1. Rarely 2. Sometimes 3. Quite Frequently 4. Nearly Always

4. Do you feel embarrassed over your relative's behavior?

0. Never 1. Rarely 2. Sometimes 3. Quite Frequently 4. Nearly Always

5. Do you feel angry when you are around your relative?

0. Never 1. Rarely 2. Sometimes 3. Quite Frequently 4. Nearly Always

6. Do you feel that your relative currently affects your relationship with other family members or friends in a negative way?

0. Never 1. Rarely 2. Sometimes 3. Quite Frequently 4. Nearly Always

7. Are you afraid what the future holds for your relative?

0. Never 1. Rarely 2. Sometimes 3. Quite Frequently 4. Nearly Always

8. Do you feel your relative is dependent upon you?

0. Never 1. Rarely 2. Sometimes 3. Quite Frequently 4. Nearly Always 
INSTRUCTIONS: The following is a list of statements, which reflect how people sometimes feel when taking care of another person. After each statement, indicate how often you feel that way, never, rarely sometimes, quite frequently, or nearly always. There are no rights or wrong answers.

9. Do you feel strained when you are around your relative?

0. Never 1. Rarely 2. Sometimes 3. Quite Frequently 4. Nearly Always

10. Do you feel your health has suffered because of your involvement with your relative?

0. Never 1. Rarely 2. Sometimes 3. Quite Frequently 4. Nearly Always

11. Do you feel that you don't have as much privacy as you would like, because of your relative?

0. Never 1. Rarely 2. Sometimes 3. Quite Frequently 4. Nearly Always

12. Do you feel that your social life has suffered because you are caring for your relative?

0. Never 1. Rarely 2. Sometimes 3. Quite Frequently 4. Nearly Always

13. Do you feel uncomfortable about having friends over, because of your relative?

0. Never 1. Rarely 2. Sometimes 3. Quite Frequently 4. Nearly Always

14. Do you feel that your relative seems to expect you to take care of him or her, as if you were the only one he or she could depend on?

0. Never 1. Rarely 2. Sometimes 3. Quite Frequently 4. Nearly Always

15. Do you feel that you don't have enough money to care for your relative, in addition to the rest of your expenses?

0. Never 1. Rarely 2. Sometimes 3. Quite Frequently 4. Nearly Always

16. Do you feel that you will be unable to take care of your relative much longer?

0. Never 1. Rarely 2. Sometimes 3. Quite Frequently 4. Nearly Always 
INSTRUCTIONS: The following is a list of statements, which reflect how people sometimes feel when taking care of another person. After each statement, indicate how often you feel that way, never, rarely sometimes, quite frequently, or nearly always. There are no rights or wrong answers.

17. Do you feel you have lost control of your life since your relative's illness?

0. Never 1. Rarely 2. Sometimes 3. Quite Frequently 4. Nearly Always

18. Do you wish you just leave the care of your relative to someone else?

0. Never 1. Rarely 2. Sometimes 3. Quite Frequently 4. Nearly Always

19. Do you feel uncertain about what to do about your relative?

0. Never 1. Rarely 2. Sometimes 3. Quite Frequently 4. Nearly Always

20. Do you feel you should be doing more for your relative?

0. Never 1. Rarely 2. Sometimes 3. Quite Frequently 4. Nearly Always

21. Do you feel you could do a better job in caring for your relative?

0. Never 1. Rarely 2. Sometimes 3. Quite Frequently 4. Nearly Always

22. Overall, how burdened do you feel in caring for your relative?

0. Not at all 1. A little 2. Moderately 3. Quite a bit 4. Extremely

Source. Zarit S., Reever K., \& Bach-Peterson J. (1980) Relatives of the impaired elderly: correlates of feelings of burden. The Gerontologist, 20(6), 649-55. Permission granted by Steven Zarit. 
Appendix G

Picot Caregiver Rewards Scale 


\section{Picot Caregiver Rewards Scale}

Now I'd like to talk to you about some of the ways people feel about caring for another person. Please tell me how you feel now about caring for your [ELDER]. Choose only one answer for each statement from the following: A great deal [4], Quite a lot [3], Somewhat [2], A little [1], or Not at all [0].

$\begin{array}{ccccc}\begin{array}{c}\text { Great } \\ \text { deal }\end{array} & \begin{array}{l}\text { Quite } \\ \text { a lot }\end{array} & \begin{array}{l}\text { Some } \\ \text { what }\end{array} & \begin{array}{c}\text { A } \\ \text { little }\end{array} & \begin{array}{l}\text { Not at } \\ \text { all }\end{array}\end{array}$

1. I feel God will bless me.

4

3

2

2. I feel better about myself.

$4 \quad 3$

3

$4 \quad 3$

3. I feel I have become a stronger tolerant, and/or patient person around persons with sickness or handicaps.

4. I feel having others say that 4 taking care of my relative is the right thing to do is important.

5. I feel that my relative will 4 my care remember me in his/her will for care

6. I feel someone will

take care of me when I need it.

7. I feel nurses, doctors, 4 and social workers work harder to care for my

[ELDER] too.

8. I feel that placing

3

2

2

2

2

0

my [ELDER] in

a nursing home will be avoided.

7. I feel that doctors, 4 3 2 nurses and social workers do not know everything about my [ELDER]'s chances for getting better. 
Now I'd like to talk to you about some of the ways people feel about caring for another person. Please tell me how you feel now about caring for your [ELDER]. Choose only one answer for each statement from the following: A great deal [4], Quite a lot [3], Somewhat [2], A little [1], or Not at all [0].

$\begin{array}{ccccc}\text { Great } & \text { Quite } & \text { Some } & \text { A } & \text { Not at } \\ \text { deal } & \text { a lot } & \text { what } & \text { little } & \text { all }\end{array}$

10. I feel receiving a

smile,touch,or

eye contact

from my [ELDER]

is important.

11. I feel I have a closer

relationship with

my [ELDER].

12. I feel I have an

opportunity to

repay my [ELDER]

for a past debt.

13. I feel receiving a

"thank you"

14. I feel I have

become a better person by

learning new

information

15. I feel I have

become a better.

person by learning

new ways to care

for the elderly.
4

3

4

3

2

1

0

4

3

2

1

0

4

3

2

1

0

4

3

2

1

0

4

3

2

1

0 
Now I'd like to talk to you about some of the ways people feel about caring for another person. Please tell me how you feel now about caring for your [ELDER]. Choose only one answer for each statement from the following: A great deal [4], Quite a lot [3], Somewhat [2], A little [1], or Not at all [0].

$\begin{array}{ccccc}\text { Great } & \text { Quite } & \text { Some } & \text { A } & \text { Not at } \\ \text { deal } & \text { a lot } & \text { what } & \text { little } & \text { all }\end{array}$

16. I feel that I have made many new friends.
4

3 what little all

$\begin{array}{llllll}\begin{array}{l}\text { 17. I feel more important. } \\ \text { 18. I feel I have }\end{array} & 4 & 2 & 1 & 0 \\ \text { the freedom to } & 4 & 3 & 2 & 1 & 0\end{array}$

make decisions that matter.

19. I feel I do not need

to hold a job.

20. I feel that receiving praise and admiration for my efforts from doctors, nurses and social workers is important

21. I feel I can now plan my own schedule each day.

22. I feel happier now than I did before I started caring for my [ELDER].
4

3

3

3

3
2

1

0

0

0

0

0

0 
Now I'd like to talk to you about some of the ways people feel about caring for another person. Please tell me how you feel now about caring for your [ELDER]. Choose only one answer for each statement from the following: A great deal [4], Quite a lot [3], Somewhat [2], A little [1], or Not at all [0].

$\begin{array}{ccccc}\text { Great } & \text { Quite } & \text { Some } & \text { A } & \text { Not at } \\ \text { deal } & \text { a lot } & \text { what } & \text { little } & \text { all }\end{array}$

\footnotetext{
23. I feel that caring for my [ELDER] has made our family grow and work closer together.
}

24. I feel my family members now look up to me because of my efforts under difficult circumstances.

\section{[IF CARE RECEIVER LIVES WITH RESPONDENT:]}

$\begin{aligned} & 25 . \text { I feel having my } \\ & \text { relative live with }\end{aligned}$
me means added
money coming
into the house.

Source. Picot, S. (1995). Rewards, costs and coping of African American caregivers. Nursing Research, 44(3), 147-152.Permission granted by Sandra J. Fulton Picot 
Appendix H

Institutional Review Board Approval Letter 


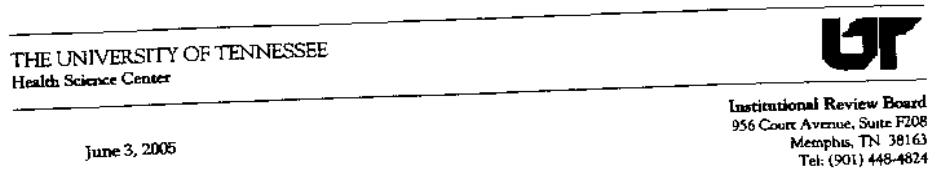

Lois Bolden, APRN, BC

c/o Mona Wicks, PhD.

College of Graduate Coll

Department of Nursing

UTHSC

CAMPUS

Dear Ms. Bolden.

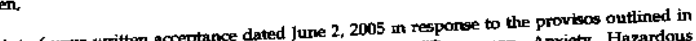
We are in receipt of your written acceptance dated june 2, 2005 in "Depression, Anciety, Hazardous

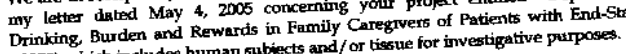

\#8270) which ixcludes humar subjects and/or tige

The Aderion The Adninistratve Section of the Uned to be consistent with the guidelines for expediemplying with proper consideration of the rights and appication was approved in this regard as cod, and the potential benefits of the study. This letirs welfare of humann subjects, the risk in lication; questionnaire(s); brochure; fyers; posters and consent constitules fill approva or yol jated June 3, 2005 and stamper approved by the

form(s) [Patient and Caregiver, dated 12 montths with an expiration date of May 4, 2006. The
2005 . This project is approved for 12006 .

form(s) dated June 3, 2005 are also approved from the drtion In the event that volunteers, either subjects or patients, are to be sect such solicitation maberiabs (ie., and standard patient care practices,

to and approved by the UTHSC Any alberations (revisions) in the pratocoi nuust be promptiy reported to and approved by thing to the IRB prior to imiplementation of these revisnons. You have nidividual resposts.

Board in the event of urantucipated or serions adverse events and sibje In addition, reapproval of your project is required by the Irr, and is regardless of the time the activity has

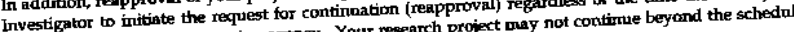
been approved by the sponsming agency. Your research projtime the project.

as indicated above unless you obtain wr

Simerely,
Done-nee
Terrence F. Ackerman, Ph.D.
Exectitive Vice Chairman
Institutional Review Board 
Appendix I

\section{Caregiver Informed Consent Form}




\section{CAREGIVER INFORMED CONSENT FORM}

Title of Research Study:

Principle Investigator:

Co-Investigators:
Depression, Anxiety, Hazardous Drinking, Burden and Rewards in Family Caregivers of Patients with End-Stage Liver Disease

Lois Bolden, APRN, BC, Ph.D. candidate University of Tennessee Health Science Center College of Nursing 663 Lamar Alexander Building 877 Madison Avenue Memphis, TN 38163

Mona Newsome Wicks, Ph.D., RN Caroline Riely, M.D.

\section{INTRODUCTION}

You have been given the opportunity to participate in a research study that will describe depressive symptom levels, anxiety levels, frequency of hazardous drinking, rewards, and subjective burden reported by family caregivers of patients with chronic liver disease. Another purpose of the study is to determine the predictors of subjective burden and mental and physical health status of African American and Caucasian family caregivers of persons with chronic liver disease. You understand that to participate in this study, you will be one of about 73 family caregivers invited to participate in this study from the practice of Dr. Riely, Dr. Fleckenstein and Dr. Sachdev. The estimated time for each subject to complete the study is 30-45 minutes. The duration of the entire study is 1 year.

\section{PROCEDURES TO BE FOLLOWED}

You will be asked to complete study questionnaires because you were identified as the primary caregiver of the person that receives care in this clinic. You understand that completing the questionnaires will take about 30 to 45 minutes of your time. The study and its procedures have been approved by appropriate faculty members and review board at the University of Tennessee at Memphis. If you are selected to participate in this study, the researcher will ask you to complete: 1) a sociodemographic (seeking information related to age, education and relationship to the care recipient) and several mental health questionnaires (measuring depressive and anxiety symptoms and patterns of alcohol use) 2) questionnaires related to your caregiving experience (stress and rewards related to caregiving experience). There are no physical, psychological, social, or economical risks associated with this study.

You understand that there are three (3) ways that you can participate: 
1. The first way that you can participate in the study is that you can be recruited in the clinic by the researcher. After an explanation of the study, if you choose to participate, you will read and sign a consent form and keep a copy for yourself. You can complete the questionnaires in an area of your choice (within the clinic) and return the questionnaires to the researcher. After the questionnaires have been completed and returned, the researcher will review your responses for completeness. If there are incomplete items on the questionnaire the researcher will obtain the responses restating there were no right or wrong answers. You will be given a $\$ 20.00$ Walmart gift certificate at the time that the completed questionnaires are returned to the researcher.

2. The second way that you can participate in the study is by taking the questionnaires home to complete them. If you choose to you can carry the packet home to complete the questionnaires, you understand that the packet will include two copies of the informed consent, a letter explaining the study, questionnaires, and the researcher's business card including a cell phone number for any questions about the study. If you choose to participate, you will read and sign the consent form and complete the questionnaires. You understand that the researcher will provide an envelope with return postage and you will receive a follow up telephone call from the researcher within 24 hours after receipt of the questionnaires. The purpose of this call will be to answer any questions you may have regarding the completion of the questionnaire packet. After the questionnaires have been completed and returned, the researcher will review your responses. If there are incomplete items on the questionnaire, the researcher will telephone you within 48 hours to obtain your responses restating there are no right or wrong responses to the questions. If the consent forms are not signed the researcher will call you within 24 hours and send another informed consent within 48 hours for you to sign and mail back to the researcher in an envelope with return postage. If the questionnaires are not returned within 5 days, another packet will be mailed to you to complete. A $\$ 20.00$ Walmart gift certificate will be mailed to you within one week after return of completed questionnaires by mail.

3. The third way that you can participate in this study is if your family member who has liver disease brings the study packet home to you. If the packet is brought to you by your family member, you understand that the packet will include two copies of the informed consent, a letter explaining the study, questionnaires, and the researcher's business card including a cell phone number for any questions about the study. If you choose to participate, you will read and sign the consent form and complete the questionnaires. You understand that the researcher will provide an envelope with return postage and you will receive a follow up telephone call from the researcher within 24 hours after receipt of the questionnaires. The purpose of this call will be to answer any questions you may have about the completion of the questionnaires. If the consent forms are not signed, the researcher will call you within 24 hours and send another informed 
consent within 48 hours for you to sign and mail back in an envelope with return postage. If the questionnaires are not returned within 5 days, another packet will be mailed to you to complete. After the questionnaires have been completed and returned, the researcher will review the responses. If there are incomplete items on the questionnaire, the researcher will telephone you within 48 hours to obtain my responses, reiterating that there were no wrong or right answers on the questionnaire items. You will be mailed a \$20.00 Walmart gift certificate within 1 week when the completed questionnaires are returned to the researcher. A summary of the results for the entire sample can be mailed to your home address if you request it after the completion of the study.

\section{RISK ASSOCIATED with PARTICIPATION}

You understand that there is minimal risk associated with participation in this study. However, completing the questionnaires may provoke emotional feelings and recall of painful experiences for the caregiver. Another risk involves being identified as someone with significant psychosocial problems. You will be provided contact telephone numbers in the event you later desire to communicate any concerns or need additional information.

\section{BENEFITS ASSOCIATED WITH PARTICIPATION:}

One benefit of participation in this study is that you will be screened for and told if you have significant depressive symptoms, anxiety, or hazardous drinking behaviors. If your scores suggest that you may have significant depressive symptoms and/or anxiety or hazardous dinking behaviors, you will be asked to sign a no harm contract and receive a referral letter to share with your health care provider. Information from this study will provide the healthcare providers who care for patients with liver disease information about the caregiving experience. This information will also provide other healthcare professionals with information that they can use to determine how to better provide healthcare and assistance to family caregivers.

\section{ALTERNATIVES TO PARTICIPATION:}

Your family member (the person with liver disease) will receive the same care from the liver doctor's office whether or not you choose to participate in this study. If you choose not to participate in the study, then you will not have to complete the informed consent or complete the questionnaires.

\section{CONFIDENTIALITY:}

You understand that any information obtained about you during your participation in this study, including questionnaires will be confidential. However, you understand that representatives of the University of Tennessee Health Science Center Institutional Review Board, College of Nursing, and the practice of Dr. Riely, Dr. Fleckenstein and 
Dr. Sachdev will have access to confidential data that identify you by name. You understand that third parties such as insurers or employers will not have access to the research data.

You also understand that to insure confidentiality and anonymity, your information will be kept in a completed packet. Each packet will be assigned a code number. A master list that links names and code numbers will be kept in a separate and secure location. All documents will be maintained in a secure file cabinet with access restricted to the investigators in the College of Nursing, at the University of Tennessee Health Science Center for a period of no longer than 7 years. Individual subjects will not be identified in any presentations or publication of the results of the data. The information published or presented from this study will refer to the groups in this study.

Under federal privacy regulations, you have the right to determine who has access to your personal health information (called "protected health information" or PHI). Protected health information collected in this study will include basic demographic information such as your age, gender employment status, your personal and family history of depression, drinking, and anxiety. By signing this consent form, you are authorizing Lois Bolden, the researcher, at The University of Tennessee to have access to your protected health information collected in this study and ask questions pertaining to protected health information from you.

In addition, your PHI may be shared with other persons involved in the conduct or oversight of this research, including faculty at the University of Tennessee Health Science Center. The Institutional Review Board (IRB) at the University of Tennessee Health Science Center may review your PHI as part of its responsibility to protect the rights and welfare of research subjects. Your PHI will not be used or disclosed to any other person or entity, except as required by law or for authorized oversight of this research study by other regulatory agencies or for other research for which the use and disclosure of your PHI has been approved by the IRB. Your PHI will be used only for the research purposes described in the introduction of this consent form and your PHI will be used until the study is completed.

You may cancel this authorization in writing at any time by contacting the principal investigator listed on the first page of the consent form. If you cancel the authorization, continued use of your PHI is permitted if it was obtained before the cancellation and its use is necessary in completing the research. However, PHI collected after your cancellation may not be used in the study. If you refuse to provide this authorization, you will not be able to participate in the research study. If you cancel the authorization, then you will be withdrawn from the study. Finally, federal regulations allow you to obtain access to your PHI collected or used in this study.

\section{COMPENSATION AND TREATMENT FOR INJURY:}

I understand that I am not waiving any legal rights or releasing the University of Tennessee or its agents from liability for negligence. I understand that, in the event of physical injury resulting from research a procedure, the University of Tennessee has no funds budgeted for compensation either for lost wages or for medical treatment. 
Therefore, the University of Tennessee does not provide for treatment or reimbursement for such injuries.

\section{QUESTIONS:}

The study has been explained to you in detail and you have had ample opportunity to have your questions answered. If you have any other questions now or at any time during the research study that are not answered during your clinic visits, you may contact the researcher at the following telephone numbers with any questions or concerns you may have. You may contact the researcher to report research related injuries such as worsening depression or any condition that might be associated with completing the questionnaires. You may call collect at any time, 24 hours a day, and 7 days a week at 901-448-6178. You may also call Dr. Mona Newsome Wicks, Ms. Bolden's advisor at University of Tennessee, Memphis College of Nursing at 901-448-6125. You may contact Dr. Clair E. Cox, UTHSC IRB chairman at (901) 448-4824 if you have any questions about your rights as a participant in this study or your rights as a research subject.

\section{PAYMENT FOR PARTICIPATION:}

You will receive a Wal-Mart Gift Card valued at twenty-dollars (\$20) as a token of appreciation for participating in this study.

\section{COST OF PARTICIPATION:}

You understand that by choosing to participate in this research study, under no circumstances will you incur monetary or other charges for questionnaires completed or postage used as part of this study's protocol. All funds required for the study will be provided by the investigator.

\section{VOLUNTARY PARTICIPATION:}

Since you are the caregiver of the person who is a patient at this clinic, you have been approached as a potential volunteer participant. You understand that your participation in this study is totally voluntary and at anytime you may withdraw from or refuse to participate in this study. Therefore, if you choose not to participate in the study, your family member will still receive the same care as usual. You have been given the opportunity to ask any questions about the study or about being a participant. Further, you know that you may call the researcher at any time with any questions at (901) 4486157 (the researcher's office) or (901)517-6139 (the researcher's cell phone). If you decide to withdraw from this study, you will not be penalized and no loss will be incurred on you. 


\section{CONSENT OF SUBJECT:}

I have read, or have had read to me, the description of the research study as outlined above. The investigator or his/her representative has explained the study to me and has answered all of the questions I have at this time. I have been told of the potential risks, discomforts, side effects and adverse reactions as well as the possible benefits (if any) of the study.

I freely volunteer to participate in the study. I understand that I do not have to take part in this study and that my refusal to participate will involve no penalty or loss of rights to which I am entitled. I further understand that I am free to later withdraw my consent and discontinue participation in this study at any time. I understand that refusing to participate or later withdrawing from the study will not adversely affect my family member (the person with liver disease) subsequent medical care. I will receive a copy of this consent form.

Signature of Research Subject

Date

Signature of Legally Authorized Representative

Date

Relationship of Legally Authorized Representative

Date

Signature of Person conducting consent

Date

Signature of Witness

Date

Signature of Principal Investigator

Date 
Appendix J

Patient Informed Consent Form 


\section{PATIENT INFORMED CONSENT FORM}

Title of Research Study:

Principle Investigator:

Co-Investigators:
Depression, Anxiety, Hazardous Drinking, Burden and Rewards in Family Caregivers of Patients with End-Stage Liver Disease

Lois Bolden, APRN, BC, Ph.D. candidate University of Tennessee Health Science Center College of Nursing 663 Lamar Alexander Building 877 Madison Avenue Memphis, TN 38163

Mona Newsome Wicks, Ph.D., RN Caroline Riely, M.D.

\section{INTRODUCTION}

You are being given the opportunity to participate in a research study that will describe depressive symptom levels, anxiety levels, frequency, rewards and subjective burden reported by family caregivers of patients with chronic liver disease. Another purpose of the study is to determine the factors that predict subjective burden and mental and physical health status of African American and Caucasian family caregivers of persons with end stage liver disease.

You understand that you will be one of about 73 individuals with chronic liver disease who will be asked to participate in the study. Patients who are invited to participate in this study receive care from Dr. Riely, Dr. Fleckenstein, and Dr. Sachdev. The estimated time for each subject to complete the study is $30-45$ minutes. The duration of the entire study is 1 year.

\section{PROCEDURES TO BE FOLLOWED}

You will be asked to sign an informed consent form allowing the researcher to review your medical records to screen your bilirubin, albumin, and INR levels and the level of encephalopathy and ascites that you have. This information will be used to determine your Child-Pugh Score (CPS). The CPS is a measure of the severity of your liver disease. You understand that if your CPS is an A, B, or C that you meet the study criteria and will be asked to identify your family caregiver (the person who takes care of you or would take care of you if you could no longer care for yourself). You understand that it will take about 10-15 minutes of your time to have the purpose of the study explained to you and to complete the informed consent. The consent interviews will take place in an area of your choice (within the clinic)-If you are identified as a potential participant by CPS and are willing to participate in this study, you will read and sign a 
consent form and keep a copy for yourself. The study and its procedures have been approved by appropriate faculty members and review board at the University of Tennessee at Memphis. If your family caregiver is selected to participate in this study, the researcher will explain (1) the purpose of the study and (2) informed consent to you. The researcher will ask your family caregiver to complete 1) a sociodemographic questionnaire, 2) several mental health questionnaires, and 2) questionnaires related to the caregiving experience. You are not expected to complete these questionnaires. You understand that there are no physical, psychological, social, or economical risks associated with participating in this study.

Once the consent form is signed and returned to the researcher, you understand that there are three ways your family caregiver can participate.

4. If your family caregiver is present during your clinic appointment the researcher will begin the interview with your family caregiver after an explanation of the study has been given and informed consent is signed. Your family caregiver can complete and return the questionnaires to the researcher during the clinic visit. After the questionnaires have been completed and returned, the researcher will review your caregivers' responses. If there are incomplete items on the questionnaire, the researcher will obtain the responses restating that there are no right or wrong answers. Your family caregiver will be given a \$20.00 Wal-Mart gift certificate at the time that the completed questionnaires are returned to the PI.

5. If your caregiver chooses to complete the questionnaires at home, you understand that the researcher will provide an envelope with return postage. Your caregiver will receive a follow-up telephone call from the researcher within 24 hours after receipt of the questionnaires. The purpose of this call will be to answer any questions my caregiver may have regarding the completion of the questionnaire packet. After the questionnaires have been completed and returned, the researcher will review the caregivers' responses for completeness. If there are incomplete items on the questionnaire, the PI will telephone the caregiver within 48 hours to obtain their responses to the missing items, restating there was no right or wrong responses to the questions. If the consent forms are not signed the researcher will call the caregiver within 24 hours and send another informed consent within 48 hours for your caregiver to sign and mail back to the researcher in an envelope with return postage. If your caregiver has not returned the questionnaires within 5 days another packet will be mailed to the caregiver. A \$20.00 Wal-Mart gift certificate will be mailed to your caregiver within one week after return of completed questionnaires by mail.

6. You understand that if you meet the criteria for inclusion in the study and are not accompanied by your caregiver during the clinic visit that the researcher will give you a questionnaire packet to give to your caregiver. The packet will include two copies of the informed consent, a letter explaining the study, questionnaires, and the researchers' business card including a cell phone number for any questions pertaining to the study. The researcher will also provide an envelope with return postage so your caregiver can return the questionnaire packet to the researcher via the US mail. A follow-up call within 24 hours will occur to answer questions that your caregiver may have about the study. After the questionnaires have been 
completed and returned, the researcher will review the caregivers' responses. If there are incomplete items on the questionnaire, the researcher will telephone the caregiver within 48 hours to obtain their responses, restating that there were no wrong or right answers to the questionnaire items. If the consent forms are not signed, the researcher will call your family caregiver within 24 hours and send another informed consent to sign within 48 hours and for your family caregiver to mail back to the researcher in an envelope with return postage. If the caregiver has not returned the questionnaires within 5 days another packet will be mailed to the caregiver. A $\$ 20.00$ Wal-Mart gift certificate will be mailed to your caregiver within one week after return of completed questionnaires by mail.

A summary of the results for the entire sample can be mailed to your home address if you request it after the completion of the study.

\section{RISK ASSOCIATED WITH PARTICIPATION}

You understand that there is no risk associated with participation in this study. I will be provided with contact telephone numbers in the event you later desire to communicate any concerns or need additional information.

\section{BENEFITS ASSOCIATED WITH PARTICIPATION:}

You will not directly benefit from participation in the study. However, information from this study will help health care providers find new ways to improve the lives of families and consequently patients coping with end stage liver disease. By helping with this study, information related to the caregiving experience will also provide other healthcare professionals with information that they can use to determine how to better provide healthcare and assistance to future patients with liver disease and their caregivers.

\section{ALTERNATIVES TO PARTICIPATION:}

You will receive the same care from the liver doctor's office whether or not you choose to participate in this study. If you choose not to participate in the study, then you will not have to complete the informed consent and information will not be obtained from your medical record.

\section{CONFIDENTIALITY:}

You understand that any information obtained about you during your participation in this study, including your CPS will be confidential. However, you understand that representatives of the University of Tennessee Health Science Center Institutional Review Board, College of Nursing and the practice of Dr. Riely, Fleckenstein, and Sachdev will have access to confidential data that identify you by name. You understand that third parties such as insurers or employers will not have access to the research data.

You also understand that to insure confidentiality and anonymity, your information and your caregiver's information will be kept in a completed packet. Each 
packet will be assigned a code number. A master list that links names and code numbers will be kept in a separate and secure location. All documents will be maintained in a secure file cabinet with access restricted to the investigators in the College of Nursing, at the University of Tennessee Health Science Center for a period of no longer than 7 years. Individual subjects will not be identified in any presentations or publication of the results of the data. The information published or presented from this study will refer to the groups in this study.

Under federal privacy regulations, you have the right to determine who has access to your personal health information (called "protected health information" or PHI). PHI collected in this study will include the components of my CPS i.e. bilirubin, albumin, INR, encephalopathy and ascites rating. By signing this consent form, you are authorizing Lois Bolden, the researcher, at the University of Tennessee to have access to your PHI collected in this study and to receive your PHI from the facility where you have received health care. In addition, your PHI may be shared with other persons involved in the conduct or oversight of this research, including faculty at the University of Tennessee Health Science Center. The Institutional Review Board (IRB) at the University of Tennessee Health Science Center may review your PHI as part of its responsibility to protect the rights and welfare of research subjects. Your PHI will not be used or disclosed to any other person or entity, except as required by law or for authorized oversight of this research study by other regulatory agencies or for other research for which the use and disclosure of your PHI has been approved by the IRB. Your PHI will be used only for the research purposes described in the introduction of this consent form and your PHI will be used until the study is completed.

You may cancel this authorization in writing at any time by contacting the principal investigator listed on the first page of the consent form. If you cancel the authorization, continued use of your PHI is permitted if it was obtained before the cancellation and its use is necessary in completing the research. However, PHI collected after your cancellation may not be used in the study. If you refuse to provide this authorization, you will not be able to participate in the research study. If you cancel the authorization, then you will be withdrawn from the study. Finally, federal regulations allow you to obtain access to your PHI collected or used in this study.

\section{COMPENSATION AND TREATMENT FOR INJURY:}

I understand that I am not waiving any legal rights or releasing the University of Tennessee or its agents from liability for negligence. I understand that, in the event of physical injury resulting from research a procedure, the University of Tennessee does not have funds budgeted for compensation either for lost wages or for medical treatment. Therefore, the University of Tennessee does not provide for treatment or reimbursement for such injuries. I or any insurance carrier will be billed for the costs associated with the medical treatment of a research related injury. 


\section{QUESTIONS:}

The study has been explained to you in detail and you have had ample opportunity to have your questions answered. If you have any other questions now or at any time during the research study that are not answered during your clinic visits, you may contact the PI at the following telephone numbers with any questions or concerns you may have. You may call collect at any time, 24 hours a day, and 7 days a week at 901-448-6157. You may also call Dr. Mona Wicks, Ms. Bolden's advisor at the University College of Tennessee at 901-448-6125. You may contact Dr. Clair E. Cox, UTHSC, IRB Chairman at 901-448-4824 if you have any questions about your rights as a participant in this study.

\section{PAYMENT FOR PARTICIPATION:}

You understand that you will receive no payment for participation in this study.

\section{COST OF PARTICIPATION:}

You understand that by choosing to participate in this research study, under no circumstances will you incur monetary or other charges for questionnaires completed or postage used as part of this study protocol. All funds for required for study will be provided by the investigator.

\section{VOLUNTARY PARTICIPATION:}

Since you are already a patient at this clinic, you have been approached as a potential volunteer participant. You understand that your participation in this study is totally voluntary and at anytime you may withdraw from or refuse to participate in this study. Therefore, if you choose not to participate in the study, you will receive the same care as usual from the clinic. You have been given the opportunity to ask any questions about the study or about being a participant. Further, you know that you may call the researcher at any time with any questions at 901-448-6157 (her office telephone number) or 901-517-6139 (her cell phone). If you decide to withdraw from this study, you will not be penalized and no loss will be incurred on you.

\section{CONSENT OF SUBJECT:}

I have read, or have had read to me, the description of the research study as outlined above. The investigator or his/her representative has explained the study to me and has answered all of the questions I have at this time. I have been told of the potential risks, discomforts, side effects and adverse reactions as well as the possible benefits (if any) of the study. I freely volunteer to participate in the study. I understand that I do not have to take part in this study and that my refusal to participate will involve no penalty or loss of rights to which I am entitled. I further understand that I am free to later withdraw my consent and discontinue participation in this study at any time. I understand that 
refusing to participate or later withdrawing from the study will not adversely affect my subsequent medical care. I will receive a copy of this consent form.

Signature of Research Subject

Signature of Legally Authorized Representative

Relationship of Legally Authorized Representative

Signature of Person Conducting Consent

Signature of Witness

Signature of Principal Investigator
Date

Date

Date

Date

Date

Date 


\section{VITA}

Lois Vennesta Bolden was born in Piperton, Tennessee, on August 14, 1953. She was awarded an Associate Degree and a Bachelor of Science in Nursing from The University of Memphis in Memphis, Tennessee, in 1976 and 1989, respectively. She graduated from University of Central Arkansas in Conway, Arkansas, in 1993 with a Master of Science in Nursing. She has over 25 years of experience as a nurse. She was employed in several roles, such as staff nurse, nursing supervisor, director of nursing, nursing research manager, and psychiatric clinical specialist.

She was certified as a Clinical Specialist in Adult Psychiatric and Mental Health Nursing by ANCC in 1997. She was a recipient of the Sigma Theta Tau scholarship and Ethnic Minority Pre-doctoral Fellowship from Substance Abuse Mental Health Services Administration (SAMHSA). She has published several manuscripts as a primary and secondary author in the following journals: Journal of Nursing Care Quality, American Journal of Nursing, The Journal of Advanced Nursing and Clinical Nursing Research, Issues in Mental Health. She has presented at several national conferences.

Lois Bolden received a Doctor of Philosophy in nursing degree from The University of Tennessee Health Science Center in December, 2006. Her dissertation research focused on Mental Health Status, Subjective Burden, and Caregiver Rewards in Family Caregivers of Patients with Chronic Liver Disease. Presently she is employed by The University of Tennessee Health Science Center as an Instructor in the undergraduate program. 